\title{
On stability in matching and strategy-proofness in social choice
}

Citation for published version (APA):

Khare, S. (2019). On stability in matching and strategy-proofness in social choice. [Doctoral Thesis, Maastricht University]. ProefschriftMaken Maastricht. https://doi.org/10.26481/dis.20190315sk

Document status and date:

Published: 01/01/2019

DOI:

10.26481/dis.20190315sk

Document Version:

Publisher's PDF, also known as Version of record

\section{Please check the document version of this publication:}

- A submitted manuscript is the version of the article upon submission and before peer-review. There can be important differences between the submitted version and the official published version of record.

People interested in the research are advised to contact the author for the final version of the publication, or visit the DOI to the publisher's website.

- The final author version and the galley proof are versions of the publication after peer review.

- The final published version features the final layout of the paper including the volume, issue and page numbers.

Link to publication

\footnotetext{
General rights rights.

- You may freely distribute the URL identifying the publication in the public portal. please follow below link for the End User Agreement:

www.umlib.nl/taverne-license

Take down policy

If you believe that this document breaches copyright please contact us at:

repository@maastrichtuniversity.nl

providing details and we will investigate your claim.
}

Copyright and moral rights for the publications made accessible in the public portal are retained by the authors and/or other copyright owners and it is a condition of accessing publications that users recognise and abide by the legal requirements associated with these

- Users may download and print one copy of any publication from the public portal for the purpose of private study or research.

- You may not further distribute the material or use it for any profit-making activity or commercial gain

If the publication is distributed under the terms of Article $25 \mathrm{fa}$ of the Dutch Copyright Act, indicated by the "Taverne" license above, 


\section{On Stability in Matching and Strategy-Proofness in Social Choice}

Netherlands, 2019

Written by

\section{SHASHWAT KHARE}

Maastricht University 
(c) Shashwat Khare, Maastricht 2019.

All rights reserved. No part of this publication may be reproduced, stored in a retrieval system, or transmitted in any form, or by any means, electronic, mechanical, photocopying, recording or otherwise, without the permission from the author.

This book was typeset by the author using $\mathrm{HT}_{\mathrm{E}} \mathrm{X}$.

Published by ProefschriftMaken || www.proefschriftmaken.nl ISBN: 978-94-6380-261-1

Printed in The Netherlands by ProefschriftMaken || www.proefschriftmaken.nl 


\title{
On Stability in Matching and Strategy-Proofness in Social Choice
}

\author{
DISSERTATION \\ to obtain the degree of Doctor at the Maastricht University, \\ on the authority of the Rector Magnificus, \\ Prof.dr. Rianne M. Letschert \\ in accordance with the decision of the Board of Deans, \\ to be defended in public \\ on Friday $15^{\text {th }}$ March 2019 at 12:00 hours
}

by

Shashwat Khare 


\section{Supervisor}

Prof. dr. H.J.M. Peters

\section{Co-supervisor}

Dr. A.J.A. Storcken

\section{Assessment Committee}

Prof. dr. D. Vermeulen (Chairman)

Dr. A. Mackenzie

Prof. dr. B. Klaus (University of Lausanne, Switzerland)

Prof. dr. A. Westkamp (University of Cologne, Germany)

Dit onderzoek werd financieel mogelijk gemaakt door de Graduate School of Business and Economics (GSBE). 
To my family, for their undying love and support. 



\section{Acknowledgements}

Having completed my Bachelor in Statistics, I started my Master is Statistics at the same university in July 2013. I was not fully convinced then whether I wanted to devote my future to the development of scientific research. However, not content with my approach during the Bachelor's, I decided to devote more time trying to go into the depths of the subjects whilst also trying to acquire knowledge about other topics which were not part of the course work. It was during this time my fondness for Quantitative Economics increased, making me take that particular specialisation in the second year of my Master's degree. I had the privilege of working under the tutelage of great scientists. It was then, that I got the chance to undertake my very first scientific research study under the guidance of Dr. Priyodorshi Banerjee. That study made me realise that I wanted to dedicate more time in research with the hope of exploring new topics and finding new results.

However, the main credit for my decision to come to Maastricht in pursuit of my $\mathrm{PhD}$ has to go to Dr. Souvik Roy. It was during his courses when I realised how much I was fascinated by the concepts of Game Theory, Mechanism Design and Social Choice Theory among others. He helped me understand the key concepts of the subjects, imbibing in me a great curiosity for all these topics. Moreover, being a part of Maastricht University himself, our association continued even during my $\mathrm{PhD}$, where we worked on several projects together. 
My years as a $\mathrm{PhD}$ researcher in Maastricht have been one of the most significant years in my life. I truly had a wonderful time here, learning a lot along the way. This time wouldn't have been the same if not for the people who were always there to guide me, help me and spend time with me during thick and thin.

First and foremost, I would like to express my deepest gratitude to my supervisors, Prof. Dr. Hans Peters and Dr. Ton Storcken. Throughout my stay in Maastricht they have not only been the guiding pillars of my $\mathrm{PhD}$, but have also been great friends. I thank them for their insightful comments on my research. They were always there to help me out with great ideas whenever I felt stuck in my thesis. Our weekly meetings were something I always used to look forward to. Not only did we discuss great ideas for my research, but many a time we also ended up having conversations on music, culture etc. once our academic discussions got over.

Next, I would like extend a word of thanks to my colleagues from the Quantitative Economics Department. Yolanda and Karin, we are truly thankful to have the two of you. You were always there to help me with all little things related to the department, teaching and what not. Having those small conversations with you guys was always a treat. Fellow and former $\mathrm{PhD}$ candidates, thank you for all the great times we had. I enjoyed every bit of it. I would like to thank a few of you in particular. First off, Jan, my first office mate: thank you for helping me settle in along with Marc. Anna, thank you for helping me out with my presentations and your enthusiasm which always motivated me and cheered me up. Abhinaba Da and Swarnendu Da, our Indian dinners, trips and conferences were always fun. The joint meetings we had with our supervisors were always great and so were those breaks we used to take to have hot chocolate. Alex, it was always fun to watch football games with you. Aida, I will miss how we always spent time joking around and pulling each other's legs. Tim, Veerle and Niels, thank you for being my Dutch translators. I know I bothered you a lot of times with the letters I got in Dutch. Thank you for being patient with me and always helping me out. Benoit, thank you for all the board game nights you organised. Etiënne, your useful tips were truly and highly helpful. Adi, thank you for sharing your office with me. It was also nice to finally find a colleague with whom I could discuss DC-Marvel on top of Game Theory and Social Choice Theory. Roland, my 'special-beer-tasting' buddy: the conversations we had with Adi on time travel and multiverse on numerous occasions were the best. Thank you for sharing the wonderful journey on 'untappd' with me. Sean, thank you for introducing me to the great football club of Maastricht and being a great football teammate along with an amazing colleague. The way we destroyed others during our beerpong matches surely tops it all. Thank you for being there when the going got tough. This list would be incomplete without a shout out to Dewi, Luca and all my colleagues with 
whom I took lunch and coffee breaks. It was great to have after-work drinks and dinner with you guys from time to time. I could still mention so many of you, but please let me write this: thank you all from the bottom of my heart. This journey would have been impossible without all of you. This goes for PhDs from the other departments as well.

I would also like to take this opportunity to thank my football club DBSV red socks. The time I spent training for football made sure that I could always divert my mind and get back to my research with a greater determination. Even though my time in the club was marred with injuries, I would always cherish the time I spent there. I would like to thank all my teammates of indoor as well as outdoor teams, especially Cayle, Rick, Ronan, Jan and Jacob. I would also like to thank my coaches and the friends I made there. The 'DBSV activites' were always great and refreshing.

Deciding to leave India and come here for my research was indeed not an easy choice. I am so thankful to all the people who helped me settle in. Swapnil Dada and Megha Didi, thank you for helping me settle in when I was missing India in my initial days. I am also truly blessed to have friends who became my family away from home. Especially everyone from my college who decided to come to Netherlands or nearby to do their respective PhD. Arkajyoti Da and Basundhara Di, thank you for hosting me and Lalu almost every other weekend. Rudra Da, Tamal Da, Rajarshi Da, Moy Da, Tom Da, Avi Da, Sunil Da, Anushri Di, Saagnik Da and Saswati Di, even though I am not a fan of getting up at 6 and going to hike, I still always looked forward to all the meetings that we had simply because of all the love and affection I got when I was with you all.

The last year of my $\mathrm{PhD}$ was especially tough. I would like to thank everyone who helped me in every way possible during this time. It was your help that allowed me to get over the line and successfully complete my PhD. I would not have been able to do it without you. This not only includes my family, supervisors, colleagues, football teammates and college friends, but also some new friends I made then. Ilona and Tuomas, thank you for starting the bridge sessions along with Benoit, Adi and me. And thank you Li, for joining our sessions after Tuomas left. Stuti, thank you for sharing my interest in horror movies. It was always fun to meet you, along with Jaspreet, Adi, Raghav, Chinmay, Ujjwala and Atul to cook nice Indian dinner and just relax. Eileen, thank you for teaching me some German and some really cool games like Looping Louie.

Finally, I would like to thank my family for everything they have done for me. For their love, support and care. Bhaiyasaheb and Pappa, I am sure you would have been 
really happy and proud to see me finish my $\mathrm{PhD}$. Tai and Mamma, thank you for being a constant source of inspiration and constantly showering love upon me. Aai, Baba, Kaka and Kaku, I am what I am because of you. Thank you for helping me through my tough times, cheering me up when I was down and always inspiring me. Thank you my 'Bhawandas' (including my sister in laws and Vedaant). The fact that I could call you and share my problems at any hour of the day gave me a lot of confidence and helped me focus better. Finally, I would like to thank every member of the 'TaiBhaiyasaheb' group. Our family bonding gave me the strength and willpower to push harder and overcome all the difficulties I faced during my time here. I am grateful to all of you.

Shashwat Khare

Maastricht, March 2019 
Contents

Contents

1 Introduction 1

1.1 Stability in Matching with Couples having Non Responsive Preferences 5

1.2 Stability in Matching with Couples having Responsive Preferences . . 5

1.3 Collective Choice Rules on Convex Restricted Domains . . . . . . . . 6

I Matching Theory 9

2 Stability in Matching with Couples having Non-Responsive Preferences 11 
2.1 Introduction . . . . . . . . . . . . . . . 11

2.2 The framework ....................... . . 14

2.2.1 Matching ..................... 14

2.2.2 Preferences ..................... 15

2.2 .3 Stability........................... 18

2.2.4 Two well-known algorithms . . . . . . . . . . . 20

2.3 Stability is not guaranteed under RVT . . . . . . . . . . . . . 21

2.4 Existence of stable matching when couples' preferences satisfy RVT . 23

2.5 Existence of stable matching with strong CPI . . . . . . . . . . 27

2.6 Matching market with non-identical hospital preferences . . . . . . . 34

2.7 Conclusion ......................... 41

3 Stability in Matching with Couples having Responsive Preferences 43

3.1 Introduction . . . . . . . . . . . . . . 43

3.2 The framework ...................... 46

3.2 .1 Matching ................... 46

3.2.2 Preferences ...................... 47

3.2.3 Stability ........................ 49

3.2 .4 Algorithm ........................ 51

3.3 Stable matching is not guaranteed at arbitrary responsive profiles . . . 54

3.4 Conditions on couples' preferences for stability . . . . . . . . . . 57

3.5 Conditions on hospitals' preferences for stability . . . . . . . . . . 60 
3.6 Concluding remarks . . . . . . . . . . . . . . . 64

II Social Choice Theory $\quad 65$

4 Collective Choice Rules on Convex Restricted Domains 67

4.1 Introduction . . . . . . . . . . . . . . . . 67

4.2 Convex sets of preferences . . . . . . . . . . . . 71

4.3 Convex restricted domains $\ldots \ldots \ldots 72$

4.4 Possibilities under Unanimity . . . . . . . . . . . . . . . . . . 74

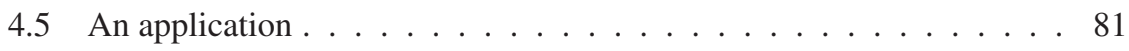

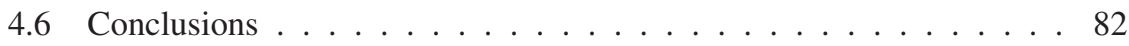

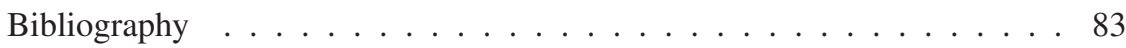

$\begin{array}{lr}\text { Bibliography } & 85\end{array}$

$\begin{array}{lr}\text { Valorisation } & \mathbf{8 9}\end{array}$

$\begin{array}{ll}\text { Curriculum Vitae } & 91\end{array}$ 

List of Tables

2.1 No Stable Matching under RVT . . . . . . . . . . . . 22

2.2 Non-Identical Hospital Preferences . . . . . . . . . . . . . . . . . 35

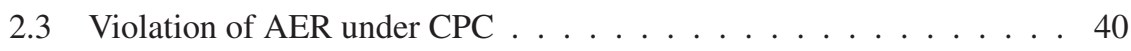

2.4 Violation of CPC under AER . . . . . . . . . . . . . . . 40

3.1 No Stable Matching under Responsive Preferences (Ex. 1) . . . . . . 54

3.2 No Stable Matching under Responsive Preferences (Ex. 2) . . . . . . 56

3.3 Stability with Unemployment Aversion . . . . . . . . . . . . 63 



\section{CHAPTER 1}

Introduction

This thesis is divided in two parts. The first part contains Chapters 2 and 3 which deal with matching theory, and the second part consists of Chapter 4 which deals with social choice theory.

Matching is a mapping from elements of one set to the elements of another set where the elements of each pair mutually benefit from each other. Matching Theory, as the name suggests deals with the concepts and frameworks of matching. To understand more about matching, consider an example where individuals need to be assigned to institutions. This is an example of many-to-one matching, because an individual can only be matched to one institution, whereas an institution may be related to more individuals than one. This includes a variety of scenarios, for example admitting students to colleges or schools, employing workers to firms, assigning doctors to hospitals etc. For the sake of convenience, we consider the problem of assigning doctors to hospitals. Naturally, we would like this matching to satisfy some 'nice' properties. One such property is that a doctor should prefer her assigned hospital to being unemployed and a hospital should prefer a doctor assigned to it rather than having 
a vacancy for that position. It is easy to see that if a matching does not satisfy this property, then a doctor will prefer to leave the hospital she is assigned to and rather be unemployed than being at this hospital. Similarly, a hospital will want to remove such an unwanted doctor that is assigned to it and would rather like to have a position vacant than have such a doctor. To understand the second property, consider a situation where a doctor $d_{1}$ is matched to hospital $h_{1}$. But $d_{1}$ prefers another hospital $h_{2}$ over $h_{1}$ and $h_{2}$ prefers $d_{1}$ over some doctor matched to it. Thus, $\left\{d_{1}, h_{2}\right\}$ would jointly 'block' this matching. Therefore, the second property we would like a matching to satisfy is that it should be immune to any such blocks. A matching which satisfies these two properties is called stable. Since a hospital can be matched with more than one doctor, it needs to have a preference over sets of doctors. Thus, if a hospital has a preference over individual doctors, it needs to extend this preference over sets of doctors. In the literature responsiveness is a very well studied extension of hospitals' preferences on individuals in order to obtain a preference over the sets of doctors. Responsiveness means that if a hospital compares two sets of doctors which differ in exactly one doctor, then the hospital would prefer the set with the more preferred doctor. For example, a hospital will prefer the set of doctors $\left\{d_{1}, d_{2}\right\}$ to $\left\{d_{1}, d_{3}\right\}$, if and only if the hospital prefers $d_{2}$ to $d_{3}$.

The classical result of Gale and Shapley (1962) implies that it is possible to solve the problem of stable matching when individuals need to be matched with institutions when institutions have responsive preferences. Their famous 'deferred acceptance algorithm' shows exactly how to form such a stable matching for this problem. A problem arises when there is a presence of couples among doctors. This problem stems from the fact that the members of a couple might no longer behave like separate individual doctors, but rather have a joint preference over pairs of hospitals. Like hospitals' preferences, responsiveness of couples' preferences is also a widely studied concept. A couple's preference satisfies responsiveness if unilateral improvement in the position of one member of the couple is better for the couple itself. Thus, if one member of the couple is fixed at hospital $h_{1}$, then the couple will prefer a matching where the other member is matched to $h_{2}$ over $h_{3}$, if and only if that member prefers $h_{2}$ to $h_{3}$. However, responsiveness does not determine a complete set of preferences for hospitals or couples. This is because all sets of doctors or all pairs of hospitals cannot be compared using responsiveness. For example, consider four doctors $d_{1}, d_{2}, d_{3}, d_{4}$, and suppose a hospital $h$ prefers $d_{i}$ to $d_{i+1}$ for all $i \in\{1,2,3\}$. Responsiveness does not give an ordering between $\left\{d_{1}, d_{4}\right\}$ and $\left\{d_{2}, d_{3}\right\}$ for the hospital. This is because $d_{1}$ is the most preferred doctor among all the doctors, but $d_{4}$ is the least preferred one. It is easy to see that the same phenomenon also follows while considering responsive couples' preferences. 
Given a preference of hospitals on individual doctors, we can have many preferences on sets of doctors satisfying responsiveness. One such preference is when a hospital replicates this preference on individual doctors in order to obtain a preference on sets of doctors. What this means is that if some hospital $h_{1}$ has a vacancy of ten doctors, it is the same as having ten identical hospitals with one vacancy each, such that each hospital has the same preference on individual doctors as $h_{1}$. What this in turn implies is that, if a couple tries to block with a hospital, then the hospital would only consider one member at a time for one position, instead of considering both members together for two positions. Consider the above example where we have four doctors and a hospital $h$. Moreover, let $\left\{d_{1}, d_{4}\right\}$ be a couple in the above example and let $h$ be matched to $\left\{d_{2}, d_{3}\right\}$. As noted above, responsiveness does not specify an ordering between $\left\{d_{1}, d_{4}\right\}$ and $\left\{d_{2}, d_{3}\right\}$. Thus, if the couple tries to apply to the hospital, then instead of comparing the set of doctors $\left\{d_{1}, d_{4}\right\}$ to $\left\{d_{2}, d_{3}\right\}$, the hospital makes comparisons individually. Thus, since $d_{1}$ is better but $d_{4}$ is worse, the hospital rejects this proposal. Klaus and Klijn (2005), showed that if the preferences of hospitals are responsive but replicated then we have a stable matching for any responsive preferences of the couples. Two questions naturally arise from this. One is, what happens in this set-up if couples' preferences violate responsiveness. And the second one is, to check whether the same result holds even when we assume that hospitals can have any responsive preferences instead of just having replicated responsive preferences. We try to answer the first question in Chapter 2 where we assume that couples violate responsiveness only if they can be together at the same hospital and we try to answer the second question in Chapter 3.

As the name suggests, Social Choice Theory deals with the techniques for finding a collective choice for society from the given set of alternatives depending on the preferences of the individuals. The set of alternatives may concern a great variety of things, for example candidates to vote in an election, a location for a public good, etc. However, a rational individual would want the best possible choice outcome according to her preference. Since the individual preferences are private information, this might create an incentive for someone to strategically misreport her true preference. For example, consider three individuals $1,2,3$ and three alternatives $a, b, c$. If two or more individuals have the same top ranked alternative, then let the rule choose that alternative and in case all the individuals have different tops, let the rule choose alternative $a$. Now suppose the top ranked alternatives for 1,2,3 are $a, b, c$ respectively. But suppose 2 prefers $c$ to $a$. Thus if all of them report their true preferences, $a$ will be chosen but if 2 misreports her true preference where $c$ is her top ranked alternative, then $c$ is chosen which she prefers to $a$. In order to avoid this manipulation, we expect our social choice rule to satisfy a property called strategy-proofness. If a choice rule 
satisfies strategy-proofness, then an individual cannot be better of by manipulating her true preference. Thus this property ensures that individuals honestly report their true preferences. Another property that a choice rule is usually assumed to satisfy is that of unanimity, where if an alternative is the top ranked alternative for all the individuals, then it is chosen. Although these are the most common properties which a choice function is assumed to follow, we can have other such properties depending on different contexts.

Gibbard (1973) and Satterthwaite (1975) famously showed that if we have at least three alternatives and if we allow for the individuals to have any possible preference then the only rule to satisfy the above mentioned nice properties is a dictatorial one. This leads to a natural question, whether we can have non-dictatorial choice rules satisfying the nice properties by enforcing some restrictions on our problem. A natural way to do so is by restricting the domain of preferences. One such very well known restriction is that of single-peaked preferences, where each agent has a best ranked alternative (peak) and the outcomes further from this alternative are preferred less compared to alternatives that are nearer to the peak. To see that this domain does not contain all possible preferences, let $a, b, c$ be three alternatives placed sequentially on a horizontal axis. It is clear that $a c b$ and $c a b$ are not examples of single-peaked preferences. It is because if $a$ is the peak, then $b$ is closer to $a$ than $c$, thus $c$ can never be preferred to $b$. There have been many other restrictions of domains that have been studied in literature.

We assume such a domain restriction in Chapter 4. We assume that every individual has a partial ordering, and that the domain of preferences of this individual includes all the preferences containing this partial ordering. It appears that this domain of preferences is convex with respect to Kemeny distance. As shown in Kemeny et. al. [1962], the Kemeny distance between two sets is the symmetric set difference between them. Thus, given any two preferences the Kemeny distance counts the pairs that are differently ordered in these two preferences. Therefore, given any two preferences $P_{1}$ and $P_{2}$, the Kemeny distance between these two preferences is the minimum number of switches of adjacent alternatives required to reach one preference from another, i.e., it gives us the shortest path to reach from one preference to another. For example, consider three alternatives $a, b, c$ and consider two preference profiles $a b c$ and $c a b$. From $a b c$, we first need to switch $b$ and $c$ to get the preference $a c b$, followed by a switch between $c$ and $a$ to get preference $c a b$. Therefore, the Kemeny distance between these two preferences is two. The preference $a c b$ is called the intermediate preference between the preference $P_{1}$ and $P_{2}$. A set of preferences $V$ is called convex if for any two preferences $P_{1}$ and $P_{2}$ in $V$ and any preference $P_{3}$, if $P_{3}$ is an intermediate preference of $P_{1}$ and $P_{2}$, then $P_{3}$ lies in 
$V$.

Now, we will give a short description of the work we have done in the subsequent chapters.

\subsection{Stability in Matching with Couples having Non Responsive Preferences}

In chapter 2 we consider many-to-one matching between hospitals and doctors. However, the matching market contains some couples who view the pair of jobs as complements. We allow for couples' preferences to violate responsiveness only if they can be together at the same hospital. The preferences of the hospitals are assumed to be responsive. We assume that these preferences over sets of doctors are replications of preferences over individual doctors. To begin with, we assume that the hospitals have a common preference over doctors, however, their preference over sets of doctors may vary. This applies to situations when there is a common admission test leading to a common ranking. We characterise all preferences of couples where a stable matchings exists. We find that this only happens when the lower ranked member of the couple is ready to 'compromise' for the higher ranked member. That is, the couples' preferences satisfy responsiveness with respect to the higher ranked member of the couple. Then we study the condition on the preferences of hospitals which allow for a stable matching in case couples' preferences are unrestricted. Finally, we relax the assumption of common preference on individual doctors by the hospitals and establish a sufficient condition for the existence of a stable matching.

\subsection{Stability in Matching with Couples having Re- sponsive Preferences}

In chapter 3 as in 2, we study many-to-one matching where there are couples among doctors. However, the couples' as well as hospitals' preferences satisfy responsiveness. Contrary to Chapter 2, we assume that different hospitals can have different preferences over the individuals. What differs in Chapter 3 is that hospitals can have any responsive preference over the sets of doctors. Thus when a couple applies to 
the same hospital together, the hospital considers them for two positions simultaneously, as opposed to Chapter 2, where the members of the couple are considered one position at a time. We find that even though couples' preferences satisfy responsiveness, stability is not guaranteed. The result obtained here is a remarkable because responsiveness appears to be a natural extension of individual preferences where we expect to find stability. We then try to find restrictions on the preferences of couples, which guarantee the existence of a stable matching for any responsive preferences of hospitals. Finally, we look at restrictions on the preferences of the hospitals, which are necessary and sufficient for the existence of stable matching for any responsive preferences of couples.

\subsection{Collective Choice Rules on Convex Restricted Do- mains}

In chapter 4, we study the sets of preferences that are convex with respect to the Kemeny distance of preferences. It appears that the convex sets consist of all preferences containing a certain partial ordering and the other way around all preferences containing a given partial ordering form a convex set. We then consider restricted domains where each individual has a partial ordering and the domain of preferences of this individual contain all preferences containing this partial ordering. What is interesting here is that different individuals can have different domains of preferences depending on the partial ordering which need not be the same for everybody. We call this partial ordering - a priori information as this partial ordering is already known in advance for every individual. But even though this a priori information is known for every individual, the preference of an individual over the whole set of alternatives is still private information. Necessary and sufficient conditions are then formulated under which a restricted domain admits unanimous, strategy-proof and non-dictatorial choice rules. Naturally, this work can be applied to a variety of problems, because we often come across different individuals having different a priori information. And even though the complete preference of an individual might not be known, a partial ordering of that individual might still be public information. This can happen due to various restrictions which can thus lead to entirely different domains of preferences for them. For example, consider a situation where some friends need to decide on a common dish to eat for everybody. One of the friends is vegetarian and will prefer any dish over a dish with meat. Another friend likes meat and will prefer to have meat over any other dish. Even though this a priori information is common knowledge, still the entire preferences of these two friends is private information. However, 
the difference in a priori information will lead to a completely different domain of preferences for these two friends, as the preference of these friends over vegetarian dishes and dishes with meat is exactly opposite. 



\section{Part I}

\section{Matching Theory}



CHAPTER 2

\section{Stability in Matching with Couples having Non-Responsive Preferences}

\subsection{Introduction}

In many different contexts, there is a centralized matching procedure by which individuals on one side of the market are matched to institutions on the other side of the market. These include the market for lawyers in Canada, children in schools in the USA, doctors and senior-level health-care professionals in several countries, etc. A matching is pairwise stable if there does not exist any institution-individual pair that can block it by getting matched together, such that both of them are better off compared to their original matching.

Roth (1984)[25] showed that it is possible to design mechanisms which incentivise only one side of the market to truthfully reveal their preferences. However, the results on stability have been more promising. The received doctrine is that stable matchings 
do exist under appropriate domain restrictions. In particular, institutions have to view individuals as substitutes and individuals must care only about institutions to which they are matched.

It was first pointed out by Roth (1984)[26] that the presence of couples in the labour market may lead to an impossibility result where no stable matching exists. This can happen because couples may view pairs of jobs as complements. In other words, the assumption that choices of individuals are independent of each other might not apply. Klaus and Klijn (2005) showed that a stable matching exists when couples' preferences satisfy responsiveness, meaning that a couple is better off when any member of the couple is matched to a more preferred institution keeping the other member fixed. However, Kojima, Pathak and Roth (2010) pointed out that responsiveness is not satisfied in their data set because couples show strong preference to be matched to institutions situated in the same geographical area.

As we have discussed above, matching models disregarding responsive couples preferences are very common. However, taking such preferences into account may come at the cost of losing stability. We feel that this general non-existence cannot be the final conclusion. This is primarily because, even though there are profiles where stable matchings do not exist, it might be very well true that such profiles are rare. For instance when the disregard for responsiveness occurs in the irrelevant part of the preferences, i.e. the parts that will never be reached by a matching procedure. Moreover, stability is a property of a matching at a given preference profile, and in many practical scenarios, there are natural restrictions on profiles. Therefore, it is important to know whether at a given profile, a stable matching exists. Here we present a first step in that. We show that there is a problem and moreover formulate a sufficient condition for couples preferences when there is no further knowledge on the other agents in the matching procedure.

As in Chapter 1, we consider a matching problem between a set of hospitals and a set of doctors including some couples. We focus on the issue of existence of stable matchings with couples. We first look at the scenario when all hospitals have a common preference over individual doctors. This can be easily justified if hospitals rank doctors according to their grades of some common examination. Hospitals' preferences over sets of doctors are derived from the common preference by using responsiveness. Responsiveness means that for two allocations of a hospital, which differ by exactly one doctor, the hospital prefers the allocation with the better doctor. However, these preferences might vary from one hospital to the other. Each individual doctor has a strict preference over hospitals. The preference of a couple is derived from the preferences of members of the couple. In view of the preceding 
discussion, we let couples' preferences violate responsiveness in an appropriate way to capture their willingness to be matched together. Thus, in the spirit of Dutta and Massó (1997), we assume that a couple prefers to be matched at the same hospital rather than being matched to different hospitals. We show that if hospitals have a common preference over doctors, then stable matching exists if and only if each couple's preference does not violate responsiveness with respect to the more preferred member of the couple (according to the common preference of hospitals).

Next, we consider the scenario where a couple is allowed to have arbitrary nonresponsive preferences over pairs of hospitals. In other words, a couple need not always prefer to stay together, they may like to stay apart as well. We show that when couples' preferences are arbitrary, then stable matching exists if and only if for each couple one of the following happens: (i) either the members of the couple are ranked consecutively or (ii) there is at most one doctor ranked in-between the members and one member of the couple is ranked at the bottom of the common preference of hospitals.

Finally, we relax the assumption of a common preference of hospitals. First, we show by means of an example that if the relative ordering of members of a couple is different in different hospitals and that couples' preferences violate responsiveness in order to be together, then a stable matching may not exist. In view of this, we assume the relative ordering of members of each couple to be the same in every hospital's preference and provide a sufficient condition for the existence of a stable matching.

This chapter is organised as follows. We formally introduce the model, providing all the necessary definitions, notations and algorithms which are used throughout this chapter in Section 2.2. We provide an example in Section 2.3 to show that stability is not guaranteed when couples violate responsivness to be together even when hospitals have common preference on the individuals. In Section 2.4, we provide a necessary and sufficient condition on couples' preferences for the existence of a stable matching when institutions have a common preference over individual doctors. Section 2.5 deals with further restrictions on the common preference of hospitals and establishes conditions on the same that guarantee existence of a stable matching when couples' preferences are unrestricted. Finally, in Section 2.6, we relax the assumption of a common preference of hospitals and provide sufficient condition for existence of stable matching. 


\subsection{The framework}

We consider many-to-one matching ${ }^{1}$ between doctors and hospitals. We denote by $H$ the set of hospitals. We use the notation $\bar{H}$ to denote $H \cup\{\emptyset\}$. The interpretation of $\emptyset$ is that if some doctor is matched to $\emptyset$, then that doctor is practically unmatched (i.e., is not assigned to any hospital). Each hospital $h \in H$ has a finite capacity, denoted by $\kappa_{h} \geq 2$.

We denote by $D$ the set of doctors. We assume that $D=F \cup M \cup S$, where $F, M, S$ are pairwise disjoint sets of doctors. We denote the doctors in $F$ by $\left\{f_{1}, \ldots, f_{k}\right\}$ and those in $M$ by $\left\{m_{1}, \ldots, m_{k}\right\}$, for some $k \in \mathbb{N}$ where $\mathbb{N}$ denotes the set of natural numbers. This, in particular, means that $F$ and $M$ have the same number of doctors. The doctors in $F$ and $M$ together form fixed couples, whereas the doctors in $S$ are not part of any couple. We call the doctors in $S$ single doctors and those in $M$ or $F$ non-single doctors. We denote the set of couples by $C=\left\{\left\{f_{1}, m_{1}\right\}, \ldots,\left\{f_{k}, m_{k}\right\}\right\}$ and a generic couple by $c=\{f, m\}$.

Throughout this chapter, we assume $|H| \geq 2,|D| \geq 4$ and $|C| \geq 1$. That is, there are at least two hospitals and four doctors including at least one couple. We also assume that the total number of vacancies in all hospitals in $H$ is equal to the total number of doctors available, i.e., $\sum_{h \in H} \kappa_{h}=|D|$.

An allocation of a couple $c=\{f, m\}$ is an element $\left(h, h^{\prime}\right)$ of $\bar{H}^{2}$ where hospitals $h$ and $h^{\prime}$ are matched with doctors $f$ and $m$, respectively. As we have already mentioned, here one or both of $h$ and $h^{\prime}$ might be empty, which would mean that the corresponding doctor(s) is(are) not matched with any hospital.

For notational convenience, we do not use braces for singleton sets.

\subsubsection{Matching}

A matching is an allocation of the doctors over the hospitals such that the total allocation of doctors in a hospital does not exceed its capacity and a doctor is allocated to at most one hospital (i.e., is allocated to exactly one hospital or no hospital). Below, we provide a formal definition of this.

\footnotetext{
${ }^{1}$ See Sönmez(1996), and Martínez et al.(2000) for some work on stability in many-to-one matching.
} 
Definition 2.1. A matching on $H \cup D$ is a mapping $\mu$ on $H \cup D$ such that:

(i) for all $h \in H, \mu(h) \subseteq D$ with $|\mu(h)| \leq \kappa_{h}$,

(ii) for all $d \in D, \mu(d) \in \bar{H}$,

(iii) for all $d \in D$ and all $h \in H, \mu(d)=h$ if and only if $d \in \mu(h)$.

\subsubsection{Preferences}

In this section, we introduce the notion of preferences of doctors and hospitals. We also propose certain restrictions on those.

For a set $X$, we denote by $\mathbb{L}(X)$ the set of linear orders, i.e., complete, reflexive, transitive, and antisymmetric binary relations over $X$. An element $R \in \mathbb{L}(X)$ is called a preference over $X$ and $P$ is the strict part of $R$. Since a preference is antisymmetric, $x R y$ implies either $x=y$ or $x P y$. For $P \in \mathbb{L}(X)$ and $k \leq|X|$, we define $r_{k}(P)$ as the $k$-th ranked alternative in $P$, that is, $r_{k}(P)=x$ if and only if $|\{y \in X: y R x\}|=k$. Moreover, for $P \in \mathbb{L}(X)$ and $x \in X$, we define by $r(x, P)$ the rank of $x$ in $P$, that is, $r(x, P)=k$ if and only if $r_{k}(P)=x$.

\section{Preferences of Hospitals}

For any hospital $h \in H$, a preference of $h$ over individual doctors, denoted by $\tilde{P}_{h}$, is defined as an element of $\mathbb{L}(D \cup\{\emptyset\})$.

We assume $d \tilde{P}_{h} \emptyset$ for all $d \in D$ and all $h \in H$. That is, a hospital always prefers to have a doctor than having a vacant position.

For a hospital $h$, the feasible sets of doctors (given its capacity) is defined as $\left\{D^{\prime} \subseteq D\right.$ : $\left.\left|D^{\prime}\right| \leq \kappa_{h}\right\}$. A preference over feasible sets of doctors of a hospital $h$ is an element of $\mathbb{L}\left(\left\{D^{\prime} \subseteq D:\left|D^{\prime}\right| \leq \kappa_{h}\right\}\right)$. In what follows we discuss how a preference of a hospital over individual doctors is extended to that over feasible sets of doctors. We introduce the notion of responsiveness in this context.

Responsiveness captures the idea of separability that is used in the context of extending preferences over individual dimensions to that over over multi-dimensions. Roughly speaking, responsiveness says that hospitals always prefer it when they get 
a better doctor (or set of doctors). For example, consider a preference $\tilde{P}_{h}$ of a hospital over the individual doctors $\left\{d_{1}, d_{2}, d_{3}, d_{4}\right\}$, where $d_{1} \tilde{P}_{h} d_{2} \tilde{P}_{h} d_{3} \tilde{P}_{h} \tilde{P}_{h} d_{4}$. Then, responsiveness says that the pair $\left(d_{1}, d_{2}\right)$ will be preferred to the pair $\left(d_{1}, d_{3}\right)$, the set $\left(d_{1}, d_{2}, d_{4}\right)$ will be preferred to the set $\left(d_{1}, d_{3}, d_{4}\right)$, etc. in the extension of $\tilde{P}_{h}$ over feasible sets of doctors. It is important to note that responsiveness does not say how the hospital $h$ will compare certain sets of doctors, for instance, the pairs $\left(d_{1}, d_{4}\right)$ and $\left(d_{2}, d_{3}\right)$. So, one can have a responsive extension of $\tilde{P}_{h}$ where the first pair is preferred to the second, and another where the second pair is preferred to the first. Below, we provide a formal definition of responsive extension.

Definition 2.2. Let $h$ be a hospital with capacity $\kappa_{h}$ and let $\tilde{P}_{h}$ be a preference of $h$ over individual doctors. Then, a preference $P_{h}$ of $h$ over feasible sets of doctors satisfies responsiveness with respect to $\tilde{P}_{h}$ if

(i) the restriction of $P_{h}$ over individual doctors coincides with $\tilde{P}_{h}$, that is, for all $d, d^{\prime} \in D \cup\{\emptyset\}, d P_{h} d^{\prime}$ if and only if $d \tilde{P}_{h} d^{\prime}$, and

(ii) for all $D^{\prime} \subsetneq D$ and all $D_{1}, D_{2} \subseteq D \backslash D^{\prime}$ such that $\left|D^{\prime} \cup D_{1}\right| \leq \kappa_{h}$ and $\left|D^{\prime} \cup D_{2}\right| \leq$ $\kappa_{h}$, we have $\left(D^{\prime} \cup D_{1}\right) P_{h}\left(D^{\prime} \cup D_{2}\right)$ if and only if $D_{1} P_{h} D_{2}$.

Next, we define the notion of common preference of hospitals over individual doctors. As the name suggests, this simply says that all the hospitals have the same preference over the individual doctors. Such a preference can be viewed as the common ranking of the individual doctors based on the grades of some common examination, etc. Note that hospitals may, in principle, differ on the extension of this common preference over sets of feasible doctors.

Definition 2.3. Let $\left\{P_{h}\right\}_{h \in H}$ be a collection of preferences of hospitals over feasible sets of doctors and let $P^{0} \in \mathbb{L}(D \cup\{\emptyset\})$. Then, $\left\{P_{h}\right\}_{h \in H}$ is said to satisfy Common Preference over Individual doctors (CPI) with respect to $P^{0}$ if for all $h \in H, P_{h}$ is responsive with respect to $P^{0}$.

Unless mentioned otherwise, we assume CPI for every collection of preferences of hospitals. Whenever we consider a collection of preferences satisfying CPI with respect to $P^{0}$, we assume for ease of presentation that the indexation of the doctors in couples is such that $f P^{0} m$ for every couple $c=\{f, m\} \in C$, and that of the couples in $C=\left\{\left\{f_{1}, m_{1}\right\}, \ldots,\left\{f_{k}, m_{k}\right\}\right\}$ is such that $m_{1} P^{0} m_{2} P^{0} \ldots P^{0} m_{k}$. This is without of loss of generality as we consider only one CPI at every given context. 


\section{Preferences of Doctors}

Every doctor has a preference over the hospitals including the 'empty' hospital $\emptyset$. Thus, a preference $P_{d}$ of a doctor $d \in D$ is an element of $\mathbb{L}(\bar{H})$. We assume $h P_{d} \emptyset$ for all $h \in H$ and all $d \in D$. In other words, all doctors prefer being matched to some hospital than being unemployed. Now, we proceed to define the preferences of couples based on the preferences of the members in it.

\section{Preferences of Couples}

Each couple has a preference over the pairs of hospitals. Thus, a preference $P_{c}$ of a couple $c$ is an element of $\mathbb{L}\left(\bar{H}^{2}\right)$. Recall that an allocation $\left(h_{1}, h_{2}\right)$ for a couple $c=\{f, m\}$ means that $f$ is matched with $h_{1}$ and $m$ is matched with $h_{2}$.

As we have discussed in Section 2.1, in this chapter we intend to deviate from responsiveness in a 'minimal' way and study its consequences on stability. We assume that a preference of a couple is responsive except in the situations where both the members of the couple get to stay together at some hospital. For instance, if $f$ prefers $h_{1}$ to $h_{2}$ and $m$ prefers $h_{2}$ to $h_{1}$, then, in contrast to responsiveness where the pair $\left(h_{1}, h_{2}\right)$ should have been preferred to both the pairs $\left(h_{1}, h_{1}\right)$ and $\left(h_{2}, h_{2}\right)$, we allow for the couple $\{f, m\}$ to prefer $\left(h_{1}, h_{1}\right)$ or $\left(h_{2}, h_{2}\right)$ or both to the pair $\left(h_{1}, h_{2}\right)$. Clearly, we allow this because at the allocation $\left(h_{1}, h_{1}\right)$ or $\left(h_{2}, h_{2}\right)$, the members of the couple can benefit from staying together. We call this 'preference for togetherness'. Note that we still assume that a couple prefers an allocation where both its members are matched to another where at least one member is unmatched.

To define the notion of responsiveness violated for togetherness, we use the notion of responsiveness for couples' preferences. This notion of responsiveness is exactly the same as that for a preference of a hospital. However, for the sake of completeness, we present the formal definition of this here.

Definition 2.4. Let $c=\{f, m\}$ be any couple and suppose $P_{f}$ and $P_{m}$ are the preferences of $f$ and $m$, respectively. Then, a preference $P_{c} \in \mathbb{L}\left(\bar{H}^{2}\right)$ of the couple $c$ is called responsive with respect to $P_{f}$ and $P_{m}$ if, for all $h, h_{1}, h_{2} \in \bar{H}$, we have

(i) $\left(h, h_{1}\right) P_{c}\left(h, h_{2}\right)$ if and only if $h_{1} P_{m} h_{2}$,

(ii) $\left(h_{1}, h\right) P_{c}\left(h_{2}, h\right)$ if and only if $h_{1} P_{f} h_{2}$, and

(iii) $\left(h_{1}, h_{2}\right) R_{c}(h,\{\emptyset\})$ and $\left(h_{1}, h_{2}\right) R_{c}(\{\emptyset\}, h)$. 
Now, we are ready to define the notion of responsiveness violated for togetherness for preferences of couples.

Definition 2.5. Let $c=\{f, m\}$ be any couple and let $P_{f}$ and $P_{m}$ be the preferences of $f$ and $m$, respectively. Then, a preference $\bar{P}_{c} \in \mathbb{L}\left(\bar{H}^{2}\right)$ of c satisfies responsiveness violated for togetherness (RVT) if there exists a responsive (with respect to $P_{f}$ and $P_{m}$ ) preference $P_{c}$ of $c$ such that

(i) for all $h \in H$ and all $\left(h_{1}, h_{2}\right) \in \bar{H}^{2}$, if $(h, h) P_{c}\left(h_{1}, h_{2}\right)$ then we have $(h, h) \bar{P}_{c}\left(h_{1}, h_{2}\right)$, and

(ii) for all $\left(h, h^{\prime}\right),\left(h_{1}, h_{2}\right) \in \bar{H}^{2}$ such that $h \neq h^{\prime}$ and $h_{1} \neq h_{2}$, we have $\left(h, h^{\prime}\right) P_{c}\left(h_{1}, h_{2}\right)$ if and only if $\left(h, h^{\prime}\right) \bar{P}_{c}\left(h_{1}, h_{2}\right)$.

Note that RVT implies that couples' preferences can violate responsiveness only in order to be together at some hospital. In other words, responsiveness can only violated in favor of the pairs of the form $(h, h)$. Also, by taking $h_{1}=h_{2}$ in Condition (i) of Definition 2.5, it follows that for all $h, h^{\prime} \in H,(h, h) P_{c}\left(h^{\prime}, h^{\prime}\right)$ if and only if $(h, h) \bar{P}_{c}\left(h^{\prime}, h^{\prime}\right)$.

\section{Preference profiles and matching problems}

A preference profile is a collection of preferences for all the doctors in $D$, all the couples in $C$, and all the hospitals in $H$, where hospitals' preferences are assumed to be responsive. Thus, a preference profile, denoted by $\underset{\sim}{P}$, is a collection of preferences $\left(\left\{{\underset{\sim}{P}}_{d}\right\}_{d \in D},\left\{{\underset{\sim}{P}}_{c}\right\}_{c \in C},\left\{{\underset{\sim}{P}}_{h}\right\}_{h \in H}\right)$ where for all $d \in D, c \in C$ and $h \in H,{\underset{\sim}{P}}_{d}$ is a preference of doctor $d,{\underset{\sim}{P}}_{c}$ is a preference of couple $c$ and $\underset{\sim}{P}$ is a responsive preference over feasible sets of doctors of hospital $h$, respectively.

By a matching problem, we mean a set of hospitals with corresponding capacities, a set of doctors with its partition into the sets $F, M$, and $S$, and a preference profile.

\subsubsection{Stability}

Our model is formally equivalent to a many-to-many matching market as a couple looks for two positions and hospitals have at least two positions. Thus, one can 
have different notions of stability based on different types of permissible blocking coalitions. $^{2}$

Blocking pairs can be a hospital and a single doctor or a pair of hospitals and a couple.

We say a hospital $h$ is 'interested' in a set of doctors $D^{\prime}$ at a matching $\mu$ if there is $D^{\prime \prime} \subseteq \mu(h)$ such that $\left\{\left(\mu(h) \backslash D^{\prime \prime}\right) \cup D^{\prime}\right\} P_{h} \mu(h)$. In other words, a hospital is interested in a set of doctors at a matching if it prefers to appoint those doctors by possibly removing some of its existing/matched doctors (to adjust its capacity). Similarly, we say a doctor $d$ (couple $c$ ) is interested in a hospital $h$ (pair of hospitals $\left(h, h^{\prime}\right)$ ) at a matching $\mu$ if $h P_{d} \mu(h)\left(\left(h, h^{\prime}\right) P_{c} \mu(c)\right)$. Note that if a hospital is interested in a set of doctors or a doctor (couple) is interested in a hospital (pair of hospitals) at a matching $\mu$, then it must be that they are not (already) matched at $\mu$.

Now, we define the notion of (individual) blocking between a hospital and a single doctor.

Definition 2.6. For a single doctor $s$, a hospital $h$, and a matching $\mu$, we say $(h, s)$ blocks $\mu$ if both $h$ and s are interested in each other at $\mu$.

Thus, a hospital and a single doctor block a matching if they are not matched together at that matching but prefer to be so.

Next, we define the notion of blocking between a pair of hospitals and a couple. A pair of hospitals and a couple, who are not already matched, blocks a matching if the couple prefers to be matched with that pair of hospitals, and the hospitals from that pair who are getting a new doctor from the couple is interested in getting it. Thus, the crucial thing here is that one of the members of the blocking couple might already be matched with one of the hospitals in the blocking pair. In that case, the other hospital must be interested in getting the other member of the couple. One might think that this case can be captured by our notion of (individual) blocking between the 'other hospital' and the 'other doctor'. Firstly, note that we have such notion of blocking only between hospitals and single doctors. Secondly, even if we define the notion of blocking between arbitrary (not necessarily single) individual doctor and hospital, that would not capture this situation as the other doctor might not be interested in the other hospital according to his/her individual preference but interested according to his/her couple preference.

\footnotetext{
${ }^{2}$ See Roth (1984) [7], Roth (1984) [8], Konishi and Ünver (2006), Echenique and Oviedo (2006), Hatfield and Kojima (2010), for some alternative notions of stability in many-to-many matchings.
} 
Definition 2.7. For a couple $c=\{f, m\}$, a pair of hospitals $\left(h_{f}, h_{m}\right)$, and a matching $\mu$, we say $\left(\left(h_{f}, h_{m}\right), c\right)$ blocks $\mu$ if $c$ is interested in $\left(h_{f}, h_{m}\right)$ at $\mu$, and

(i) if $\mu(x) \neq h_{x}$ for all $x \in\{f, m\}$, then $h_{f}$ is interested in $f$ and $h_{m}$ is interested in $m$,

(ii) if $h_{x}=\mu(x)$ and $h_{y} \neq \mu(y)$ for $x, y \in\{f, m\}$, then $h_{y}$ is interested in $y$.

One important thing to note about our notion of blocking is that here hospitals are myopic in the sense that when they remove a member of a couple, they do not consider the possibility of losing the other member of it. We consider this model as otherwise a farsighted notion of blocking would require a hospital to estimate the possibility of losing the 'other member' by checking whether there is really any hospital for that member to go (to be better off individually or by couple's preference). Such a notion will complicate the model considerably, and more importantly, doing so much of calculations in order to decide who to appoint or remove is practically impossible for big institutions like hospitals.

Definition 2.8. A matching $\mu$ is stable if it can not be blocked.

Remark 2.1. By our assumption that each hospital finds each doctor acceptable and each doctor finds each hospital acceptable, every stable matching is individually rational.

\subsubsection{Two well-known algorithms}

In this section, we present a well-known algorithm called doctor proposing deferred acceptance algorithm (DPDA). This algorithm was introduced by Gale and Shapley(1962). ${ }^{3}$ However, for our purpose, we modify this algorithm slightly. We use this modified algorithm to match hospitals with doctors. In what follows, we give a short description of DPDA, where each doctor $d$ has a preference $P_{d}$ over hospitals and each hospital $h$ has a preference $P_{h}$ over feasible sets of doctors.

DPDA: This algorithm has multiple stages. In stage 1, each doctor $d \in D$ proposes to his/her most preferred hospital according $P_{d}$. Each hospital $h \in H$ provisionally

\footnotetext{
${ }^{3}$ Since Gale and Shapley's work, stable matching problem in two sided matching has received a lot of attention (Knuth(1976), Gusfield and Irving(1989), Roth and Sotomayer(1990), Aldershof and Carducci(1996) being some notable examples of such work.
} 
accepts the most preferred collection of doctors according to $P_{h}$. If a hospital $h$ receives more than $\kappa_{h}$ proposals, then it keeps its most preferred $\kappa_{h}$ many doctors from these proposals and rejects all others. Having defined stages $1, \ldots, k$, the stage $k+1$ is defined in the following way: Each unmatched (till stage $k$ ) doctor $d$ proposes to his/her most preferred hospital from the remaining set of hospitals who have not rejected him/her in any of the earlier stages. If a hospital, whose provisional list of accepted doctors is less than its capacity, receives one or more fresh proposal, then it continues to add to its accepted list (till its capacity). However, if a hospital $h$, whose provisional list of doctors is equal to its capacity, receives one or more fresh proposal, then it accepts some of these fresh proposals by rejecting same number of relatively worse (according to $P_{h}$ ) doctors that it provisionally accepted earlier. The algorithm finally terminates when each doctor is either matched with some hospital or has been rejected by all hospitals.

Remark 2.2. In DPDA, each individual doctor proposes according to his/her individual preference. Therefore, couples do not play any role in it.

Now, we present another well-known algorithm called serial dictatorship algorithm (SDA). We give a short description of SDA where hospitals' preferences satisfy CPI with respect to $P^{0}$. Recall that unless otherwise mentioned, we assume that hospitals' preferences satisfy CPI property. That is, they have a common ranking, denoted by $P^{0}$, over individual doctors.

SDA: In SDA, the highest-ranked doctor according to $P^{0}$ chooses his/her mostpreferred hospital, and in general, the $j$-th ranked doctor according to $P^{0}$ chooses his/her most preferred hospital among the hospitals with available vacancy after all the better (with rank less than $j$ ) doctors have made their choices.

Our next remark is a standard result in matching theory.

Remark 2.3. Both DPDA and SDA produce the same matching when hospitals' preferences satisfy CPI.

\subsection{Stability is not guaranteed under RVT}

In this section, we explore the possibility of having a stable matching when couples' preferences satisfy RVT property, that is, are allowed to violate responsiveness in 
order for them to be employed together at a hospital. First, we show by the means of an example that, even when hospitals' preferences satisfy CPI, that is, have a common preference over individuals, a stable matching is not guaranteed in such situations.

Example 2.1. Suppose there are two hospitals each having capacity 2, two single doctors, and two other non-single doctors forming a couple. Formally, suppose $H=$ $\left\{h_{1}, h_{2}\right\}, \kappa_{h_{1}}=\kappa_{h_{2}}=2$, and $D=\left\{s_{1}, s_{2}, f, m\right\}$ where $c=\{f, m\}$ is the only couple. Consider the preference profile given in Table 2.1. Here, both the hospitals have a common preference over individual doctors which is denoted by $P^{0}$.

We do not present the preferences of hospitals over feasible sets of doctors because that does not play any role in this example. The couple's preference over pairs of hospitals, where one member is matched and the other one is unmatched, is not shown in the table, but is assumed to be responsive and ranked below the shown pairs.

\begin{tabular}{cccccc}
\hline$P^{0}$ & $P_{s_{1}}$ & $P_{s_{2}}$ & $P_{f}$ & $P_{m}$ & $P_{c}$ \\
\hline$f$ & $h_{2}$ & $h_{1}$ & $h_{2}$ & $h_{1}$ & $\left(h_{1}, h_{1}\right)$ \\
$s_{1}$ & $h_{1}$ & $h_{2}$ & $h_{1}$ & $h_{2}$ & $\left(h_{2}, h_{1}\right)$ \\
$s_{2}$ & & & & & $\left(h_{2}, h_{2}\right)$ \\
$m$ & & & & & $\left(h_{1}, h_{2}\right)$ \\
\hline
\end{tabular}

Table 2.1: No Stable Matching under RVT

Note that the couple's preference violates responsiveness in order to be together at $h_{1}$ since the pair $\left(h_{1}, h_{1}\right)$ is preferred to the pair $\left(h_{2}, h_{1}\right)$ in its preference.

Now we show that there does not exist a stable matching at the given preference profile. Suppose on the contrary that $\mu$ is a stable matching at this profile. Since the couple prefers to be matched to any pair of hospitals than having a member unmatched, it follows that both the members of the couple must be matched with some hospitals at the matching $\mu$. We consider all such allocations of the couple $c$, and show that blocking happens for each of these allocations.

(i) Suppose $\mu(c)=\left(h_{1}, h_{1}\right)$.

Since $h_{1} P_{s_{2}} h_{2}$ and $s_{2} P^{0} m,\left(h_{1}, s_{2}\right)$ blocks $\mu$.

(ii) Suppose $\mu(c)=\left(h_{2}, h_{1}\right)$.

Because $h_{2} P_{s_{1}} h_{1}$ and $s_{1} P^{0} s_{2} P^{0} m$, it must be that $\mu\left(s_{1}\right)=h_{2}$. Moreover, by responsiveness, $\{f, m\} P_{h_{1}}\left\{s_{2}, m\right\}$. This, together with the fact that $\left(h_{1}, h_{1}\right) P_{c}\left(h_{2}, h_{1}\right)$, implies $\left(\left(h_{1}, h_{1}\right), c\right)$ blocks $\mu$. 
(iii) Suppose $\mu(c)=\left(h_{2}, h_{2}\right)$.

Since $h_{2} P_{s_{1}} h_{1}$ and $s_{1} P^{0} m,\left(h_{2}, s_{1}\right)$ blocks $\mu$.

(iv) Suppose $\mu(c)=\left(h_{1}, h_{2}\right)$.

Because $h_{2} P_{s_{1}} h_{1}$ and $s_{1} P^{0} s_{2} P^{0} m$, we have $\mu\left(s_{1}\right)=h_{2}$. Moreover, by responsiveness $\{f, m\} P_{h_{2}}\left\{s_{1}, m\right\}$. This, together with the fact that $\left(h_{2}, h_{2}\right) P_{c}\left(h_{1}, h_{2}\right)$, implies $\left(\left(h_{2}, h_{2}\right), c\right)$ blocks $\mu$.

Since Cases (i)-(iv) are exhaustive, it follows that there does not exist a stable matching at the preference profile given in Table 2.1.

\subsection{Existence of stable matching when couples' pref- erences satisfy RVT}

In view of the fact that existence of stable matchings is not guaranteed when couples are allowed to violate responsiveness for togetherness, we search for additional conditions on couples' preferences so that the said existence is guaranteed.

Let $P_{C}^{0}=\left(\left\{P_{d}^{0}\right\}_{d \in D \backslash S},\left\{P_{c}^{0}\right\}_{c \in C}\right)$ be a given collection of preferences of non-single doctors (i.e., the doctors in $D \backslash S$ ) and couples such that for all $c \in C, P_{c}^{0}$ satisfies RVT. Given such a collection of preferences $P_{C}^{0}$, an extension of $P_{C}^{0}$ refers to any preference profile where (i) the preferences of non-single doctors and couples are as given in $P_{C}^{0}$, and (ii) hospitals' preferences satisfy CPI with respect to some preference $P^{0}$ over the individual doctors.

Recall that whenever hospitals' preferences over feasible sets of doctors satisfy CPI with respect to some preference $P^{0}$ over individual doctors, we assume that $f_{i} P^{0} m_{i}$ for each couple $\left\{f_{i}, m_{i}\right\}$.

In what follows, we present a condition, called responsive for $F$, that we use in describing situations where stable matchings exist. Responsive for $F$ property implies that couples' preferences are always responsive with respect to $f$. More precisely, if a couple moves together to a hospital from a pair of hospitals, then it must be that the $f$-member of the couple prefers that hospital to the hospital that he/she was originally matched with. In other words, compromise is always made by $m$ in order for a couple $\{f, m\}$ to be together at some hospital. 
Definition 2.9. A collection of preferences $P_{C}^{0}$ is said to be responsive for $F$ if for all $c=\{f, m\} \in C$ and all $h, h^{\prime} \in H,(h, h) P_{c}^{0}\left(h^{\prime}, h\right)$ implies $h P_{f}^{0} h^{\prime}$.

Now, we present our first theorem which provides a necessary and sufficient condition for the existence of stable matchings at every possible extension of a given collection of preferences $P_{C}^{0}$. In particular, it says that a stable matching exists at every possible extension of $P_{C}^{0}$ if and only if $P_{C}^{0}$ satisfies the responsive for $F$ property. In the interest of readability, we present the if part and the only if part of this theorem separately.

Theorem 2.1. (i) If $P_{C}^{0}$ satisfies responsive for $F$ property, then a stable matching exists at every extension of $P_{C}^{0}$.

(ii) If $P_{C}^{0}$ does not satisfy responsive for $F$ property, then there always exists an extension of $P_{C}^{0}$ at which there is no stable matching.

Proof. [Part (i)] The proof of this part is constructive. Suppose $P_{C}^{0}$ is responsive for F. We show that every extension $\underset{\sim}{P}$ of $P_{C}^{0}$ has a stable matching.

Take an extension $\underset{\sim}{P}$ of $P_{C}^{0}$. For all $m \in M$ and all $h \in H$, define the conditional preference of $m$ given $h$, denoted by $P_{m \mid h}^{0}$, as an element of $\mathbb{L}(H)$ such that $h^{\prime} P_{m \mid h}^{0} h^{\prime \prime}$ if and only if $\left(h, h^{\prime}\right) P_{c}^{0}\left(h, h^{\prime \prime}\right)$, where $c=\{f, m\}$.

Recall that by our initial assumption on CPI, $m_{i} P^{0} m_{j}$ for all $i, j \in\{1, \ldots, k\}$ such that $i<j$. In the following, we present an algorithm that produces a stable matching at $\underset{\sim}{P}$.

Algorithm 1: This algorithm involves $k+1$ steps. We present the $1^{\text {st }}$ step and a general step of the algorithm.

Step 1: Use SDA to match all the doctors ranked above $m_{1}$ according to $P^{0}$. Suppose $f_{1}$ is matched to some hospital $h_{1}$. Then, match $m_{1}$ using SDA where $m_{1}$ proposes according to the preference $P_{m_{1} \mid h_{1}}^{0}$.

Step j: Having matched all the doctors from the top till $m_{j-1}$ according to $P^{0}$ in steps 1 to $j-1$, use SDA to match all doctors ranked below $m_{j-1}$ and above $m_{j}$ according to $P^{0}$. Suppose $f_{j}$ is matched to some hospital $h_{j}$. Then, match $m_{j}$ by SDA where $m_{j}$ proposes according to the preference $P_{m_{j} \mid h_{j}}^{0}$. 
Continue this process till Step $k$ and then match the remaining single doctors by SDA in step $k+1$.

Let $\mu$ be the outcome of Algorithm 1. We show that $\mu$ is stable at $\underset{\sim}{P}$.

First, we show that $\mu$ cannot be blocked by $(h, s)$ for some $h \in H$ and $s \in S$. Assume for contradiction that some pair $(h, s)$ blocks $\mu$. By the nature of Algorithm 1, all doctors who propose before $s$ are ranked above $s$ in $P^{0}$. Since $s \notin \mu(h)$, this means either $\mu(s){\underset{\sim}{S}}_{s} h$ or $d P^{0} s$ for all $d \in \mu(h)$ and $|\mu(h)|=\kappa_{h}$. Clearly, if $\mu(s) \underset{\sim}{P_{s}} h$ then $s$ does not block with $h$. On the other hand, if $d P^{0} s$ for all $d \in \mu(h)$ and $|\mu(h)|=\kappa_{h}$, then by responsiveness of hospitals' preferences, we have $\mu(h) \underset{\sim}{P_{h}}((\mu(h) \backslash d) \cup s)$ for all $d \in \mu(h)$. Therefore, $h$ does not block with $s$. This proves that $\mu$ can not by blocked a hospital and a single doctor.

Now, we show that $\mu$ cannot be blocked by $\left(\left(h_{1}, h_{2}\right), c\right)$ for some $h_{1}, h_{2} \in H$ and $c \in C$. Assume for contradiction that some $\left(\left(h_{1}, h_{2}\right), c\right)$ blocks $\mu$. Let $c=\{f, m\}$. We complete the proof in two steps.

Step 1: In this step, we show that if $\left(\left(h_{1}, h_{2}\right), c\right)$ blocks $\mu$, then $\left(\left(\mu(f), h_{2}\right), c\right)$ also blocks $\mu$. Clearly, if $\mu(f)=h_{1}$, then there is nothing to show. So, suppose $\mu(f) \neq h_{1}$.

First, we claim $\mu(f) P_{f}^{0} h_{1}$. Assume for contradiction that $h_{1} P_{f}^{0} \mu(f)$. Since $f$ proposes according to $P_{f}^{0}$ and all the doctors that propose before $f$ are ranked above $f$ in $P^{0}$, $f \notin \mu\left(h_{1}\right)$ implies that $d P^{0} f$ for all $d \in \mu\left(h_{1}\right)$ and $\left|\mu\left(h_{1}\right)\right|=\kappa_{h_{1}}$. By responsiveness of hospitals' preferences, this means $\mu\left(h_{1}\right){\underset{\sim}{h_{1}}}_{h}\left(\left(\mu\left(h_{1}\right) \backslash d\right) \cup f\right)$ for all $d \in \mu\left(h_{1}\right)$. However, this contradicts that $\left(\left(h_{1}, h_{2}\right), c\right)$ blocks $\mu$. Therefore, $\mu(f) P_{f}^{0} h_{1}$.

Next, we show that $\left(\mu(f), h_{2}\right) P_{c}^{0}\left(h_{1}, h_{2}\right)$. Assume for contradiction that $\left(h_{1}, h_{2}\right) P_{c}^{0}\left(\mu(f), h_{2}\right)$. If $h_{1} \neq h_{2}$, then RVT implies $h_{1} P_{f}^{0} \mu(f)$, which is a contradiction. On the other hand, if $h_{1}=h_{2}$, then by the responsive for F property implies $h_{1} P_{f}^{0} \mu(f)$, which is a contradiction.

Now, we complete Step 1. Since $\left(\left(h_{1}, h_{2}\right), c\right)$ blocks $\mu$, it must be that $\left(\left(\mu\left(h_{2}\right) \backslash d\right) \cup m\right) P_{h_{2}} \mu\left(h_{2}\right)$ for some $d \in \mu\left(h_{2}\right)$. Because $\left(\mu(f), h_{2}\right) P_{c}^{0}\left(h_{1}, h_{2}\right)$, it follows that $\left(\left(\mu(f), h_{2}\right), c\right)$ blocks $\mu$.

Step 2: In this step, we show that $\left(\left(\mu(f), h_{2}\right), c\right)$ cannot block $\mu$. 


\subsection{EXISTENCE OF STABLE MATCHING WHEN COUPLES' PREFERENCES}

Suppose $\mu(f)=h$. Because $\left(\mu(f), h_{2}\right) P_{c}^{0}(\mu(f), \mu(m))$, the definition of $P_{m \mid h}^{0}$ implies $h_{2} P_{m \mid h}^{0} \mu(m)$. Since all doctors who propose before $m$ are ranked above $m$ in $P^{0}$ and $m \notin \mu\left(h_{2}\right)$, it must be that $d P^{0} m$ for all $d \in \mu\left(h_{2}\right)$ and $\left|\mu\left(h_{2}\right)\right|=\kappa_{h_{2}}$. By responsiveness of hospitals' preferences, this means $\mu\left(h_{2}\right) \underset{\sim}{P_{h_{2}}}\left(\left(\mu\left(h_{2}\right) \backslash d\right) \cup m\right)$ for all $d \in \mu\left(h_{2}\right)$. However, this contradicts that $\left(\left(\mu(f), h_{2}\right), c\right)$ blocks $\mu$.

This completes the first part of the theorem.

[Part $(i i)]$ Suppose $P_{C}^{0}$ is not responsive for $\mathrm{F}$. We show that there is an extension of $P_{C}^{0}$ with no stable matching. Since $P_{C}^{0}$ does not satisfy responsive for F property, there must exist a couple $c=\{f, m\}$ and two hospitals $h_{1}, h_{2} \in H$ such that $\left(h_{1}, h_{1}\right) P_{c}^{0}\left(h_{2}, h_{1}\right)$ and $h_{2} P_{f}^{0} h_{1}$. Moreover, since $h_{2} P_{f}^{0} h_{1}$, it follows from the definition of RVT that $\left(h_{2}, h_{2}\right) P_{c}^{0}\left(h_{1}, h_{2}\right)$.

Consider a profile $\underset{\sim}{P}$ such that

1. there are doctors $d_{1}, d_{2} \in D \backslash\{f, m\}$ with $f P^{0} d_{1} P^{0} d_{2} P^{0} m$ such that $r_{1}\left(\underset{\sim}{P_{1}}\right)=h_{1}$ and $r_{1}\left(\underset{\sim}{P} d_{2}\right)=h_{2}$,

2. $\mid\left\{d: d P^{0} f\right.$ and $\left.r_{1}(\underset{\sim}{P})=h_{2}\right\}\left|=\kappa_{h_{2}}-2,\right|\left\{d: d P^{0} f\right.$ and $\left.r_{1}\left(\underset{\sim}{P_{d}}\right)=h_{1}\right\} \mid=\kappa_{h_{1}}-2$, and $\mid\left\{d: d P^{0} f\right.$ and $\left.r_{1}(\underset{\sim}{P})=h\right\} \mid=\kappa_{h}$ for all $h \neq h_{1}, h_{2}$, and

3. the preferences of all couples other than $c$ satisfy responsiveness.

Since $\sum_{h \in H} \kappa_{h}=|D|$ by the construction of $\underset{\sim}{P}$, the four bottom-ranked (lowest ranked) doctors in $P^{0}$ are $f, d_{1}, d_{2}, m$. We show that there is no stable matching at $\underset{\sim}{P}$. Assume for contradiction that a matching $\mu$ is stable matching at $\underset{\sim}{P}$. Since $\mu$ is stable at $\underset{\sim}{P}$, by the construction $\underset{\sim}{P}$, it is straight forward that $\mu(d)=r_{1}\left(\underset{\sim}{P_{d}}\right)$ for all $d P^{0} f$.

Because $\mid\left\{d: d P^{0} f\right.$ and $\left.r_{1}(\underset{\sim}{P})=h\right\} \mid=\kappa_{h}$ for all $h \neq h_{1}, h_{2}$, stability of $\mu$ implies that the doctors $f, d_{1}, d_{2}, m$ cannot be matched to any hospital other than $h_{1}$ and $h_{2}$. Moreover, since $\mid\left\{d: d P^{0} f\right.$ and $\left.r_{1}(\underset{\sim}{P})=h_{2}\right\} \mid=\kappa_{h_{2}}-2$ and $\mid\left\{d: d P^{0} f\right.$ and $r_{1}\left(\underset{\sim}{P_{d}}\right)=$ $\left.h_{1}\right\} \mid=\kappa_{h_{1}}-2$, exactly two doctors among $f, d_{1}, d_{2}, m$ must be matched to each of $h_{1}$ and $h_{2}$.

Now, we distinguish the following cases depending on the allocation of the couple $c$ and show that $\mu$ is not stable in any of these cases.

- Suppose $\mu(c)=\left(h_{2}, h_{2}\right)$. 
Then, $\left(h_{2}, d_{2}\right)$ blocks $\mu$ as $r_{1}\left(\underset{\sim}{P_{d_{2}}}\right)=h_{2}$ and $d_{2} P^{0} m$.

- Suppose $\mu(c)=\left(h_{1}, h_{2}\right)$.

Then, $\left(\left(h_{2}, h_{2}\right), c\right)$ blocks $\mu$ as $f P^{0} d_{1} P^{0} d_{2}$, and by RVT, $\left(h_{2}, h_{2}\right) P_{c}^{0}\left(h_{1}, h_{2}\right)$.

- Suppose $\mu(c)=\left(h_{1}, h_{1}\right)$.

Then, $\left(h_{1}, d_{1}\right)$ blocks $\mu$ as $r_{1}\left(\underset{\sim}{P} d_{1}\right)=h_{1}$ and $d_{1} P^{0} m$.

- Suppose $\mu(c)=\left(h_{2}, h_{1}\right)$.

Then, $\left(\left(h_{1}, h_{1}\right), c\right)$ blocks $\mu$ as $f P^{0} d_{1} P^{0} d_{2}$, and by RVT, $\left(h_{1}, h_{1}\right) P_{c}^{0}\left(h_{2}, h_{1}\right)$.

This completes the proof.

\subsection{Existence of stable matching with strong CPI}

In Section 2.4, we have considered the case where couples violate responsiveness for being matched together at some hospital and have provided a necessary and sufficient condition for the existence of stable matchings. In this section, we go beyond RVT and consider arbitrary violation of responsiveness of couples' preferences. In other words, we assume that a couple can have any preference over pairs of hospitals irrespective of the individual preferences of its members. Note that in our model a couple need not be a wife-husband pair, it only represents a pair of doctors who have a joint preference. For instance, a couple might consist of two jealousy/competitive people or two exes, etc. Thus, a couple need not be always willing to stay together, instead it might prefer to stay apart. This justifies our consideration of arbitrary couples' preferences. Note that we still assume that any couple prefers an allocation where each member of the couple is matched to some hospital over an allocation where at least one member of the couple is unmatched. In this setting, we introduce the notion of strong CPI and show that strong CPI is both necessary and sufficient for the existence of a stable matching.

In what follows, we provide a verbal description of the strong CPI property. Suppose that hospitals' preferences satisfy CPI with respect to $P^{0}$. Roughly speaking, strong CPI ensures that the members of each couple are ranked 'very close' to each other. More precisely, it says that (i) if the $m$-member of a couple is not the worst doctor of $D$ according to $P^{0}$ and if there are enough doctors in $D$ to fill (or exceed) the capacity of at least one hospital, then, in fact, the members of that couple must be 
ranked consecutively in $P^{0}$, (ii) otherwise, there can be at most one doctor ranked in-between the members of the couple. Below, we provide the formal definition of this.

Recall that whenever hospitals' preferences are assumed to satisfy CPI with respect to some $P^{0}$, we assume $f P^{0} m$ for any couple $\{f, m\}$. Also, recall that we write $r\left(d, P^{0}\right)=k$ to mean that $d$ has rank $k$ in $P^{0}$, that is, $r_{k}\left(P^{0}\right)=d$.

Definition 2.10. Let hospitals' preferences satisfy CPI with respect to $P^{0}$. Then, $P^{0}$ is said to satisfy strong CPI (SCPI) if for any couple $c=\{f, m\} \in C$,

(i) $r\left(m, P^{0}\right) \neq|D|$ implies either $\left|\left\{d \in D: f P^{0} d P^{0} m\right\}\right|=0$ or $\left|\left\{d \in D: d P^{0} m\right\}\right|<$ $\kappa_{h}$ for all $h \in H$, and

(ii) $r\left(m, P^{0}\right)=|D|$ implies $\left|\left\{d \in D: f P^{0} d P^{0} m\right\}\right| \leq 1$.

A preference $P_{c}$ of a couple $c \in C$ is unrestricted if it is an arbitrary element of $\mathbb{L}\left(\bar{H}^{2}\right)$ satisfying the only requirement that the couple prefers both its members to be matched to some hospital rather than having at least one member unmatched.

Suppose that hospitals' preferences satisfy CPI with respect to some preference $P^{0}$ over the individual doctors. We introduce the notion of extension of this preference $P^{0}$ to preference profiles. As the name suggests, an extension of $P^{0}$ to a preference profile is basically a preference profile where hospitals satisfy CPI with respect to $P^{0}$. By an RVT extension of $P^{0}$, we refer to any preference profile where couples' preferences satisfy RVT, and by an unrestricted extension of $P^{0}$, we refer any preference profile where the couples' preferences are unrestricted. Of course, in both these extensions hospitals' preferences satisfy CPI with respect to $P^{0}$.

Our next theorem provides a sufficient condition for the existence of a stable matching at every unrestricted extension of hospitals' preferences satisfying CPI.

Theorem 2.2. Let hospitals' preferences satisfy CPI with respect to $P^{0}$. If $P^{0}$ satisfies $S C P I$, then a stable matching exists at every unrestricted extension of $P^{0}$.

Proof. Suppose $P^{0}$ satisfies SCPI. We show that there exists a stable matching for every unrestricted extension $\underset{\sim}{P}$ of $P^{0}$. Recall that by our initial assumption on CPI, $m_{i} P^{0} m_{j}$ for all $i, j \in\{1, \ldots, k\}$ such that $i<j$. Since $P^{0}$ satisfies SCPI, this means $f_{i} P^{0} f_{j}$ for all $i, j \in\{1, \ldots, k\}$ such that $i<j$. In the following, we present an algorithm that produces a stable matching at $\underset{\sim}{P}$. 
Algorithm 2: We present the $1^{\text {st }}$ step and a general step of the algorithm.

Step 1: Use SDA to match all the doctors who are ranked above $f_{1}$ according to $P^{0}$. Let $c_{1}$ propose to the hospital(s) $r_{1}\left({\underset{\sim}{c_{1}}}_{1}\right)$, say $\left(h_{1}, h_{2}\right)$. If any member of the couple is rejected, i.e., if $f_{1}$ is rejected by $h_{1}$ and/or $m_{1}$ is rejected by $h_{2}$, then let $c_{1}$ propose to $r_{2}\left(\underset{\sim}{P} c_{1}\right)$. Again, if any member of the couple is rejected, then let $c_{1}$ propose to $r_{3}\left(\underset{\sim}{P} c_{1}\right)$, and so on. Continue this process till both members of the couple are accepted.

Step j: Having matched all the doctors from the top till $c_{j-1}$ in $P^{0}$ in steps 1 to $j-1$, use SDA to match all the doctors that ranked below $c_{j-1}$ and above $c_{j}$ according to $P^{0}$. Let $c_{j}$ propose to $r_{1}\left(\underset{\sim}{P_{c_{j}}}\right)$. If at least one member of the couple is rejected, then let $c_{j}$ propose to $r_{2}\left(\underset{\sim}{P} c_{j}\right)$, and so on. Continue this process till both the members of the couple are accepted.

Continue this process till Step $k-1$. Having matched all the doctors from the top till $c_{k-1}$ according to $P^{0}$ in steps 1 to $k-1$, use SDA to match all the doctors that are ranked below $c_{k-1}$ and above $f_{k}$ according to $P^{0}$. We distinguish the following two cases to match remaining doctors.

Case 1. Suppose there is no single doctor in between $f_{k}$ and $m_{k}$ in $P^{0}$ or $\mid\{d \in D$ : $\left.d P^{0} m_{k}\right\} \mid<\kappa_{h}$ for all $h \in H$. Let $c_{k}$ propose to $r_{1}\left(\underset{\sim}{P} c_{k}\right)$. If at least one member of the couple is rejected, then let $c_{k}$ propose to $r_{2}\left(\underset{\sim}{P_{k}}\right)$, and so on. Continue this process till both the members of the couple are accepted. Finally, match all the remaining doctors using SDA.

Case 2. Suppose there is a single doctor, say $s^{\prime}$, in between $f_{k}$ and $m_{k}$ in $P^{0}$. Note that by SCPI, there cannot be more than one single doctor in between $f_{k}$ and $m_{k}$. Suppose $H^{\prime}$ is the set of hospitals that have at least one remaining vacancy. Let $h^{\prime}$ be the worst hospital in $H^{\prime}$ according to $\underset{\sim}{P_{s^{\prime}}}$ and let $h \in H^{\prime}$ be such that $\left(h, h^{\prime}\right) \underset{\sim}{R_{c_{k}}}\left(h^{\prime \prime}, h^{\prime}\right)$ for all $h^{\prime \prime} \in H^{\prime}$. Then, match $c_{k}$ with $\left(h, h^{\prime}\right)$ and $s^{\prime}$ to the hospital that has a remaining vacancy.

Let $\mu$ be the outcome of Algorithm 2. We show that $\mu$ is stable at $\underset{\sim}{P}$.

Assume for contradiction that $\mu$ is blocked by a hospital and a single doctor or a pair of hospitals and a couple. We complete the proof by considering the two cases of 
Algorithm 2 separately.

Case 1. Suppose Case 1 of Algorithm 2 holds. First, we show that $\mu$ cannot be blocked by $(h, s)$ for some $h \in H$ and $s \in S$. Assume for contradiction that some pair $(h, s)$ blocks $\mu$. By the nature of Algorithm 2, all the doctors that propose before $s$ are ranked above $s$ according to the SCPI $P^{0}$. Moreover, for any $c=\{f, m\} \in C$, if $f P^{0} s$, then by SCPI, $m P^{0} s$. Since $s \notin \mu(h)$, by the nature of Algorithm 2, we have either $\mu(s) \underset{\sim}{P} h$ or $d P^{0} s$ for all $d \in \mu(h)$ and $|\mu(h)|=\kappa_{h}$. Clearly, if $\mu(s){\underset{\sim}{S}}_{s} h$ then $s$ does not block with hospital $h$. On the other hand, if $d P^{0} s$ for all $d \in \mu(h)$ and $|\mu(h)|=\kappa_{h}$, then by responsiveness of hospitals' preferences, we have $\mu(h) P_{\sim}(\mu(h) \backslash d) \cup s$ for all $d \in \mu(h)$. Therefore, hospital $h$ does not block with $s$. This contradicts that $(h, s)$ blocks $\mu$.

Next we show that $\mu$ can not be blocked by $\left(\left(h_{1}, h_{2}\right), c\right)$ for some $h_{1}, h_{2} \in H$ and $c=\{f, m\} \in C$. Note that, we can not have $\mid\left\{d \in D: d P^{0} m\right\}<\kappa_{h}$ for all $h$. This is because, if all the hospitals have enough vacancies to accommodate the couple members, $c$ would not get rejected by any pair of hospitals it applies to and thus, the $c$ would be matched to their top ranked pair of hospitals. Which implies that $c$ cannot block $\mu$.

Therefore, by the nature of Algorithm 2, couple $c$ proposes to $\left(h_{1}, h_{2}\right)$ before proposing to $(\mu(f), \mu(m))$, and some hospital, say $h_{i} \in\left\{h_{1}, h_{2}\right\}$, rejects at least one member of the couple $c$. We distinguish the following two sub-cases.

Case 1.1. Suppose $h_{1} \neq h_{2}$. Since $h_{i}$ rejects a doctor from couple $c$, it must be that $h_{i}$ has no vacancies when $c$ proposes to $\left(h_{1}, h_{2}\right)$. Because $f$ and $m$ are adjacent in $P^{0}$, this means all the doctors in $h_{i}$ are preferred to both $f$ and $m$. Therefore, $h_{i}$ will be worse off by removing a doctor from $\mu\left(h_{i}\right)$ and taking a member from the couple $c$. This contradicts that $\left(\left(h_{1}, h_{2}\right), c\right)$ blocks $\mu$.

Case 1.2. Suppose $h_{1}=h_{2}$. Because $h_{1}$ rejects at least one member of $c$, it must be that $h_{1}$ has less than two vacancies when $c$ proposes to $\left(h_{1}, h_{1}\right)$. Let $D^{\prime}$ be the set of doctors that are present in $h_{1}$ at the time when $c$ makes the proposal to $\left(h_{1}, h_{1}\right)$. By SCPI and the nature Algorithm 2, this means each doctor in $D^{\prime}$ is preferred to both the doctors of the couple $c$. Again, by the nature of Algorithm 2, it follows that $D^{\prime} \subseteq \mu\left(h_{1}\right)$. This means $h_{1}$ must release some doctors from $D^{\prime}$ in order to block with $c$. Therefore, $h_{1}$ will be worse off by removing a doctor from $D^{\prime}$ in order to take a member of the couple. This contradicts that $\left(\left(h_{1}, h_{1}\right), c\right)$ blocks $\mu$. 
This completes the proof of Case 1 of Theorem 2.2.

Case 2. Suppose Case 2 of Algorithm 2 holds. Note that after matching all the doctors from the top till $f_{k}$ in $P^{0}$, we have exactly three vacancies left. To see this, recall that each couple and each single doctor prefer to be matched to any hospital than being unmatched. Therefore, all the doctors ranked above $f_{k}$ will be matched to some hospital. Since $\sum_{h \in H} \kappa_{h}=|D|$, it follows that total number of remaining vacancies in hospitals in $H^{\prime}$ is 3 .

Note that, by similar argument as for Case 1, (i) $\mu$ cannot be blocked by $(h, s)$ for any $s P^{0} f_{k}$, and (ii) $\mu$ cannot be blocked by $\left(\left(h_{1}, h_{2}\right), c\right)$ for any $c$ such that $c \neq c_{k}$.

First, we show $\mu$ cannot be blocked by $\left(h, s^{\prime}\right)$, where $s^{\prime}$ is the unique single doctor inbetween $f_{k}$ and $m_{k}$. Suppose not. Since $d P^{0} s^{\prime}$ for all $d \neq m_{k}$, it follows that $h \in H^{\prime}$. By Algorithm 2, $\mu\left(s^{\prime}\right) \in H^{\prime}$ and $\mu\left(m_{k}\right)$ is the worst hospital in $H^{\prime}$ according to ${\underset{\sim}{s^{\prime}}}_{\text {. Since }}$ $\mu$ is blocked by $\left(h, s^{\prime}\right)$, we have $h \underset{\sim}{P_{s^{\prime}}} \mu\left(s^{\prime}\right) \underset{\sim}{R_{s^{\prime}}} \mu\left(m_{k}\right)$. This means $h \neq \mu\left(m_{k}\right)$. We now show that $h \neq \mu\left(f_{k}\right)$. Assume for contradiction, $h=\mu\left(f_{k}\right)$. By our earlier argument, since $h \neq \mu\left(m_{k}\right), \mu\left(f_{k}\right)=\mu\left(m_{k}\right)$ implies $h \neq \mu\left(f_{k}\right)$. Suppose $\mu\left(f_{k}\right) \neq \mu\left(m_{k}\right)$. This means all the doctors in $\mu\left(f_{k}\right)$ are ranked above $s^{\prime}$ according to $P^{0}$, contradicting the fact that $\mu\left(f_{k}\right)$ and $s^{\prime}$ block $\mu$. This shows $h \neq \mu\left(f_{k}\right)$. By the definition of Algorithm $2, h \in\left\{\mu\left(s^{\prime}\right), \mu\left(f_{k}\right), \mu\left(m_{k}\right)\right\}$. Since $h \notin\left\{\mu\left(f_{k}\right), \mu\left(m_{k}\right)\right\}$, it must be that $h=\mu\left(s^{\prime}\right)$, and hence $h$ and $s^{\prime}$ can not block.

Now, we show that $\mu$ cannot be blocked by $\left(\left(h_{1}, h_{2}\right), c_{k}\right)$ for some $h_{1}, h_{2} \in H$. Since $d P^{0} f_{k}$ for all $d \notin\left\{s^{\prime}, m_{k}\right\}$, it follows from Algorithm 2 and the definition of $H^{\prime}$ that $h_{1}, h_{2} \in H^{\prime}$. We complete the proof by distinguishing the following two cases.

Case 2.1. Suppose $h_{2}=\mu\left(m_{k}\right)$. By Algorithm 2, $\mu\left(c_{k}\right) \underset{\sim}{R_{c_{k}}}\left(h_{1}, h_{2}\right)$ for all $h_{1} \in H^{\prime}$. Therefore, $c_{k}$ will not block with $\left(h_{1}, h_{2}\right)$.

Case 2.2. Suppose $h_{2} \neq \mu\left(m_{k}\right)$. By Algorithm 2, this means all the doctors in $h_{2}$ are preferred to $m_{k}$ according to $P^{0}$. Therefore, $h_{2}$ will not block with $m_{k}$.

This completes the proof of Case 2 of Theorem 2.2.

Since Case 1 and Case 2 are exhaustive, this completes the proof of Theorem 2.2. 
Now, we look at the converse of Theorem 2.2. It states the following: If hospitals' preferences satisfy CPI but violates SCPI, then there always exists an unrestricted extension of $P^{0}$ at which there is no stable matching.

However, we prove a stronger version of this converse, where we show that when hospitals' preferences satisfy CPI but violates SCPI, one can find even an RVT extension of $P^{0}$ where there is no stable matching. Thus, under the said assumption, one does not have to look for an unrestricted extension to get hold of a profile with no stable matching.

Theorem 2.3. Let hospitals' preferences satisfy CPI with respect to $P^{0}$. If $P^{0}$ does not satisfy SCPI, then there always exists an RVT extension of $P^{0}$ at which there is no stable matching.

Proof. Suppose a CPI $P^{0}$ does not satisfy SCPI. We show that there exists an RVT extension of $P^{0}$ with no stable matching. Since $P^{0}$ does not satisfy SCPI, one of the following two cases must happen:

Case 1. There is a couple $c=\{f, m\}$ such that $r\left(m, P^{0}\right) \neq|D|,\left|\left\{d \in D: f P^{0} d P^{0} m\right\}\right|>$ 0 and $\left|\left\{d \in D: d P^{0} m\right\}\right| \geq \kappa_{h}$ for some $h \in H$. Thus, there exist doctors $d_{1}, d_{2}$ such that $f P^{0} d_{1} P^{0} m P^{0} d_{2}$ and a hospital $h_{1}$ such that $\left|\left\{d \in D: d P^{0} m\right\}\right| \geq \kappa_{h_{1}}$.

Case 2. There is a couple $c=\{f, m\}$ such that $r\left(m, P^{0}\right)=|D|$ and $\left|\left\{d \in D: f P^{0} d P^{0} m\right\}\right|>1$. In other words, there exist doctors $d_{1}, d_{2}$ such that $f P^{0} d_{1} P^{0} d_{2} P^{0} m$.

In the following, we present an RVT extension of $P^{0}$ with no stable matching for both Case 1 and Case 2.

Take hospitals $h_{1}, h_{2} \in H$ and consider a preference profile $\underset{\sim}{P}$ such that

1. $r_{1}(\underset{\sim}{P})=r_{1}\left({\underset{\sim}{d_{2}}}_{d_{2}}\right)=h_{2}$ and $r_{1}(\underset{\sim}{P})=r_{1}\left(\underset{\sim}{P_{d_{1}}}\right)=h_{1}$,

2. $r_{2}(\underset{\sim}{P})=r_{2}\left(\underset{\sim}{P} d_{2}\right)=h_{1}$ and $r_{2}(\underset{\sim}{P})=r_{2}\left(\underset{\sim}{P} d_{1}\right)=h_{2}$,

3. $\left(h_{1}, h_{1}\right) \underset{\sim}{P_{c}}\left(h_{2}, h_{1}\right)$ and $\left(h_{1}, h_{1}\right) \underset{\sim}{P}\left(h_{2}, h_{2}\right)$,

4. $\left(h_{1}, h_{2}\right) \underset{\sim}{P_{c}}\left(h, h^{\prime}\right)$ for all $h, h^{\prime} \in H$ such that $\left(h, h^{\prime}\right)$ does not belong to the set $\left\{\left(h_{1}, h_{1}\right),\left(h_{2}, h_{2}\right),\left(h_{2}, h_{1}\right),\left(h_{1}, h_{2}\right)\right\}$,

5. preference $\underset{\sim}{P_{c}}$ satisfies responsiveness for all pairs of hospitals other than $\left(h_{1}, h_{1}\right)$, 
6. preferences of all couples other than $c$ satisfy responsiveness,

7. $\mid\left\{d: r_{1}(\underset{\sim}{P})=h_{1}\right.$ and $\left.d P^{0} m\right\} \mid=\kappa_{h_{1}}-1$, and

8. for all $d \notin\left\{f, m, d_{1}, d_{2}\right\},\left|\left\{d: r_{1}(\underset{\sim}{P})=h_{2}\right\}\right|=\kappa_{h_{2}}-2$ and $\left|\left\{d: r_{1}\left({\underset{\sim}{d}}_{d}\right)=h\right\}\right|=$ $\kappa_{h}$ for all $h \neq h_{1}, h_{2}$.

Note that the assumption made in condition 7 is possible as $\left|\left\{d \in D: d P^{0} m\right\}\right|=\kappa_{h_{1}}$ and $f P^{0} m$. However, $r_{1}\left(\underset{\sim}{P_{f}}\right) \neq h_{1}$. We show that there is no stable matching at $\underset{\sim}{P}$ for both Case 1 and Case 2. Assume for contradiction that $\mu$ is a stable matching at $\underset{\sim}{P}$. Note that by the construction of $\underset{\sim}{P}$, for all doctors $d$ such that $d P^{0} f$, we must have $\mu(d)=r_{1}(\underset{\sim}{P})$. In the following claim, we show that $\mu(d) \in\left\{h_{1}, h_{2}\right\}$ for all $d \in\left\{f, m, d_{1}, d_{2}\right\}$.

Claim 2.1. For all $d \in\left\{f, m, d_{1}, d_{2}\right\}, \mu(d) \in\left\{h_{1}, h_{2}\right\}$.

Proof. First, we show $\mu(d) \in\left\{h_{1}, h_{2}\right\}$ for $d \in\{f, m\}$. Suppose $\mu(d)=h^{\prime}$ for some $d \in\{f, m\}$ and some $h^{\prime} \notin\left\{h_{1}, h_{2}\right\}$. We complete the proof for the case where $\mu(m)=$ $h^{\prime}$, the same for the case $\mu(f)=h^{\prime}$ follows from similar arguments. Let $\mu(c)=\left(h, h^{\prime}\right)$ for some $h \in H$. Consider the matchings $\left(h, h_{1}\right)$ and $\left(h, h_{2}\right)$ of the couple $c$. Note that by responsiveness, $\left(h, h_{1}\right) \underset{\sim}{P_{c}}\left(h, h^{\prime}\right)$ and $\left(h, h_{2}\right){\underset{\sim}{c}}_{c}\left(h, h^{\prime}\right)$. Further, since $\sum_{h \in H} \kappa_{h}=|D|$ and $\mu(d)=r_{1}(\underset{\sim}{P})$ for all doctors $d$ such that $d P^{0} f, \mu(m)=h^{\prime}$ implies that there must be a doctor $d^{\prime}$ with $m P^{0} d^{\prime}$ such that either $d^{\prime} \in \mu\left(h_{1}\right)$ or $d^{\prime} \in \mu\left(h_{2}\right)$. This means couple $c$ blocks $\mu$ with either $\left(h, h_{1}\right)$ or $\left(h, h_{2}\right)$ contradicting the stability of $\mu$. Therefore, $\mu(d) \in\left\{h_{1}, h_{2}\right\}$ for all $d \in\{f, m\}$.

Now, we show $\mu(d) \in\left\{h_{1}, h_{2}\right\}$ for $d \in\left\{d_{1}, d_{2}\right\}$. Suppose $\mu(d)=h^{\prime}$ for some $d \in$ $\left\{d_{1}, d_{2}\right\}$ and some $h^{\prime} \notin\left\{h_{1}, h_{2}\right\}$. Since $\mu(d) \in\left\{h_{1}, h_{2}\right\}$ for all $d \in\{f, m\}$ and $\mu(d)=$ $r_{1}\left({\underset{\sim}{d}}_{d}\right)$ for all doctors $d$ such that $d P^{0} f$, there must be a doctor $d^{\prime}$ with $d_{2} P^{0} d^{\prime}$ such that either $d^{\prime} \in \mu\left(h_{1}\right)$ or $d^{\prime} \in \mu\left(h_{2}\right)$. Because $r_{k}\left(\underset{\sim}{P_{d}}\right) \in\left\{h_{1}, h_{2}\right\}$ for all $k=1,2$, if $\mu\left(d^{\prime}\right)=h_{1}$, then $d$ blocks $\mu$ with $h_{1}$, and if $\mu\left(d^{\prime}\right)=h_{2}$, then $d$ blocks $\mu$ with $h_{2}$. This contradicts the stability of $\mu$. Therefore, $\mu(d) \in\left\{h_{1}, h_{2}\right\}$ for all $d \in\left\{d_{1}, d_{2}\right\}$.

This completes the proof of Claim 1 .

Now, we distinguish the following cases depending on the allocation of couple $c$ and show that $\mu$ is not stable for each of these cases.

- Suppose $\mu(c)=\left(h_{1}, h_{1}\right)$. 
Since $\mid\left\{d: r_{1}(\underset{\sim}{P})=h_{1}\right.$ and $\left.d P^{0} f\right\} \mid=\kappa_{h_{1}}-2$ and $f P^{0} d_{1}$, thus $d_{1} \notin \mu\left(h_{1}\right)$. Because $h_{1} \underset{\sim}{P} d_{1} h_{2}$ and $d_{1} P^{0} m$, this means $\left(h_{1}, d_{1}\right)$ blocks $\mu$.

- Suppose $\mu(c)=\left(h_{2}, h_{1}\right)$.

Then, $\left(\left(h_{1}, h_{1}\right), c\right)$ blocks $\mu$ as $f P^{0} d_{1} P^{0} d_{2}$ and $\left(h_{1}, h_{1}\right) \underset{\sim}{P_{c}}\left(h_{2}, h_{1}\right)$.

- Suppose $\mu(c)=\left(h_{2}, h_{2}\right)$.

By the construction of $\underset{\sim}{P},\left(h_{1}, h_{1}\right) \underset{\sim}{P}\left(h_{2}, h_{2}\right)$ and $h_{2} \underset{\sim}{P_{2}} h_{1}$. If Case 1 holds, then $\left(\left(h_{1}, h_{1}\right), c\right)$ blocks $\mu$ as $f P^{0} d_{1}$ and $m P^{0} d_{2}$. On the other hand, if Case 2 holds, then $\left(h_{2}, d_{2}\right)$ blocks $\mu$ as $d_{2} P^{0} m$.

- Suppose $\mu(c)=\left(h_{1}, h_{2}\right)$.

Since $h_{2} P_{f} h_{1}$, by RVT, $\left(h_{2}, h_{2}\right) \underset{\sim}{P_{c}}\left(h_{1}, h_{2}\right)$. This, together with the fact that $f P^{0} d_{1} P^{0} d_{2}$, means $\mu$ is blocked by $\left(\left(h_{2}, h_{2}\right), c\right)$.

This completes the proof of Theorem 2.3.

The following corollary is immediate from Theorem 2.2 and (the stronger version of) Theorem 2.3.

Corollary 2.1. Let hospitals' preferences satisfy CPI with respect to $P^{0}$. Then a stable matching is guaranteed at every unrestricted extension of $P^{0}$ if and only if $P^{0}$ satisfies SCPI.

\subsection{Matching market with non-identical hospital pref- erences}

In both sections 2.4 and 2.5, we have assumed that the hospitals have identical preferences over the doctors. In this section, we explore the situations where this condition is relaxed. From Example 2.1, we know that a stable matching does not exist under CPI, when couples' preferences satisfy RVT. However, to strengthen Theorem 2.1, in the case when hospitals do not have CPI, we first show by means of the following example that if hospitals' preferences do not satisfy CPI and couples' preferences satisfy RVT, then stable matching might not exist at every preference profile. 
Example 2.2. Consider the matching problem where $H=\left\{h_{1}, h_{2}, h_{3}\right\}$ with $\kappa_{h_{1}}=$ $\kappa_{h_{2}}=\kappa_{h_{3}}=2, D=\left\{f, m, s_{1}, s_{2}, s_{3}, s_{4}\right\}$, and there is exactly one couple $c=\{f, m\}$ in C.

The preferences of the hospitals over individual doctors and those of the individual doctors and the couple are given in Table 2.2. The couple's preference over pairs of hospitals where one member is matched and the other one is unmatched is not shown in the table, but assumed to be responsive and ranked below the shown pairs.

\begin{tabular}{cccccccccc}
\hline$P_{h_{1}}$ & $P_{h_{2}}$ & $P_{h_{3}}$ & $P_{s_{1}}$ & $P_{s_{2}}$ & $P_{s_{3}}$ & $P_{s_{4}}$ & $P_{f}$ & $P_{m}$ & $P_{c}$ \\
\hline$s_{3}$ & $s_{4}$ & $s_{3}$ & $h_{2}$ & $h_{3}$ & $h_{1}$ & $h_{2}$ & $h_{1}$ & $h_{2}$ & $\left(h_{1}, h_{2}\right)$ \\
$s_{4}$ & $s_{3}$ & $s_{4}$ & $h_{1}$ & $h_{1}$ & $h_{2}$ & $h_{1}$ & $h_{3}$ & $h_{1}$ & $\left(h_{1}, h_{1}\right)$ \\
$s_{1}$ & $f$ & $m$ & $h_{3}$ & $h_{2}$ & $h_{3}$ & $h_{3}$ & $h_{2}$ & $h_{3}$ & $\left(h_{1}, h_{3}\right)$ \\
$f$ & $m$ & $f$ & & & & & & & $\left(h_{3}, h_{3}\right)$ \\
$m$ & $s_{1}$ & $s_{1}$ & & & & & & & $\left(h_{3}, h_{2}\right)$ \\
$s_{2}$ & $s_{2}$ & $s_{2}$ & & & & & & & $\left(h_{3}, h_{1}\right)$ \\
& & & & & & & & & $\left(h_{2}, h_{2}\right)$ \\
& & & & & & & & & $\left(h_{2}, h_{1}\right)$ \\
& & & & & & & & & $\left(h_{2}, h_{3}\right)$ \\
\hline
\end{tabular}

Table 2.2: Non-Identical Hospital Preferences

We show that there is no stable matching at this preference profile. Assume for contradiction that $\mu$ is a stable matching at that profile. Since $\mu$ is stable, $r_{1}\left(P_{S_{3}}\right)=h_{1}$ and $r_{1}\left(P_{h_{1}}\right)=s_{3}$ imply $\mu\left(s_{3}\right)=h_{1}$. Using similar logic, stability of $\mu$ implies $\mu\left(s_{4}\right)=h_{2}$. Moreover, because $s_{1} P_{h} s_{2}$ for all $h \in H$, we must have $\mu\left(s_{1}\right) R_{s_{1}} \mu\left(s_{2}\right)$. Now, we consider all possible cases of couples' matching satisfying the above criteria and show that $\mu$ is blocked in each of those cases.

- Suppose $\mu(c)=\left(h_{1}, h_{2}\right)$. Since $h_{1} P_{s_{1}} h_{3}$ and $s_{1} P_{h_{1}} f, \mu$ is blocked by $\left(h_{1}, s_{1}\right)$.

- Suppose $\mu(c)=\left(h_{1}, h_{3}\right)$. Since $\left(h_{1}, h_{2}\right) P_{c}\left(h_{1}, h_{3}\right)$ and $m P_{h_{2}} s_{1}, \mu$ is blocked by $\left(\left(h_{1}, h_{2}\right), c\right)$.

- Suppose $\mu(c)=\left(h_{3}, h_{3}\right)$. Since $\left(h_{1}, h_{2}\right) P_{c}\left(h_{3}, h_{3}\right), m P_{h_{2}} s_{1}$, and $f P_{h_{1}} s_{2}, \mu$ is blocked by $\left(\left(h_{1}, h_{2}\right), c\right)$.

- Suppose $\mu(c)=\left(h_{3}, h\right)$ for some $h \in\left\{h_{1}, h_{2}\right\}$. Since $\left(h_{3}, h_{3}\right) P_{c}\left(h_{3}, h\right)$ and $m P_{h_{3}} s_{2}, \mu$ is blocked by $\left(\left(h_{3}, h_{3}\right), c\right)$.

- Suppose $\mu(c)=\left(h_{2}, h_{1}\right)$. Since $h_{1} P_{s_{1}} h_{3}$ and $s_{1} P_{h_{1}} m, \mu$ is blocked by $\left(h_{1}, s_{1}\right)$. 
- Suppose $\mu(c)=\left(h_{2}, h_{3}\right)$. Since $\left(h_{3}, h_{3}\right) P_{c}\left(h_{2}, h_{3}\right)$ and $f P_{h_{3}} s_{2}, \mu$ is blocked by $\left(\left(h_{3}, h_{3}\right), c\right)$.

Therefore, there is no stable matching at the preference profile given in Table 2.2.

In view of Example 2.2, we look for restrictions on a preference profile in order to assure the existence of stable matching when hospitals can have different preferences over doctors. We find two natural candidates for this purpose as (i) the responsive for $F$ property of couples' preferences and (ii) common preference over couple members.

Recall that a collection of preferences $P_{C}^{0}$ satisfies responsive for $F$ property if for all $c=\{f, m\} \in C$ and all $h, h^{\prime} \in H,(h, h) P_{c}^{0}\left(h^{\prime}, h\right)$ implies $h P_{f}^{0} h^{\prime}$.

Common preference over couple members is a condition on hospitals' preferences. It says that, for every couple, each hospital prefers the member from $F$ to the member from $M$. Below, we provide a formal definition.

By $P_{H}^{0}$, we denote a collection of preferences $\left\{P_{h}^{0}\right\}_{h \in H}$ of hospitals in $H$.

Definition 2.11. A collection of preferences $P_{H}^{0}=\left\{P_{h}^{0}\right\}_{h \in H}$ of hospitals is said to satisfy Common Preference over Couple members $(C P C)$ if $f P_{h}^{0} m$ for all $c=\{f, m\} \in$ $C$ and all $h \in H$.

Although we find responsive for F property of couples' preferences and CPC property of hospitals' preferences natural candidates for ensuring a stable matching at every profile, Example 2.2 shows they are not sufficient. In view of this, we proceed to strengthen the responsive for F property of couples' preferences. We do this by introducing the notion of almost everywhere responsiveness. This is a restriction on the preferences of non-single doctors and couples. A verbal description of this restriction is given below which is followed by a formal definition.

Take a couple $c=\{f, m\}$ and consider an RVT preference $P_{c}^{0}$ of the couple $c$. Then, $P_{c}^{0}$ satisfies almost everywhere responsiveness property if it satisfies responsiveness over all pairs of hospitals except $\left(r_{1}\left(P_{f}^{0}\right), r_{1}\left(P_{f}^{0}\right)\right)$ and it violates responsiveness for togetherness only when both members of the couple get a position at $r_{1}\left(P_{f}^{0}\right)$. Note that this means couples' preferences satisfy responsiveness with respect to $P_{f}^{0}$, and hence it implies responsive for $F$ property.

Recall that by $P_{C}^{0}$, we denote a collection of preferences of non-single doctors and couples such that $P_{c}^{0}$ satisfies RVT for each couple $c$. 
Definition 2.12. A collection of preferences $P_{C}^{0}$ satisfies almost everywhere responsiveness property $(A E R)$ if for all $c=\{f, m\} \in C$, there exists a responsive preference $P_{c}$ with respect to $P_{f}^{0}$ and $P_{m}^{0}$ such that for all pairs of hospitals $\left(h_{1}, h_{2}\right),\left(h_{3}, h_{4}\right) \in$ $(\bar{H} \times \bar{H}) \backslash\left(r_{1}\left(P_{f}^{0}\right), r_{1}\left(P_{f}^{0}\right)\right)$, we have $\left(h_{1}, h_{2}\right) P_{c}\left(h_{3}, h_{4}\right)$ if and only if $\left(h_{1}, h_{2}\right) P_{c}^{0}\left(h_{3}, h_{4}\right)$.

For a given collection of preferences $\left(P_{C}^{0}, P_{H}^{0}\right)$, an extension of $\left(P_{C}^{0}, P_{H}^{0}\right)$ refers to any preference profile where the preferences of couples and doctors in couples are as in $P_{C}^{0}$ and the preferences of hospitals are as in $P_{H}^{0}$.

The following theorem shows that the existence of a stable matching is guaranteed at a preference profile if hospitals' preferences satisfy CPC and couples preferences satisfy AER.

Theorem 2.4. For a given collection of preferences $\left(P_{C}^{0}, P_{H}^{0}\right)$, a stable matching is guaranteed at every extension of $\left(P^{0}, P_{H}^{0}\right)$ if $P_{H}^{0}$ satisfies $C P C$ and $P_{C}^{0}$ satisfies AER.

Proof. The proof of Theorem 2.4 is constructive. Suppose $\left(P_{M, F, C}^{0}, P_{H}^{0}\right)$ satisfies CPC and AER property. We show that every extension $\underset{\sim}{P}$ of $\left(P_{M, F, C}^{0}, P_{H}^{0}\right)$ has a stable matching.

Take an extension $\underset{\sim}{P}$ of $\left(P_{M, F, C}^{0}, P_{H}^{0}\right)$. For a given couple $c=\{f, m\}$ and a given hospital $h \in H$, define the conditional preference of $m$ given $h$, denoted by $P_{m \mid h}^{0}$, as an element of $\mathbb{L}(\bar{H})$ such that $h^{\prime} P_{m \mid h}^{0} h^{\prime \prime}$ if and only if $\left(h, h^{\prime}\right) P_{c}^{0}\left(h, h^{\prime \prime}\right)$. Let $r_{1}\left(P_{f}^{0}\right)$ is equal to $h_{f}$.

In the following, we present an algorithm that produces a stable matching at $\underset{\sim}{P}$.

Algorithm 3: Use DPDA where every single doctor $s \in S$ proposes according to ${\underset{\sim}{s}}_{s}$ and for every couple $c=\{f, m\}, f$ proposes according to $P_{f}^{0}$ and $m$ proposes according to $P_{m \mid h_{f}}^{0}$.

Let $\mu$ be the outcome of Algorithm 3. The following lemma is straight forward, however for the sake of completeness, we provide its proof.

Lemma 2.1. Suppose a doctor $d$ is rejected by a hospital h at some stage of Algorithm 3. Then, $(h, d)$ cannot block $\mu$. 
Proof. Since $h$ rejected $d$ during some stage of Algorithm 3, it must be that hospital $h$ had $\kappa_{h}$ many proposals from doctors that are better than $d$ according to $P_{h}^{0}$ at the time when $h$ rejected $d$. Therefore, by the nature of DPDA, all the doctors that are matched to $h$ at the end of Algorithm 3 must be better than $d$ according to $P_{h}^{0}$. So, $h$ will not block with $d$. This completes the proof of the lemma.

Now, we show that $\mu$ is stable at $\underset{\sim}{P}$.

First, we show that $\mu$ cannot be blocked by $(h, s)$ for some $h \in H$ and $s \in S$. Assume for contradiction that some pair $(h, s)$ blocks $\mu$. Since $s \notin \mu(h)$, this means either $\mu(s) \underset{\sim}{P_{s}} h$ or $s$ was rejected by $h$ at some stage of the algorithm. Clearly, if $\mu(s){\underset{\sim}{s}}_{s} h$ then $s$ does not block with $h$. On the other hand, if $s$ proposed to $h$ and was rejected by $h$ at an earlier stage, then by Lemma $2.1,(h, s)$ cannot block $\mu$.

Now, we show that $\mu$ cannot be blocked by $\left(\left(h_{1}, h_{2}\right), c\right)$ for some $h_{1}, h_{2} \in H$ and $c \in C$. Assume for contradiction that $\left(\left(h_{1}, h_{2}\right), c\right)$ blocks $\mu$ for some $h_{1}, h_{2} \in H$ and $c \in C$. We distinguish the following two cases.

Case 1. Suppose $\mu(f)=h_{f}$.

First, we show that $\mu(m) \neq h_{2}$. To the contrary, suppose $\mu(m)=h_{2}$. By the AER property, $\left(h_{1}, h_{2}\right) P_{c}^{0}\left(h_{f}, h_{2}\right)$ implies $h_{1} P_{f}^{0} h_{f}$, which contradicts the fact that $h_{f}=r_{1}\left(P_{f}^{0}\right)$.

Next, we show that $\left(h_{f}, h_{2}\right) P_{c}^{0}\left(h_{f}, \mu(m)\right)$. Since $\left(\left(h_{1}, h_{2}\right), c\right)$ blocks $\mu$ and $\mu(f)=h_{f}$, $\left(h_{1}, h_{2}\right) P_{c}^{0}\left(h_{f}, \mu(m)\right)$. Therefore, if $h_{1}=h_{f}$, then there is nothing to prove. Suppose $h_{1} \neq h_{f}$. Then, by the AER property, we have $\left(h_{f}, h_{2}\right) P_{c}^{0}\left(h_{1}, h_{2}\right)$. Since $\left(h_{1}, h_{2}\right) P_{c}^{0}\left(h_{f}, \mu(m)\right)$ and $\mu(m) \neq h_{2}$, this implies $\left(h_{f}, h_{2}\right) P_{c}^{0}\left(h_{f}, \mu(m)\right)$.

Now, we show that $\left(\left(h_{f}, h_{2}\right), c\right)$ cannot block $\mu$. Since $\left(\left(h_{1}, h_{2}\right), c\right)$ blocks $\mu$ and $\mu(f)=h_{f}$, it follows that $\left(\left(h_{f}, h_{2}\right), c\right)$ also blocks $\mu$. By the definition of $P_{m \mid h_{f}}^{0}$, $\left(h_{f}, h_{2}\right) P_{c}^{0}\left(h_{f}, \mu(m)\right)$ implies $h_{2} P_{m \mid h_{f}}^{0} \mu(m)$. Therefore, by the definition of Algorithm 3 , it must be that $m$ proposed to $h_{2}$ and got rejected at an earlier stage of Algorithm 3. Hence, by Lemma 2.1, $\left(\left(h_{f}, h_{2}\right), c\right)$ cannot block $\mu$. This completes the proof for Case 1.

Case 2. Suppose $\mu(f) \neq h_{f}$. 
Since Algorithm 3 uses DPDA where $f$ proposes according to $P_{f}^{0}$, by the property of DPDA, $f$ cannot block according to the preference $P_{f}^{0}$. This, together with the fact that $\left(\left(h_{1}, h_{2}\right), c\right)$ blocks $\mu$, which in particular means that $h_{1}$ blocks with $f$, implies $\mu(f) R_{f}^{0} h_{1}$. Since $r_{1}\left(P_{f}^{0}\right)=h_{f}$ and $\mu(f) \neq h_{f}$, we have $h_{f} P_{f}^{0} \mu(f)$. Because $\mu(f) R_{f}^{0} h_{1}$, this means $h_{1} \neq h_{f}$. By the AER property, $P_{c}^{0}$ is responsive over all pairs of hospitals except $\left(h_{f}, h_{f}\right)$. As $\mu(f) R_{f}^{0} h_{1},\left(h_{1}, h_{2}\right) P_{c}^{0}(\mu(f), \mu(m))$ and the AER property imply $h_{2}=h_{f}$ or $h_{2} P_{m}^{0} \mu(m)$. Because $\mu(f) \neq h_{f}$, it must be that $\left|\mu\left(h_{f}\right)\right|=\kappa_{h_{f}}$ and $d P_{h_{f}}^{0} f$ for all $d \in \mu\left(h_{f}\right)$. Since $f P_{h}^{0} m$ for all $h \in H$ by the CPC property, this means $d P_{h_{f}}^{0} m$ for all $d \in \mu\left(h_{f}\right)$. Therefore, $\mu(m) \neq h_{f}$. Moreover, since $\left(\left(h_{1}, h_{2}\right), c\right)$ blocks $\mu$ and $d P_{h_{f}}^{0} m$ for all $d \in \mu\left(h_{f}\right)$, it follows that $h_{2} \neq h_{f}$. Thus, we have $h_{2} P_{m}^{0} \mu(m)$ which implies $h_{2} \neq \mu(m)$. Since $\mu(m), h_{2}, h_{f}$ are all distinct, $h_{2} P_{m}^{0} \mu(m)$ implies $h_{2} P_{m \mid h_{f}}^{0} \mu(m)$. So, it must be that $m$ proposed to $h_{2}$ and got rejected at an earlier stage of the algorithm. By Lemma 2.1, this means $\left(\left(h_{1}, h_{2}\right), c\right)$ cannot block $\mu$. This completes the proof for Case 2 .

Since Case 1 and Case 2 are exhaustive, this completes the proof of Theorem 2.4.

In the following examples, we show that the conditions CPC and AER are almost necessary for Theorem 2.4. In particular, we show that there always exists a preference extension with no stable matching if a preference profile satisfies one of the two conditions and violates the other 'slightly' (in an appropriate sense).

Example 2.3. In this example, we show that if a preference profile satisfies CPC but violates AER slightly, then there exists a preference extension with no stable matching.

Consider the matching problem where the set of hospitals, their capacities, and the set of doctors are as given in Example 2.3. The preferences of the hospitals over individual doctors and those of the individual doctors and the couple are given in Table 2.3. The couple's preference over pairs of hospitals where one member is matched and the other one is unmatched is not shown in the table, but assumed to be responsive and ranked below the shown pairs. Note that the hospitals' preferences satisfy CPC but couple's preference does not satisfy AER. Further, note that this is a slight violation of AER, because responsiveness is violated in order for the couple to be together only at the second ranked hospital according to $P_{f}$.

The proof of the fact that there is no stable matching at the preference profile in Table 2.3 is similar to that for the case of Example 2.2, and hence is omitted. 


\begin{tabular}{cccccccccc}
\hline$P_{h_{1}}$ & $P_{h_{2}}$ & $P_{h_{3}}$ & $P_{s_{1}}$ & $P_{s_{2}}$ & $P_{s_{3}}$ & $P_{s_{4}}$ & $P_{f}$ & $P_{m}$ & $P_{c}$ \\
\hline$s_{3}$ & $s_{4}$ & $s_{3}$ & $h_{2}$ & $h_{3}$ & $h_{1}$ & $h_{2}$ & $h_{1}$ & $h_{2}$ & $\left(h_{1}, h_{2}\right)$ \\
$s_{4}$ & $s_{3}$ & $s_{4}$ & $h_{1}$ & $h_{1}$ & $h_{2}$ & $h_{1}$ & $h_{3}$ & $h_{1}$ & $\left(h_{1}, h_{1}\right)$ \\
$s_{1}$ & $f$ & $f$ & $h_{3}$ & $h_{2}$ & $h_{3}$ & $h_{3}$ & $h_{2}$ & $h_{3}$ & $\left(h_{1}, h_{3}\right)$ \\
$f$ & $m$ & $m$ & & & & & & & $\left(h_{3}, h_{3}\right)$ \\
$m$ & $s_{1}$ & $s_{1}$ & & & & & & & $\left(h_{3}, h_{2}\right)$ \\
$s_{2}$ & $s_{2}$ & $s_{2}$ & & & & & & & $\left(h_{3}, h_{1}\right)$ \\
& & & & & & & & & $\left(h_{2}, h_{2}\right)$ \\
& & & & & & & & & $\left(h_{2}, h_{1}\right)$ \\
& & & & & & & & & $\left(h_{2}, h_{3}\right)$ \\
\hline
\end{tabular}

Table 2.3: Violation of AER under CPC

Example 2.4. In this example, we show that if a preference profile satisfies AER but violates $\mathrm{CPC}$ slightly, then there exists a preference extension with no stable matching.

Consider the matching problem where $H=\left\{h_{1}, h_{2}\right\}$ with $\kappa_{h_{1}}=\kappa_{h_{2}}=2, D=$ $\left\{f, m, s_{1}, s_{2}\right\}$, and there is exactly one couple $c=\{f, m\}$ in $C$. The preferences of the hospitals over individual doctors, and those of the individual doctors and the couple are given in Table 2.4. The couple's preference over pairs of hospitals where one member is matched and the other one is unmatched is not shown in the table, but assumed to be responsive and ranked below the shown pairs. Note that, couple's preference satisfies AER but hospitals' preferences do not satisfy CPC. Further, note that this is a slight variation from $\mathrm{CPC}$, because the relative position of the members of the couple is switched and the ranking of single doctors remains the same in both the hospitals.

\begin{tabular}{ccccccc}
\hline$P_{h_{1}}$ & $P_{h_{2}}$ & $P_{s_{1}}$ & $P_{s_{2}}$ & $P_{f}$ & $P_{m}$ & $P_{c}$ \\
\hline$m$ & $f$ & $h_{1}$ & $h_{2}$ & $h_{1}$ & $h_{2}$ & $\left(h_{1}, h_{1}\right)$ \\
$s_{1}$ & $s_{1}$ & $h_{2}$ & $h_{1}$ & $h_{2}$ & $h_{1}$ & $\left(h_{1}, h_{2}\right)$ \\
$f$ & $m$ & & & & & $\left(h_{2}, h_{2}\right)$ \\
$s_{2}$ & $s_{2}$ & & & & & $\left(h_{2}, h_{1}\right)$ \\
\hline
\end{tabular}

Table 2.4: Violation of CPC under AER

We show that there is no stable matching at this preference profile. Assume for contradiction that $\mu$ is a stable matching at that profile. Now, we consider all possible cases of couple's matching and show that $\mu$ is blocked in each of those cases. 
- Suppose $\mu(c)=\left(h_{1}, h_{1}\right)$. Since $h_{1} P_{s_{1}} h_{2}$ and $s_{1} P_{h_{1}} f, \mu$ is blocked by $\left(h_{1}, s_{1}\right)$.

- Suppose $\mu(c)=\left(h_{1}, h_{2}\right)$. Since $\left(h_{1}, h_{1}\right) P_{c}\left(h_{1}, h_{2}\right)$ and $m P_{h_{1}} s_{1} P_{h_{1}} s_{2}, \mu$ is blocked by $\left(\left(h_{1}, h_{1}\right), c\right)$.

- Suppose $\mu(c)=\left(h_{2}, h_{2}\right)$. Since $\left(h_{1}, h_{1}\right) P_{c}\left(h_{2}, h_{2}\right), m P_{h_{1}} s_{1}$, and $f P_{h_{1}} s_{2}, \mu$ is blocked by $\left(\left(h_{1}, h_{1}\right), c\right)$.

- Suppose $\mu(c)=\left(h_{2}, h_{1}\right)$. Since $\left(h_{2}, h_{2}\right) P_{c}\left(h_{2}, h_{1}\right),\left(h_{1}, h_{1}\right) P_{c}\left(h_{2}, h_{1}\right), f P_{h_{1}} s_{2}$ and $m P_{h_{2}} s_{2}, \mu\left(s_{2}\right)=h_{1}$ implies $\left(\left(h_{1}, h_{1}\right), c\right)$ blocks $\mu$ and $\mu\left(s_{2}\right)=h_{2}$ implies $\left(\left(h_{2}, h_{2}\right), c\right)$ blocks $\mu$.

Therefore, there is no stable matching at the preference profile given in Table 2.4.

\subsection{Conclusion}

In this chapter, we have considered many-to-one matching problems between doctors and hospitals where doctors consist of some couples. First, we have considered the case where the hospitals have a common preference over individual doctors. We have shown that when a couple is allowed to violate responsiveness only for togetherness, a stable matching exists at every preference profile if and only if the lesser preferred member (according to the common preference of the hospitals) of the couple is ready to violate responsiveness to be together with the more preferred member. We have further provided necessary and sufficient condition for the existence of a stable matching at every preference profile when a couple is allowed to violate responsiveness arbitrarily.

Next, we have considered the case where hospitals need not have a common preference over individual doctors. We have shown that if couples' preferences satisfy the almost everywhere responsive property and hospitals' preferences satisfy common preference over couples property, then a stable matching exists at every preference profile.

An interesting open problem would be to consider the situation where (i) hospitals are partitioned based on geographical regions (i.e., hospitals in the same geographical region are in one partition) and (ii) couples' preferences violate responsiveness in order for them to be employed at hospitals that are located in the same region. It follows from Theorem 2.2 that a stable matching will exist in this model if hospitals 
preferences satisfy SCPI. However, SCPI need not be a necessary condition for the existence of stable matching in this case since there is some structure in the way couples' preference can violate responsiveness. We leave the problem of finding the exact necessary and sufficient condition for the existence of a stable matching in this model for future research. 
CHAPTER 3

\section{Stability in Matching with Couples having Responsive Preferences}

\subsection{Introduction ${ }^{1}$}

A huge literature has been developed on various market designs for finding an "optimal" matching procedure in a labour market. In many centralised labour markets, stability is an important condition for optimality. We have already seen that a matching is stable when there do not exist institution-individual pairs that are not matched to each other, but when being matched are better off compared to their present allocation.

Kelso and Crawford (1982), Roth (1985), Alkan and Gale (2003) and Hatfield and Milgrom (2004) show that a sufficient amount of substitutability can guarantee the

\footnotetext{
${ }^{1}$ Although some of the notations, definitions and explanations are already discussed in the first two chapters, we state them again for completeness.
} 
existence of a stable matching. These papers assume substitutability of preferences of the hospitals over sets of doctors. Later, Klaus and Klijn (2005) assume responsiveness of couples' preferences over ordered pairs of hospitals and show that the existence of a stable matching is guaranteed in such settings. Responsiveness means that unilateral improvements according to the preference of one couple member are beneficial for the couple.

As in previous chapters, we assume here that the institutions are hospitals and the individuals are doctors, where the subset of doctors includes couples. We assume that couples' preferences are responsive over the pair of hospitals and hospitals' preferences are responsive over the set of doctors.

Note that given the individual preferences of the couple members, more than one (joint) couple preference satisfies responsiveness. For example, suppose we have a couple $c=\{f, m\}$ and two hospitals $h_{1}$ and $h_{2}$ such that $f$ prefers $h_{1}$ to $h_{2}$ but $m$ prefers $h_{2}$ to $h_{1}$. Suppose further that $(x, y)$ denotes an allocation of this couple where $f$ is matched with $x$ and $m$ is matched with $y$. Then we know by responsiveness that $\left(h_{1}, h_{2}\right)$ is preferred to both $\left(h_{1}, h_{1}\right)$ and $\left(h_{2}, h_{2}\right)$ whereas, $\left(h_{1}, h_{1}\right)$ and $\left(h_{2}, h_{2}\right)$ are both preferred to $\left(h_{2}, h_{1}\right)$. However, responsiveness does not give us an ordering between the pairs $\left(h_{1}, h_{1}\right)$ and $\left(h_{2}, h_{2}\right)$. Thus there are different complete responsive couple preference extensions. Similarly, given a preference of a hospital over doctors, we can have more than one responsive preference over subsets of doctors.

Allowing for complete responsive preferences for both couples of doctors and hospitals is not innocuous, as in such situations stable matchings may fail to exist. On the other hand, allowing that the pairs $\left(h_{1}, h_{1}\right)$ and $\left(h_{2}, h_{2}\right)$ are comparable in the above mentioned example of couple preference seems to be natural. Similarly, it seems natural that hospitals admitting teams of doctors compare, for instance, two pairs of doctors, where in one pair, there is one $\mathrm{A}$ ranked doctor and one $\mathrm{C}$ ranked doctor, and in the other pair there are two B ranked doctors. As we will see, allowing for such comparisons does affect the stability of matchings. Also, it is worthwhile to mention that by a standard replication of hospitals up to their capacities to create a one-toone matching, these pairs of doctors are actually considered incomparable. This is because, for the hospital the $\mathrm{B}$ ranked doctor is better than the $\mathrm{C}$ ranked doctor but is worse than the A ranked doctor. Thus, replication implies that these two sets of doctors are incomparable. This is the main reason why results presented here differ from Klaus and Klijn (2005), who found stable matchings in every case of couples' responsive preferences. 
In this chapter, we formalize our idea of complete responsive preferences of hospitals and couples and study its consequences for the existence of a stable matching.

First, we show by means of an example that stable matchings may not exist at arbitrary responsive preference profiles. Then, we provide a condition on couples' preferences that is necessary and sufficient for guaranteeing the existence of a stable matching for every responsive extension of hospitals' preferences over sets of doctors. We call this condition extreme-altruism. We explain this condition by means of the following example. Suppose that there are $k$ hospitals $\left\{h_{1}, \ldots, h_{k}\right\}$ and a couple. Suppose further that $h_{i}$ is preferred to $h_{j}$ for all $1 \leq i<j \leq k$ by both members of the couple. Then, extreme-altruism says that, for any $1 \leq i<j \leq k$, the couple prefers the allocation where one member is matched with $h_{i}$ and the other is matched with $h_{k}$ compared to the allocation where both of them are matched with $h_{j}$.

Next, we provide conditions on hospitals' preferences that are necessary and sufficient for guaranteeing the existence of a stable matching for every responsive extension of couples' preferences. We call this condition aversion to couple diversity. We explain this condition by means of the following example. Consider a hospital $h$ with preference $P_{h}$ over individual doctors. Let $c=\{f, m\}$ be a couple and let $d, d^{\prime}$ be two other doctors (possibly another couple). Suppose that $f P_{h} d P_{h} d^{\prime} P_{h} m$. As we have discussed earlier, responsiveness does not put any restriction on $h$ 's preference over the relative ordering of the sets of doctors $\{f, m\}$ and $\left\{d, d^{\prime}\right\}$. A hospital is averse of diverse couples if in such situations it prefers $\left\{d, d^{\prime}\right\}$ to $\{f, m\}$. Loosely put, this property asserts that a hospital does not like to employ couples whose members are relatively more dissimilar (with respect to its preference over individual doctors).

Another important contribution in this chapter is that, not only we characterise profiles where stable matchings exist, but we also provide algorithms that produce a stable matching whenever that exists.

Thus, we feel that we provide a more complete picture on the issue of the existence of stable matchings when hospitals and couples have responsive preferences, and consequently complements the work of Klaus and Klijn (2005).

In the next section, we lay out the framework of our model and formally provide all the necessary definitions. We state and describe an algorithm used for matching doctors with hospitals, which we use throughout this chapter. In Section 3.3, we provide an example showing that the existence of a stable matching is not guaranteed at arbitrary responsive preferences profiles. In Section 3.4, we present conditions on couples' preferences that are necessary and sufficient for guaranteeing the existence 
of a stable matching for arbitrary responsive extensions hospitals' preferences, and in Section 3.5, we present conditions on hospitals' preferences that are necessary and sufficient for guaranteeing the existence of a stable matching for arbitrary responsive extensions of couples' preferences. We conclude with Section 3.6 where we explain the difference between our work and Klaus and Klijn (2005) by means of a formal example.

\subsection{The framework}

We consider many-to-one matchings between doctors and hospitals. We denote by $H$ a finite set of hospitals. Each hospital $h \in H$ has a finite capacity, denoted by $\kappa_{h}$.

We denote by $D$ a finite set of doctors. We assume that $D=M \cup F \cup S$ where $F, M, S$ are pairwise disjoint sets with $|M|=|F|$. Here, the doctors in $F$ and $M$ together form fixed couples. Also, the doctors in $S$ are those who are not part of any couple. We denote the set of couples by $C=\left\{\left\{f_{1}, m_{1}\right\},\left\{f_{2}, m_{2}\right\}, \ldots\right\}$ and a generic couple by $c=\{f, m\}$. We denote by $\lambda \notin H$, a dummy hospital which we use to represent a doctor being unemployed.

Throughout this chapter, we assume $|H| \geq 2,|D| \geq 4,|C| \geq 1$, and $\kappa_{h} \geq 2$ for all $h \in H$ and $\kappa_{\lambda}=|D|$.

For notational convenience, we do not always use braces for denoting singleton hospitals, doctors or couples.

\subsubsection{Matching}

Definition 3.1. A matching $\mu$ is a correspondence from $H \cup\{\lambda\}$ to $D$ such that for all $h \in H,|\mu(h)| \leq \kappa_{h}$. Moreover $\mu\left(h_{1}\right) \cap \mu\left(h_{2}\right)=\emptyset$ for any $h_{1}, h_{2} \in H$ with $h_{1} \neq h_{2}$.

For ease of notation, whenever $d \in \mu(h)$ for some $d \in D$ and $h \in H$, we write $\mu(d)=$ $h$. We say that a doctor is matched with $\lambda$ to mean that the doctor is unemployed. More formally, if $d \notin \mu(h)$ for all $h \in H$, then $\mu(d)=\lambda$. For a couple $c=\{f, m\} \in C$ and for hospitals $h, h^{\prime} \in H \cup\{\lambda\}$, we write $\mu(c)=\left(h, h^{\prime}\right)$ to mean $\mu(f)=h$ and $\mu(m)=h^{\prime}$. Further, for a hospital $h \in H$ and a matching $\mu$, we say $h$ has $\kappa_{h}-|\mu(h)|$ vacant positions at $\mu$. 


\subsubsection{Preferences}

In this section, we introduce the notion of preferences of doctors and hospitals, and present some restrictions on them.

For a set $X$, we denote by $\mathbb{L}(X)$ the set of linear orders on $X$, i.e., complete, reflexive, transitive, and antisymmetric binary relations over $X$. An element of $\mathbb{L}(X)$ is called a preference (over $X$ ). For any $i \in H \cup D \cup C, R_{i}$ denotes a preference of $i$ and $P_{i}$ denotes its strict part. Since a preference is antisymmetric $x R_{i} y$ implies either $x=y$ or $x P_{i} y$. We say $x$ is weakly preferred to $y$ to mean $x R_{i} y$, and $x$ is (strictly) preferred to $y$ to mean $x P_{i} y$. For $P_{i} \in \mathbb{L}(X)$ and $k \leq|X|$, we define the $k$-th ranked element in $P_{i}$, denoted by $r_{k}\left(P_{i}\right)$, as follows: $r_{k}\left(P_{i}\right)=x \in X$ if $\left|\left\{y \in X: y R_{i} x\right\}\right|=k$.

\section{Preferences of hospitals}

For any hospital $h \in H$, let $D_{h}$ be the set of acceptable doctors. A preference of hospital $h$, denoted by $\bar{P}_{h}$, is a linear order over $D_{h}$. Thus, $\bar{P}_{h} \in \mathbb{L}\left(D_{h}\right)$. A hospital prefers to have any doctor from this set of acceptable doctors, over having a vacant spot. Similarly, a hospital prefers to have a vacant spot to having doctors which do not belong to the set of acceptable doctors. We assume that the dummy hospital $\lambda$ finds all doctors acceptable. Thus, $D_{\lambda}=D$. Also, $\lambda$ is indifferent between all doctors.

For any hospital $h \in H$, a preference $\bar{P}_{h}$ over individual doctors is extended to a preference $P_{h}$ over feasible subsets of acceptable doctors $\left\{D^{\prime} \subseteq D_{h}:\left|D^{\prime}\right| \leq \kappa_{h}\right\}$.

Definition 3.2. We say $P_{h} \in \mathbb{L}\left(\left\{D^{\prime} \subseteq D_{h}:\left|D^{\prime}\right| \leq \kappa_{h}\right\}\right)$ is responsive if

(i) for all $D^{\prime} \subseteq D_{h}$ with $D^{\prime} \neq \emptyset$ and $\left|D^{\prime}\right| \leq \kappa_{h}, D^{\prime} P_{h} \emptyset$.

(ii) for all $d, d^{\prime} \in D_{h},\{d\} R_{h}\left\{d^{\prime}\right\}$ if and only if $d \bar{R}_{h} d^{\prime}$, and

(iii) for all $D^{\prime}, D^{\prime \prime} \subseteq D_{h}$ with $\left|D^{\prime}\right|<\kappa_{h},\left|D^{\prime \prime}\right|<\kappa_{h}$ and all $d \in D_{h} \backslash\left(D^{\prime} \cup D^{\prime \prime}\right),\left(D^{\prime} \cup\right.$ $\{d\}) P_{h}\left(D^{\prime \prime} \cup\{d\}\right)$ if and only if $D^{\prime} P_{h} D^{\prime \prime}$.

Having define $P_{h}$ over all feasible subsets of acceptable doctors, we extend this preference over the set of all feasible subsets of doctors $\left\{D^{\prime} \subseteq D:\left|D^{\prime}\right| \leq \kappa_{h}\right\}$.

Definition 3.3. For all $D^{\prime} \subseteq D$ such that $\left|D^{\prime}\right| \leq \kappa_{h}$ and $D^{\prime} \not \subset D_{h}$, we have $\emptyset P_{h} D^{\prime}$. 
For notational convenience, for any hospital $h \in H$, any couple $c=\{f, m\} \in C$ and any doctor $d \in D \backslash\{f, m\}, d P_{h} c$ means $d P_{h} f$ and $d P_{h} m$.

\section{Preferences of doctors and couples}

A preference of a doctor $d \in D$, denoted by $P_{d}$, is an element of $\mathbb{L}(H \cup\{\lambda\})$. A preference of a couple $c=\{f, m\} \in C$, denoted by $P_{c}$, is an element of $\mathbb{L}\left((H \cup\{\lambda\})^{2}\right)$. We call a preference of a couple responsive if a unilateral improvement in the position of one member of the couple is beneficial for the couple.

Definition 3.4. Let $c=\{f, m\} \in C$ be a couple. Let $P_{f}$ be a preference of $f$ and $P_{m}$ be a preference of $m$. A preference $P_{c} \in \mathbb{L}\left((H \cup\{\lambda\})^{2}\right)$ of the couple $c$ is called responsive (with respect to $P_{f}$ and $P_{m}$ ) if for all $h, h_{1}, h_{2} \in H \cup\{\lambda\}$, we have

(i) $\left(h_{1}, h\right) P_{c}\left(h_{2}, h\right)$ if and only if $h_{1} P_{f} h_{2}$, and

(ii) $\left(h, h_{1}\right) P_{c}\left(h, h_{2}\right)$ if and only if $h_{1} P_{m} h_{2}$.

For any $c=\{f, m\} \in C$, a responsive preference $P_{c}$ induces unique marginal preferences $P_{f}$ and $P_{m}$ for $f$ and $m$ respectively.

\section{Preference profiles and matching problems}

A preference profile is a collection of responsive preferences for all hospitals in $H$, all doctors in $D$ and all couples in $C$. Thus, a preference profile $P$ is a tuple of preferences $\left(\left\{P_{d}\right\}_{d \in D},\left\{P_{c}\right\}_{c \in C},\left\{P_{h}\right\}_{h \in H}\right)$, where for all $d \in D, c \in C$ and $h \in H, P_{d}$ is a preference of doctor $d, P_{c}$ is a responsive preference of couple $c$, and $P_{h}$ is a responsive preference of hospital $h$ over acceptable and feasible sets of doctors, respectively. Note that, for any hospital $h \in H, D_{h}$ is an inherent part of $P_{h}$. This means that a preference $P_{h}$ automatically specifies the acceptable set $D_{h}$ of the hospital.

A matching problem is a tuple consisting of a set of hospitals with corresponding capacities, a set of doctors with its partition into $F, M, S$, and a corresponding preference profile. 


\subsubsection{Stability}

There are different notions of stability based on different types of permissible blocking coalitions.

Let $\mu$ be a matching and $P$ be a preference profile. We say a hospital $h$ prefers to have a set of doctors $D^{\prime}$ (possibly empty) to a subset of doctors in $\mu(h)$ if there is $D^{\prime \prime} \subseteq \mu(h)$ with $D^{\prime} \cap D^{\prime \prime}=\emptyset$ such that $\left\{\left(\mu(h) \backslash D^{\prime \prime}\right) \cup D^{\prime}\right\} P_{h} \mu(h)$. Similarly, we say a doctor $d$ (or a couple $c$ ) prefers a hospital $h$ to $\mu(d)$ (or a pair of hospitals $\left(h, h^{\prime}\right)$ to $\mu(c))$ if $h P_{d} \mu(h)$ (or $\left(h, h^{\prime}\right) P_{c} \mu(c)$ ). Note that if a hospital prefers a set of doctors to its assignment at $\mu$, then by definition, that hospital is not matched with any of those doctors at $\mu$. Moreover, it could also be that $h$ has some unacceptable doctors $D^{\prime \prime}$ in $\mu(h)$, thus $h$ prefers $D^{\prime}=\emptyset$ to $\mu(h)$.

Similarly, if a doctor (or a couple) prefers a hospital (or a pair of hospitals) to its assignment at $\mu$, then that doctor (or at least one member of that couple) is not matched with the hospital (or the corresponding hospital) at $\mu$.

Now, we define the notion of blocking. Note that, since $\lambda$ is indifferent between all sets of doctors, and $D_{\lambda}=D$, thus $\lambda$ always prefers to have any doctor than not having that doctor. First, we introduce the notion of blocking between a hospital and a doctor in $S$.

Definition 3.5. Let $s \in S, h \in H \cup\{\lambda\}$ and let $\mu$ be a matching. Then $(h, s)$ blocks $\mu$ if $h$ prefers $s$ to $\mu(h)$ and s prefers $h$ to $\mu(s)$.

Next, we define the notion of blocking between a pair of hospitals and a couple.

Definition 3.6. Let $\mu$ be a matching and let $c=\{f, m\} \in C$ and $\left(h_{f}, h_{m}\right) \in$ $(H \cup\{\lambda\})^{2}$. Then, $\left(\left(h_{f}, h_{m}\right), c\right)$ blocks $\mu$ if c prefers $\left(h_{f}, h_{m}\right)$ to $\mu(c)$ and

(i) if $h_{f} \neq h_{m}$ and $\mu(f) \neq h_{f}$, then $h_{f}$ prefers $f$ to $\mu\left(h_{f}\right)$,

(ii) if $h_{f} \neq h_{m}$ and $\mu(m) \neq h_{m}$, then $h_{m}$ prefers $m$ to $\mu\left(h_{m}\right)$, and

(iii) if $h_{f}=h_{m}$, then $h_{f}$ prefers $\{f, m\}$ to $\mu\left(h_{f}\right)$.

An important thing to note about the notion of blocking here is that hospitals are myopic in the sense that when they remove a member of a couple, they do not consider the possibility of losing the other member of it. We consider this model as otherwise 
a farsighted notion of blocking would require a hospital to estimate the possibility of losing the 'other member' by checking whether there is really any hospital for that member to go (to be better off individually or by couple's preference). Such a notion will complicate the model considerably, and more importantly, doing so much of calculations in order to decide who to appoint or remove is practically impossible for big institutions like hospitals.

Thus, by allowing the notion of a hospital being interested in a couple, the blocking definition takes complementarity of a couple being accepted into account. However, the hospital does not take this into account while accepting single doctors at the cost of removing a member of the couple from the hospital. The asymmetry arising here is the main reason, why the results obtained in this chapter are different to the results obtained by choice function approach in many-to-many matchings. ${ }^{2}$

Whenever a matching $\mu$ is blocked by $\left(\left(h_{f}, \mu(m)\right), c\right)$ for some $c=\{f, m\} \in C$ and some $h_{f} \in H \cup\{\lambda\}$, for ease of presentation we say that $\mu$ is blocked by $\left(h_{f}, f\right)$. Similarly we say that $\mu$ is blocked by $\left(h_{m}, m\right)$ if $\mu$ is blocked by $\left(\left(\mu(f), h_{m}\right), c\right)$.

Our next remark follows from the responsiveness of couples' preferences and Definition 3.6.

Remark 3.1. Let $\mu$ be a matching and $c=\{f, m\}$ be a couple. Suppose for some $x \in$ $\{f, m\}$ and some hospital $h_{x} \in H$, we have $h_{x} P_{x} \mu(x)$ and $\left(\left(\mu\left(h_{x}\right) \backslash d\right) \cup\{x\}\right) P_{h_{x}} \mu\left(h_{x}\right)$ for some $d \in \mu\left(h_{x}\right) \backslash\{f, m\}$, then $\left(h_{x}, x\right)$ blocks $\mu$.

A matching is stable if it cannot be blocked by any blocking pair. More formally, we get the following definition.

Definition 3.7. A matching $\mu$ is stable, if

(i) for all $h \in H \cup \lambda$ and $s \in S$, $(h, s)$ does not block $\mu$,

(ii) for all $\left(h_{f}, h_{m}\right) \in(H \cup \lambda)^{2}$ and $c \in C,\left(\left(h_{f}, h_{m}\right), c\right)$ does not block $\mu$, and

(iii) for all $h \in H,(h, \emptyset)$ does not block $\mu(h)$, i.e., $h$ does not prefer $\emptyset$ to $\mu(h)$.

Now, we define the concept of individual rationality.

Definition 3.8. A matching $\mu$ is individually rational if

\footnotetext{
${ }^{2}$ See Konishi and Ünver (2006), Echenique and Oviedo (2006), Hatfield and Kojima (2010), for notions of stability in many-to-many matchings.
} 
(i) for all $s \in S, \mu(s) R_{s} \lambda$,

(ii) for all $c \in C, \mu(c) R_{c}(\lambda, \lambda)$, and

(iii) for all $h \in H$ and all $d \in \mu(h), d \in D_{h}$.

The next remark follows from the definition of stability.

Remark 3.2. Every stable matching is individually rational.

\subsubsection{Algorithm}

In this section we present a well-known algorithm called doctor proposing deferred acceptance algorithm (DPDA). This algorithm was introduced by Gale and Shapley(1962). ${ }^{3}$ Our proofs for the existence of stable matchings use a modification of DPDA. In what follows, we give a very short description of this algorithm. Take a profile $P$. Then, the DPDA algorithm at $P$ goes as follows.

$D P D A$ : In step 1 of the algorithm, all doctors simultaneously propose to their most preferred hospitals. Each hospital $h \in H$ provisionally accepts the most preferred doctors according to $P_{h}$. If a hospital receives more than $\kappa_{h}$ proposals, then it rejects all the doctors which do not belong to its $\kappa_{h}$ most preferred doctors. In any step $k$, the unmatched doctors propose to their most preferred hospital from the remaining set of hospitals who have not rejected them in any of the earlier steps. In any step of DPDA, since any hospital $h \in H$ accepts the most preferred collection of doctors according to $P_{h}$, it may reject some doctors that it had provisionally accepted earlier. Hospitals whose provisional list of accepted doctors is less than their maximum capacity can still add to their accepted list if they receive fresh proposals. Thus the algorithm terminates when each doctor is matched with some hospital or has been rejected by all acceptable hospitals.

Remark 3.3. Note that in DPDA, each individual doctor proposes according to his/her individual preference. Thus, couples' preferences do not play any role in this algorithm.

It is well-known that the outcome of DPDA is optimal for doctors. That is, some doctor is worse off at every other stable matching. Moreover, by responsiveness and the structure of DPDA, it follows that the outcome of DPDA is individually rational.

\footnotetext{
${ }^{3}$ See Knuth(1976), Gusfield and Irving(1989), Roth and Sotomayer(1990), Aldershof and Carducci(1996) for additional results on stable matching problem in two sided matching.
} 
The following remark follows directly from the definition of DPDA.

Remark 3.4. Let $\mu$ be the outcome of DPDA. Let $d \notin \mu(h)$ for some $d \in D$ and $h \in H$. Then, $h P_{d} \mu(d)$ implies $d^{\prime} P_{h} d$ for all $d^{\prime} \in \mu(h)$.

In the following lemmas, we show that the outcome of DPDA cannot be blocked by a hospital and a single doctor or by a pair of different hospitals and a couple. Some of these results are well known outcomes of DPDA, but we prove them nevertheless for the sake of completeness.

Lemma 3.1. The outcome of DPDA cannot be blocked by a pair $(h, s)$ for any $h \in H$ and any $s \in S$.

Proof. Let $\mu$ be the outcome of DPDA. Assume for contradiction that $(h, s)$ blocks $\mu$ for some $h \in H$ and some $s \in S$. Since the outcome of DPDA is individually rational, $s R_{h} \lambda$. Since $h P_{s} \mu(s)$, by the definition of DPDA and Remark 3.4, either $s$ has not proposed to $h$ during the DPDA or all the doctors in $\mu(h)$ are preferred to $s$ according to $P_{h}$. If $s$ has not proposed to $h$ during DPDA, then we have $\mu(s) P_{s} h$, a contradiction to the fact that $(h, s)$ blocks $\mu$. So, suppose $d P_{h} s$ for all $d \in \mu(h)$. Then, by responsiveness of hospitals' preferences, we have $\mu(h) P_{h}((\mu(h) \backslash\{d\}) \cup s)$ for all $d \in \mu(h)$, and consequently, hospital $h$ will not block with $s$. This completes the proof of the lemma.

Lemma 3.2. The outcome of DPDA cannot be blocked by $\left(\left(h_{1}, h_{2}\right), c\right)$ for any $h_{1}, h_{2} \in$ $H$ such that $h_{1} \neq h_{2}$ and for any $c \in C$.

Proof. Let $\mu$ be the outcome of DPDA. Assume for contradiction that $\mu$ is blocked by $\left(\left(h_{1}, h_{2}\right), c\right)$. Let $\mu(f)=h_{f}$ and $\mu(m)=h_{m}$. By the definition of a block, $\left(h_{1}, h_{2}\right) P_{c}\left(h_{f}, h_{m}\right)$.

Suppose $h_{f} R_{f} h_{1}$ and $h_{m} R_{m} h_{2}$. Since $\left(h_{1}, h_{2}\right) \neq\left(h_{f}, h_{m}\right)$, this means $\left(h_{f}, h_{m}\right) P_{c}\left(h_{1}, h_{2}\right)$, a contradiction. Now, suppose $h_{1} P_{f} h_{f}$ or $h_{2} P_{m} h_{m}$. Without loss of generality, assume $h_{2} P_{m} h_{m}$. Since the outcome of DPDA is individually rational, $h_{2} \neq \lambda$. Because $h_{2} P_{m} h_{m}$, by Remark 3.4, $m$ proposed to $h_{2}$ at some step of DPDA and got rejected. Since $h_{1} \neq h_{2}$, by Lemma 3.1, we have $\mu\left(h_{2}\right) P_{h_{2}}\left(\left(\mu\left(h_{2}\right) \backslash\{d\}\right) \cup m\right)$ for all $d \in \mu\left(h_{2}\right)$. However, this contradicts the definition of a block.

In what follows, we give a lemma which shows that the outcome of DPDA cannot be blocked by a pair of dummy hospitals and a couple. 
Lemma 3.3. The outcome of DPDA cannot be blocked by $((\lambda, \lambda), c)$ for any $c \in C$.

Proof. Let $\mu$ be the outcome of DPDA. Assume for contradiction that $\mu$ is blocked by $((\lambda, \lambda), c) . \quad \operatorname{Let} \mu(f)=h_{f}$ and $\mu(m)=h_{m} . \quad$ By the definition of block, $(\lambda, \lambda) P_{c}\left(h_{f}, h_{m}\right)$. By responsiveness, this means $\lambda P_{x} h_{x}$ for some $x \in\{f, m\}$. However, by the definition of DPDA, $\lambda P_{x} h_{x}$ means $x$ proposed to $\lambda$ before proposing to $h_{x}$ and got rejected. This is contradiction as, by our assumption, $\lambda$ cannot reject a doctor.

In the following lemmas, we give conditions when an outcome of DPDA cannot be blocked by a pair of same hospitals and a couple.

Lemma 3.4. Suppose $\mu$ is an outcome of DPDA. Then for any $h \in H$ and any $c=$ $\{f, m\} \in C$,

(i) $f P_{h} m$ and $h P_{f} \mu(f)$ implies $((h, h), c)$ cannot block $\mu$, and

(ii) $m P_{h} f$ and $h P_{m} \mu(m)$ implies $((h, h), c)$ cannot block $\mu$.

Proof. Assume without loss of generality that $f P_{h} m$ and $h P_{f} \mu(f)$. By the definition of DPDA, $f$ proposed to $h$ and got rejected. By Remark 3.4, $d P_{h} f$ for all $d \in \mu(h)$. Because $f P_{h} m,\left\{d, d^{\prime}\right\} P_{h}\{f, m\}$ for all $d, d^{\prime} \in \mu(h)$. This means $h$ cannot block $\mu$ with $c$. This completes the proof.

Lemma 3.5. Suppose $\mu$ is an outcome of DPDA. Then for any $h \in H$ and any $c=$ $\{f, m\} \in C,((h, h), c)$ cannot block $\mu$ if $h=\mu(x)$ for some $x \in\{f, m\}$

Proof. Without loss of generality, let $h=\mu(f)$. Let $((h, h), c)$ block $\mu$.

By the definition of a block, $(h, h) P_{c}(\mu(f), \mu(m))$. Thus, by responsiveness of couples' preferences, $h P_{m}^{0} h_{m}$. By the definition of DPDA, this means $m$ proposed to $h$ and got rejected. Since $f \in \mu(h)$, by Lemma 3.1 and the definition of block, we have $\mu(h) P_{h}((\mu(h) \backslash\{d\}) \cup m)$ for all $d \in \mu(h)$. This means $h$ will not block $\mu$ with $c$, which is a contradiction.

Remark 3.5. If a doctor $d \in D$ and hospital $h \in H$ are each other's top ranked alternative, then trivially for a stable match, they must be matched to each other. 


\subsection{Stable matching is not guaranteed at arbitrary re- sponsive profiles}

In this section, we show by means of two examples that existence of a stable matching is not guaranteed at arbitrary responsive preference profiles. The two examples are slightly different to suit the subsequent sections.

Example 3.1. Let $H=\left\{h_{1}, h_{2}, h_{3}\right\}, \kappa_{h}=2$ for all $h \in\left\{h_{1}, h_{2}, h_{3}\right\}$, and $D=$ $\left\{d_{1}, d_{2}, d_{3}, d_{4}, f, m\right\}$ where $c=\{f, m\}$ is a couple.

Suppose $r_{1}\left(P_{d_{1}}\right)=h_{2}$ and $r_{2}\left(P_{d_{1}}\right)=r_{1}\left(P_{d_{2}}\right)=h_{1}$. Further, $r_{1}\left(P_{d_{3}}\right)=h_{2}$ and $r_{1}\left(P_{d_{4}}\right)=$ $h_{3}$. For the couple, suppose $h_{2} P_{f} h_{1}, h_{2} P_{f} h_{3}$ and $h_{1} P_{m} h_{3}$ but $\left(h_{1}, h_{1}\right) P_{c}\left(h_{2}, h_{3}\right)$. Finally $h P_{x} \lambda$ for all $x \in\{f, m\}$ and all $h \in H$.

The above mentioned preferences along with the preferences of all the hospitals over individuals are given in Table 3.1. The preference of all hospitals over pairs of doctors can be any responsive preference over pairs of doctors. However $\{f, m\} P_{h_{1}}\left\{d_{1}, d_{2}\right\}$.

The couple's preference over pairs of hospitals, where one member is matched and the other one is unmatched are assumed to be responsive and ranked below the pairs of hospitals.

\begin{tabular}{cccccccccc}
\hline$P_{d_{1}}$ & $P_{d_{2}}$ & $P_{d_{3}}$ & $P_{d_{4}}$ & $P_{f}$ & $P_{m}$ & $P_{c}$ & $\bar{P}_{h_{1}}$ & $\bar{P}_{h_{2}}$ & $\bar{P}_{h_{3}}$ \\
\hline$h_{2}$ & $h_{1}$ & $h_{2}$ & $h_{3}$ & $\vdots$ & $\vdots$ & $\vdots$ & $d_{3}$ & $d_{3}$ & $d_{4}$ \\
$h_{1}$ & $\vdots$ & $\vdots$ & $\vdots$ & $h_{2}$ & $h_{1}$ & $\left(h_{1}, h_{1}\right)$ & $d_{4}$ & $d_{4}$ & $d_{3}$ \\
$\vdots$ & & & & $\vdots$ & $\vdots$ & $\vdots$ & $f$ & $f$ & $d_{1}$ \\
& & & & $h_{1}$ & $h_{3}$ & $\left(h_{2}, h_{3}\right)$ & $d_{1}$ & $d_{1}$ & $f$ \\
& & & & $\vdots$ & $\vdots$ & $\vdots$ & $d_{2}$ & $m$ & $m$ \\
& & & & & & & $m$ & $d_{2}$ & $d_{2}$ \\
\hline
\end{tabular}

Table 3.1: No Stable Matching under Responsive Preferences (Ex. 1)

The couple and $h_{1}$ have responsive preferences. In what follows, we argue that there is no stable matching for the preference profile given in Table 3.1.

Assume for contradiction, that there exists a stable matching $\mu$ for the given preference profile. By Remark 3.5, it must be that $\mu\left(d_{3}\right)=h_{2}$ and $\mu\left(d_{4}\right)=h_{3}$, as the doctors 
and hospitals are each other's top ranked alternative. Thus, there is potentially only one vacancy to be filled in $h_{2}$ and $h_{3}$ respectively.

Particularly, it is not possible for the couple to be matched to $\left(h_{2}, h_{2}\right)$ and $\left(h_{3}, h_{3}\right)$ for a stable matching. Also, since the couple prefers to be matched with any two hospitals than having at least one member of the couple unmatched and we have sufficiently many number of vacancies in all the three hospitals, thus, $\{\mu(f), \mu(m)\} \subseteq$ $\left\{h_{1}, h_{2}, h_{3}\right\}$.

Now we look at the following allocations of the couple in $\mu$.

(i) Suppose $\mu(c)=\left(h_{2}, h_{1}\right)$.

Note that $f P_{h_{2}} d_{1}$. Since $d_{i} \bar{P}_{h_{1}} m$ for $i \in\{1,2\}, r_{2}\left(P_{d_{1}}\right)=r_{1}\left(P_{d_{2}}\right)=h_{1}$. So, $\mu\left(h_{2}\right)=\left\{d_{3}, f\right\}$. As $\kappa_{h_{1}}=2$, therefore $d_{i} \notin \mu\left(h_{1}\right)$ for some $i$. Thus, stability of $\mu$ implies that $\left(h_{1}, d_{i}\right)$ blocks $\mu$.

(ii) Suppose $\mu(c)=\left(h_{1}, h_{1}\right)$.

Then $\left(h_{1}, d_{2}\right)$ blocks $\mu$.

(iii) Suppose $\mu(c)=\left(h_{2}, h_{3}\right)$.

Since $\{f, m\} P_{h_{1}}\left\{d_{1}, d_{2}\right\}$ and $\left(h_{1}, h_{1}\right) P_{c}\left(h_{2}, h_{3}\right),\left(\left(h_{1}, h_{1}\right), c\right)$ blocks $\mu$.

(iv) Suppose $\mu(c)=\left(h_{1}, h_{3}\right)$.

Since $h_{2} P_{f} h_{1}$, responsiveness implies $\left(h_{2}, h_{3}\right) P_{c}\left(h_{1}, h_{3}\right)$. This together with the fact that $f \bar{P}_{h_{2}} d_{1} \bar{P}_{h_{2}} d_{2}$ implies $\left(\left(h_{2}, h_{3}\right), c\right)$ blocks $\mu$.

(v) Suppose $\mu(c)=\left(h_{3}, h_{1}\right)$.

Since $h_{2} P_{f} h_{3}$, responsiveness implies $\left(h_{2}, h_{1}\right) P_{c}\left(h_{3}, h_{1}\right)$. This together with the fact that $f \bar{P}_{h_{2}} d_{1} \bar{P}_{h_{2}} d_{2}$ implies $\left(\left(h_{3}, h_{1}\right), c\right)$ blocks $\mu$.

(vi) Suppose $\mu(c)=\left(h, h_{2}\right)$ for some $h \in\left\{h_{1}, h_{3}\right\}$.

Thus, $d_{1} \notin \mu\left(h_{2}\right)$. Since $r_{1}\left(P_{d_{1}}\right)=h_{2}$ and $d_{1} P_{h_{2}} m$, stability of $\mu$ implies that $\left(h_{2}, d_{1}\right)$ blocks $\mu$.

Cases (i)-(vi) together are exhaustive. Thus, it follows that there is no stable matching for the given preference profile in Table 3.1.

Note that, the arguments of this example can also be used to give an example with no stable matching when we consider $h_{2}$ and $h_{3}$ as the same hospital. Thus, we just consider two vacancies for both the hospitals while excluding $d_{3}$ and $d_{4}$ from 
the set of doctors. It follows from the above argument that there does not exist a stable matching when $\left(h_{1}, h_{1}\right) P_{c}\left(h_{2}, h_{2}\right)$ with $h_{2} P_{f} h_{1}, h_{1} P_{m} h_{2}$ and the preferences of hospitals over $\left\{f, m, d_{1}, d_{2}\right\}$ as given by $\bar{P}_{h_{1}}$ and $\bar{P}_{h_{2}}$.

Example 3.2. Let $H=\left\{h_{1}\right\}, \kappa_{h_{1}}=2$ and $D=\left\{d_{1}, d_{2}, f, m\right\}$ where $c=\{f, m\}$ is a couple.

The preference of the hospital over individuals and pairs of individuals, preference of individual doctors and the couple preferences are given in Table 3.2.

The preference of $h_{1}$ over the pairs of doctors where at least one position is vacant is assumed to ranked below the shown pairs.

\begin{tabular}{ccccccc}
\hline $\bar{P}_{h_{1}}$ & $P_{d_{1}}$ & $P_{d_{2}}$ & $P_{f}$ & $P_{m}$ & $P_{c}$ & $P_{h_{1}}$ \\
\hline$f$ & $h_{1}$ & $h_{1}$ & $\lambda$ & $h_{1}$ & $\left(\lambda, h_{1}\right)$ & $\left\{f, d_{1}\right\}$ \\
$d_{1}$ & $\lambda$ & $\lambda$ & $h_{1}$ & $\lambda$ & $\left(h_{1}, h_{1}\right)$ & $\left\{f, d_{2}\right\}$ \\
$d_{2}$ & & & & & $(\lambda, \lambda)$ & $\{f, m\}$ \\
$m$ & & & & & $\left(h_{1}, \lambda\right)$ & $\left\{d_{1}, d_{2}\right\}$ \\
& & & & & & $\left\{d_{1}, m\right\}$ \\
& & & & & & $\left\{d_{2}, m\right\}$ \\
\hline
\end{tabular}

Table 3.2: No Stable Matching under Responsive Preferences (Ex. 2)

Note that the couple and $h_{1}$ have responsive preferences In what follows, we argue that there is no stable matching for the preference profile given in Table 3.2.

Assume for contradiction, that there exists a stable matching $\mu$ for the given preference profile. We look at the following allocations of the couple in $\mu$.

(i) Suppose $\mu(c)=\left(\lambda, h_{1}\right)$.

Since $d_{i} \bar{P}_{h_{1}} m, r_{1}\left(d_{i}\right)=h_{1}$ for $i \in\{1,2\}$, and $\kappa_{h_{1}}=2$, therefore $d_{i} \notin \mu\left(h_{1}\right)$ for some $i$. Thus, stability of $\mu$ implies that $\left(h_{1}, d_{i}\right)$ blocks $\mu$.

(ii) Suppose $\mu(c)=(\lambda, \lambda)$.

Since $\{f, m\} P_{h_{1}}\left\{d_{1}, d_{2}\right\}$ and $\left(h_{1}, h_{1}\right) P_{c}(\lambda, \lambda),\left(\left(h_{1}, h_{1}\right), c\right)$ blocks $\mu$.

(iii) Suppose $\mu(c)=\left(h_{1}, h\right)$ for some $h \in\left\{h_{1}, \lambda\right\}$.

Since $r_{1}\left(P_{f}\right)=\lambda$, it follows by responsiveness that $((\lambda, h), c)$ blocks $\mu$.

Since cases (i)-(iii) are exhaustive, it follows that there is no stable matching for the given preference profile in Table 3.2. 


\subsection{Conditions on couples' preferences for stability}

In view of Examples 3.1 and 3.2, we look for necessary and sufficient conditions on couples' preferences that guarantee the existence of a stable matching for every profile.

Let $P_{C}^{0}=\left(\left\{P_{c}^{0}\right\}_{c \in C}\right)$ be a given profile of preferences of the couples. Thus, for any $c=\{f, m\} \in C$, and a given couple preference $P_{c}^{0}, P_{f}^{0}$ and $P_{m}^{0}$ denote the individual preferences of $f$ and $m$ respectively. Since preferences of couples are responsive, a couple preference $P_{c}^{0}$ uniquely determines the individual preferences $P_{f}^{0}$ and $P_{m}^{0}$ of the members of the couple. In what follows, we present a condition on $P_{C}^{0}$ called extreme-altruism.

Definition 3.9. A profile of couple preferences $P_{C}^{0}$ is said to satisfy extreme-altruism if for all $c=\{f, m\} \in C$, all $h \in H$ and all $h^{\prime}, h^{\prime \prime} \in H \cup\{\lambda\}$ :

(i) $h^{\prime} P_{f}^{0} h, h^{\prime} R_{f}^{0} h^{\prime \prime}, h^{\prime \prime} R_{m}^{0} \lambda$ and $\kappa_{h} \leq|D|-2$ imply $\left(h^{\prime}, h^{\prime \prime}\right) P_{c}^{0}(h, h)$, and

(ii) $h^{\prime} P_{m}^{0} h, h^{\prime} R_{m}^{0} h^{\prime \prime}, h^{\prime \prime} R_{f}^{0} \lambda$ and $\kappa_{h} \leq|D|-2$ imply $\left(h^{\prime \prime}, h^{\prime}\right) P_{c}^{0}(h, h)$.

For illustration of extreme-altruism, consider a couple $c=\{f, m\}$ and hospitals $h \in H$ and $h^{\prime}, h^{\prime \prime} \in H \cup\{\lambda\}$. Suppose $f$ strictly prefers $h^{\prime}$ to $h$ and weakly prefers $h^{\prime}$ to $h^{\prime \prime}$, and $m$ weakly prefers $h^{\prime \prime}$ to $\lambda$. Then extreme-altruism says that, if $h$ does not have sufficiently large capacity, then $c$ ranks $\left(h^{\prime}, h^{\prime \prime}\right)$ above $(h, h)$. Note here, that if $h$ has a sufficiently large capacity, then we would not require any restriction on couples' preferences, simply because the hospital $h$ will have enough vacancies to accommodate at least one member of the couple, thereby removing the possibility of the couple to block with the hospital.

Out of two allocations of a couple, one where both the members are allocated at the same hospital and the other where one member moves to a better hospital (according to his/her individual preference), the couple prefers the latter to the former. For instance, if the hospital $h^{\prime}$ gives a substantially better salary compared to $h$. Then the couple would rather prefer on member to be at $h^{\prime}$ than both the members of the couple to be at $h$.

Our next lemma shows that extreme-altruism and responsiveness together imply that if two hospitals are acceptable for both members of a couple, then the ranking of those two hospitals by each member of the couple is always the same. That is, if $h_{1}$ 
and $h_{2}$ are both acceptable by each member of a couple $\{f, m\}$, then $f$ and $m$ will have same ranking over $h_{1}$ and $h_{2}$.

Lemma 3.6. Let $P_{C}^{0}$ be a profile of couple preferences satisfying extreme-altruism. Then, for any $c=\{f, m\}$ and any $h_{1}, h_{2} \in H$ such that $h P_{x}^{0} \lambda$ for all $x \in\{f, m\}$ and all $h \in\left\{h_{1}, h_{2}\right\}$, we have $h_{1} P_{f}^{0} h_{2}$ if and only if $h_{1} P_{m}^{0} h_{2}$.

Proof. Let $P_{C}^{0}$ be a profile of couple preferences satisfying extreme-altruism. Consider a couple $c=\{f, m\}$ with preference $P_{c}^{0}$ and consider two hospitals $h_{1}, h_{2} \in H$. Assume for contradiction $h_{1} P_{f}^{0} h_{2} P_{f}^{0} \lambda$ and $h_{2} P_{m}^{0} h_{1} P_{m}^{0} \lambda$. By responsiveness and Condition (i) of the definition of extreme-altruism, we have $\left(h_{1}, \lambda\right) P_{c}^{0}\left(h_{2}, h_{2}\right) P_{c}^{0}\left(\lambda, h_{2}\right)$. Again, responsiveness and Condition (ii) of extremealtruism implies $\left(\lambda, h_{2}\right) P_{c}^{0}\left(h_{1}, h_{1}\right) P_{c}^{0}\left(h_{1}, \lambda\right)$. However, this is a contradiction.

Our next corollary follows directly from Lemma 3.6. It says the following. Suppose $P_{C}^{0}$ satisfies the extreme-altruism. Consider a couple. Suppose that every hospital is acceptable for each member of the couple. Then, the members of the couple have the same individual preference over $H$.

Corollary 3.1. Let $P_{C}^{0}$ be a profile of couple preferences satisfying extreme-altruism. Let $c=\{f, m\} \in C$ be a couple such that $h P_{f}^{0} \lambda$ and $h P_{m}^{0} \lambda$ for all $h \in H$. Then $P_{m}^{0}=P_{f}^{0}$.

For a profile of preferences $P_{C}^{0}$ of the couples, an extension of $P_{C}^{0}$ is defined as a preference profile $P=\left(\left\{P_{d}\right\}_{d \in D},\left\{P_{c}\right\}_{c \in C},\left\{P_{h}\right\}_{h \in H}\right)$ such that $P_{c}=P_{c}^{0}$ for all $c \in C$.

The following theorem says that extreme-altruism of $P_{C}^{0}$ is necessary and sufficient for the existence of a stable matching for every extension of $P_{C}^{0}$.

Theorem 3.1. $\quad$ (i) If $P_{C}^{0}$ satisfies extreme-altruism, then a stable matching exists for any extension of $P_{C}^{0}$.

(ii) If $P_{C}^{0}$ does not satisfy extreme-altruism for all $h \in H$, then there exists an extension of $P_{C}^{0}$ with no stable matching.

Part (i). Consider a preference profile $P$ that is an arbitrary extension of $P_{C}^{0}$ where $P_{C}^{0}$ satisfies extreme-altruism. We show that the DPDA where each member of each couple proposes according to his/her individual preference gives a stable matching for $P$. 
Let $\mu$ be the outcome. Suppose $\mu$ is not stable at $P$. Since DPDA is individually rational, Lemma 3.1, Lemma 3.2 and Lemma 3.3 imply that $\mu$ is blocked by $((h, h), c)$ for some $h \in H$ and some $c=\{f, m\} \in C$. Let $\mu(f)=h_{f}$ and $\mu(m)=h_{m}$. Thus $(h, h) P_{c}^{0}\left(h_{f}, h_{m}\right)$.

Assume without loss of generality that $f P_{h} m$. By Lemma 3.4 and Lemma 3.5, we know that $h_{f} P_{f}^{0} h$. Thus, by responsiveness, we must have $h P_{m}^{0} h_{m}$. Suppose $\kappa_{h}>$ $|D|-2$. Then $\{f, m\} \notin \mu(h)$ implies $|\mu(h)| \leq|D|-2<\kappa_{h}$. But since $h P_{m}^{0} h_{m}, m$ proposed to $h$ at an earlier step of DPDA and got rejected even when $h$ had a vacancy. Thus $m \notin D_{h}$ which implies that $((h, h), c)$ cannot block $\mu$. Thus $\kappa_{h} \leq|D|-2$.

By the definition of DPDA, we have $h_{f} R_{f}^{0} \lambda$ and $h_{m} R_{m}^{0} \lambda$. Also, we know that $x$ is weakly preferred to itself. This together with extreme-altruism implies $\left(h_{f}, \lambda\right) P_{c}^{0}(h, h) P_{c}^{0}\left(h_{f}, h_{m}\right)$.

This contradicts the fact that $h_{m} R_{m}^{0} \lambda$. Thus $\left(h_{f}, h_{m}\right) R_{c}^{0}\left(h_{f}, \lambda\right) P_{c}^{0}(h, h)$ which contradicts that $((h, h), c)$ blocks $\mu$. This completes the proof of part (i) of Theorem 3.1.

[Part (ii)] Suppose $P_{C}^{0}$ does not satisfy extreme-altruism. We show that there exists an extension of $P_{C}^{0}$ with no stable matching.

Since $P_{C}^{0}$ does not satisfy extreme-altruism, there is a couple $c=\{f, m\}$, a hospital $h$ such that $\kappa_{h} \leq|D|-2$ and hospitals $h_{1}, h_{2} \in H \cup\{\lambda\} \backslash\{h\}$ such that:

(i) either, $h_{1} P_{f}^{0} h, h_{1} R_{f}^{0} h_{2}$ and $h_{2} R_{m}^{0} \lambda$, but $(h, h) P_{c}^{0}\left(h_{1}, h_{2}\right)$,

(ii) or, $h_{1} P_{m}^{0} h, h_{1} R_{m}^{0} h_{2}$ and $h_{2} R_{f}^{0} \lambda$, but $(h, h) P_{c}^{0}\left(h_{2}, h_{1}\right)$.

Assume without loss of generality that (i) holds.

By responsiveness, $h_{1} P_{f}^{0} h$ and $(h, h) P_{c}^{0}\left(h_{1}, h_{2}\right)$, implies $h P_{m}^{0} h_{2}$. Consider a preference profile $P$ such that

1. for all $h^{\prime} \in H \backslash\left\{h, h_{1}, h_{2}\right\}$, either $\mid\left\{d: d P_{h} c\right.$ and $\left.r_{1}\left(P_{d}\right)=h\right\} \mid=\kappa_{h^{\prime}}$ or $f, m \notin D_{h^{\prime}}$,

2. there are doctors $d_{1}, d_{2} \in D \backslash\{f, m\}$ such that $f P_{h} d_{1} P_{h} d_{2} P_{h} m$ and $\{f, m\} P_{h}\left\{d_{1}, d_{2}\right\}$

3. $\mid\left\{d: d P_{h} f\right.$ and $\left.r_{1}\left(P_{d}\right)=h\right\} \mid=\kappa_{h}-2$. This is possible since $\kappa_{h} \leq|D|-2$ implies $\kappa_{h}-2 \leq|D|-4$, 
4. either $f P_{h_{1}} d_{1} P_{h_{1}} m$ and $\mid\left\{d: d P_{h_{1}} f\right.$ and $\left.r_{1}\left(P_{d}\right)=h_{1}\right\} \mid=\kappa_{h_{1}}-1$, or $r_{1}\left(P_{h_{1}}\right)=f$, $r_{2}\left(P_{h_{1}}\right)=d_{1}$ and $m \notin D_{h_{1}}$,

5. either $\mid\left\{d: d P_{h_{2}} c\right.$ and $\left.r_{1}\left(P_{d}\right)=h_{2}\right\} \mid=\kappa_{h_{2}}-1$ or $f \notin D_{h_{2}}$,

6. $r_{1}\left(P_{d_{1}}\right)=h_{1}$ and $r_{2}\left(P_{d_{1}}\right)=r_{1}\left(P_{d_{2}}\right)=h$.

But it trivially follows from this preference profile that for a stable matching $\mu, h^{\prime} \notin$ $\{\mu(f), \mu(m)\}$ for $h^{\prime} \notin\left\{h, h_{1}, h_{2}\right\}$. Also, it is not possible that $\mu(c)=\left(h_{i}, h_{i}\right)$ for $i \in\{1,2\}$. Thus, by our construction, a stable matching exists for this matching problem if and only if there is a stable matching for Example 3.1. However, since there does not exist a stable matching for Example 3.1, thus, we do not have a stable matching for $P$. This completes the proof of part (ii) of Theorem 3.1.

From the above example, it is clear that even if all hospitals view all the doctors as acceptable, violation of extreme-altruism can lead to a preference profile with no stable matching, if we have enough doctors to fulfil the capacity constraints as given by points (i),(ii), (iv) and (v). Thus we get the following corollary.

Corollary 3.2. Suppose $D_{h}=D$ for all $h \in H$. Moreover, $\sum_{h \in H} \kappa_{h}=|D|$, then:

(i) If $P_{C}^{0}$ satisfies extreme-altruism, then a stable matching exists for any extension of $P_{C}^{0}$.

(ii) If $P_{C}^{0}$ does not satisfy extreme-altruism for all $h \in H$, then there exists an extension of $P_{C}^{0}$ with no stable matching.

\subsection{Conditions on hospitals' preferences for stability}

In Section 3.4, we have discussed a necessary and sufficient condition on couples' preferences that guarantees the existence of a stable matching for every collection of preferences of the hospitals. In this section, we look at the other side of the problem, that is, we look for necessary and sufficient condition on hospitals' preferences so that a stable matching exists for every collection of preferences of the doctors (both individuals and couples).

Let $P_{H}^{0}=\left(\left\{P_{h}^{0}\right\}_{h \in H}\right)$ be a given profile of preferences of the hospitals. In what follows, we introduce the aversion to couple diversity property. 
Definition 3.10. A profile of hospital preferences $P_{H}^{0}$ is said to have aversion to couple diversity if for all $h \in H$, all $c=\{f, m\}$ and all $d_{1}, d_{2} \in D$ with $f, m \in D_{h}$ such that either (i) $f P_{h}^{0} d_{1} P_{h}^{0} d_{2} P_{h}^{0} m$ and $\left|\left\{d: d P_{h}^{0} m\right\}\right|>\kappa_{h}$, or (ii) $m P_{h}^{0} d_{1} P_{h}^{0} d_{2} P_{h}^{0} f$ and $\left|\left\{d: d P_{h}^{0} f\right\}\right|>\kappa_{h}$, we have $\left\{d_{1}, d_{2}\right\} P_{h}^{0}\{f, m\}$.

Consider a hospital $h$ with a preference $P_{h}$ over acceptable and feasible sets of doctors. Take a couple $c=\{f, m\}$ such that both $f$ and $m$ are acceptable for $h$ but at least one of them is not amongst the top- $\kappa_{h}$ doctors according to the restriction of $P_{h}$ over individual doctors. Suppose that there are two doctors $d_{1}, d_{2}$ who are ranked inbetween $f$ and $m$ according to $P_{h}$. Then, aversion to couple diversity says that the set $\left\{d_{1}, d_{2}\right\}$ must be preferred to the couple $c$ according to $P_{h}$. Note here that if both the members of the couple are in the top- $\kappa_{h}$ doctors according to the restriction of $P_{h}$ over individual doctors, then we do not need this condition as for a stable matching, the couple will always be a part of $h$.

So, in other words, whenever a hospital compares a couple and another pair of doctors over which responsiveness does not induce the comparison, the hospital prefers the couple only if at most one doctor from the other pair ranks in-between the members of the couple. Thus, a hospital has aversion to couple diversity if it does not like to employ a couple whose members have relatively more dissimilar ranking in its preference.

It is important to note here that the diversity aversion just applies to couples and not single doctors as two single doctors can not apply to a hospital together and block a matching. On the other hand, we can encounter a situation where a couple applies to a hospital such that a member of a couple is individually worse off but the couple is better off as a whole.

For a profile of preferences $P_{H}^{0}$ of the hospitals, an extension of $P_{H}^{0}$ is defined as a preference profile $P=\left(\left\{P_{d}\right\}_{d \in D},\left\{P_{c}\right\}_{c \in C},\left\{P_{h}\right\}_{h \in H}\right)$ such that $P_{h}=P_{h}^{0}$ for all $h \in H$.

Our next theorem says that the aversion to couple diversity of $P_{H}^{0}$ is necessary and sufficient for the existence of a stable matching for every extension of $P_{H}^{0}$.

Theorem 3.2. (i) If $P_{H}^{0}$ satisfies aversion to couple diversity property, then a stable matching exists at every extension of $P_{H}^{0}$.

(ii) If $P_{H}^{0}$ does not satisfy aversion to couple diversity property, then there exists an extension of $P_{H}^{0}$ with no stable matching. 
Part (i). Consider a preference profile $P$ that is an arbitrary extension of $P_{H}^{0}$ where $P_{H}^{0}$ satisfies aversion to couple diversity. We show that the DPDA where each member of each couple proposes according to his/her individual preference gives a stable matching for $P$.

Let $\mu$ be the outcome. Suppose $\mu$ is not stable at $P$. Since DPDA is individually rational, Lemma 3.1, Lemma 3.2 and Lemma 3.3 imply that $\mu$ is blocked by $((h, h), c)$ for some $h \in H$ and some $c=\{f, m\} \in C$. Let $\mu(f)=h_{f}$ and $\mu(m)=h_{m}$. Thus $(h, h) P_{c}^{0}\left(h_{f}, h_{m}\right)$.

Assume without loss of generality that $f P_{h} m$. If $m \notin D_{h}$, then $((h, h), c)$ cannot block $\mu$ as it violates individual rationality. Thus $m P_{h}^{0} \lambda$. By Lemma 3.4 and Lemma 3.5, we know that $h_{f} P_{f}^{0} h$. Thus, responsiveness implies $h P_{m} h_{m}$.

It follows that, before applying to $h_{m}, m$ applied to $h$ and got rejected. Therefore, $\mid\left\{d: d P_{h}^{0} m\right.$ and $\left.d \in \mu(h)\right\} \mid=\kappa_{h}$. Since $f P_{h}^{0} m$ and $f \notin \mu(h)$, we have $\left|\left\{d: d P_{h}^{0} f\right\}\right|>$ $\kappa_{h}$. This, together with aversion to couple diversity, implies that $\left\{d, d^{\prime}\right\} P_{h}^{0}\{f, m\}$ for all $d, d^{\prime} \in \mu(h)$, which is a contradiction to the fact that $((h, h), c)$ blocks $\mu$. This completes the proof of part (i) of Theorem 3.2.

[Part (ii)] Suppose $P_{H}^{0}$ does not satisfy the aversion to couple diversity property. We show that there is an extension of $P_{H}^{0}$ with no stable matching.

Since $P_{H}^{0}$ does not satisfy the aversion to couple diversity property, we have $h \in H$, $c=\{f, m\} \in C$ and $d_{1}, d_{2} \in D$ with $f, m \in D_{h}$ such that $\{f, m\} P_{h}^{0}\left\{d_{1}, d_{2}\right\}$ and either (i) $f P_{h}^{0} d_{1} P_{h}^{0} d_{2} P_{h}^{0} m$ and $\left|\left\{d: d P_{h}^{0} m\right\}\right|>\kappa_{h}$, or

(ii) $m P_{h}^{0} d_{1} P_{h}^{0} d_{2} P_{h}^{0} f$ and $\left|\left\{d: d P_{h}^{0} f\right\}\right|>\kappa_{h}$.

Assume without loss of generality that (i) holds. Consider a preference profile $P$ such that

(i) $\lambda P_{f} h$,

(ii) $h P_{m} \lambda$,

(iii) $(h, h) P_{c}(\lambda, \lambda)$,

(iv) for all $h^{\prime} \in H \backslash\{h\}$ either $h P_{f} h^{\prime}$, or $\mid\left\{d: d P_{h^{\prime}}^{0} f\right.$ and $\left.r_{1}\left(P_{d}\right)=h^{\prime}\right\} \mid=\kappa_{h^{\prime}}$,

(v) for all $h^{\prime} \in H \backslash\{h\}$ either $\lambda P_{m} h^{\prime}$, or $\mid\left\{d: d P_{h^{\prime}}^{0} m\right.$ and $\left.r_{1}\left(P_{d}\right)=h^{\prime}\right\} \mid=\kappa_{h^{\prime}}$,

(vi) $r_{1}\left(P_{d_{1}}\right)=r_{1}\left(P_{d_{2}}\right)=h$, and 
(vii) $\mid\left\{d: d P_{h}^{0} m\right.$ and $\left.r_{1}\left(P_{d}\right)=h\right\} \mid=\kappa_{h}$. Note, that this also includes $d_{1}$ and $d_{2}$.

But it trivially follows from the given preference profile that for a stable matching $\mu$, for all $h^{\prime} \in H \backslash\{h\}, h^{\prime} \notin\{\mu(f), \mu(m)\}$. Thus, by our construction, a stable matching exists for this matching problem if and only if there is a stable matching for Example 3.2. However, since there does not exist a stable matching for Example 3.2, thus we do not have a stable matching for $P$. This completes the proof of part (ii) of Theorem 3.2 .

From the example above, it is not clear if non-aversion to couple diversity can always lead to a preference profile with no stable matching when doctors prefer to be matched any hospital than being unemployed. We show by the means of an example that if all the doctors are averse to unemployment, then we can not always obtain an extension of $P_{H}^{0}$ with no stable matching, when $P_{H}^{0}$ does not satisfy aversion to couple diversity.

Example 3.3. Consider a matching problem with $H=\left\{h_{1}, h_{2}\right\}$ and $D=\left\{f, m, s_{1}, s_{2}\right\}$ such that $c=\{f, m\}$ is the only couple. Let $\kappa_{h_{1}}=\kappa_{h_{2}}=2$. Thus, $\sum_{h \in H} \kappa_{h}=|D|$. The preferences of hospitals on individual doctors is given in the table below. The doctors prefer to be matched to any hospital than being unemployed.

\begin{tabular}{cc}
\hline$P_{h_{1}}$ & $P_{h_{2}}$ \\
\hline$f$ & $s_{2}$ \\
$s_{1}$ & $m$ \\
$s_{2}$ & $f$ \\
$m$ & $s_{1}$ \\
\hline
\end{tabular}

Table 3.3: Stability with Unemployment Aversion

Let $\{f, m\} P_{h_{1}}\left\{s_{1}, s_{2}\right\}$. Thus the preference $h_{1}$ does not follow aversion to couple diversity.

We show that there exists a stable matching for these preferences of hospitals for any preferences of the doctors and the couple.

Let $\mu$ be a matching for the given preferences of the hospitals such that $\mu\left(h_{1}\right)=$ $\left\{f, s_{1}\right\}$ and $\mu\left(h_{2}\right)=\left\{s_{2}, m\right\}$. Clearly, $h_{1}$ and $h_{2}$ have their top ranked doctors. Thus, neither $h_{1}$ nor $h_{2}$ would like to block $\mu$ with any other doctor. Also, no doctor would block $\mu$ with $\lambda$ as all doctors prefer being matched to any hospital than be unemployed. Thus $\mu$ is stable for any preferences of doctors even when there is no aversion for diverse couples. 
The above example leads to the following corollary.

Corollary 3.3. Suppose $h P_{d} \lambda$ for all $h \in H$ and all $d \in D$, then a stable matching always exists for any extension of $P_{H}^{0}$ when $P_{H}^{0}$ satisfies aversion to diverse couples.

\subsection{Concluding remarks}

As we have discussed earlier, existence of a stable matching is guaranteed at a preference profile if it satisfies responsiveness as defined in Klaus and Klijn (2005). The different result in this chapter stems from the fact that we allow for a setwise blocking notion, which allows for the existence of more blocking coalitions. Here, a hospital is allowed to replace two doctors by a couple, whilst Klaus and Klijn consider pairwise blocking. Thus, if a couple wishes to block with a hospital, both the members of the couple will be considered separately by the hospital instead of considering the couple as a whole. We explain this in detail in the following paragraph.

Consider Example 3.1 and Example 3.2. According to the model in Klaus and Klijn (2005), given the preference $\bar{P}_{h_{1}}$, the pair $\{f, m\}$ can not block with $h_{1}$ to remove the pair of doctors $\left\{d_{1}, d_{2}\right\}$. By their blocking notion, each member of the couple can only replace a doctor who is ranked lower to that member of the couple. The fact that $d_{1}$ and $d_{2}$ are ranked in-between $f$ and $m$, prevent the couple to block with $h_{1}$.

Now, consider the matching $\mu_{1}$ for Example 3.1 and $\mu_{2}$ for Example 3.2 such that $\mu_{1}(c)=\left(h_{2}, h_{3}\right), \mu_{1}\left(d_{1}\right)=\mu_{1}\left(d_{2}\right)=h_{1}$ and $\mu_{2}(c)=(\lambda, \lambda), \mu_{2}\left(d_{1}\right)=\mu_{2}\left(d_{2}\right)=h_{1}$. Note that, in our model, both these matchings are blocked by $\left(\left(h_{1}, h_{1}\right), c\right)$. However, this block is not possible according to the model in Klaus and Klijn (2005). It can be verified that $\mu_{1}$ and $\mu_{2}$ are indeed stable according to their model.

In this chapter, we have shown that the existence of a stable matching is not guaranteed when couples and/or hospitals have complete and responsive preferences. We have provided (a) necessary and sufficient conditions on couples' preferences so that a stable matching exists at every extension of those preferences, and (b) necessary and sufficient conditions on hospitals' preferences so that a stable matching exists at every extension of those preferences. Additionally, we have provided algorithms that produce a stable matching whenever that exists in this framework. 


\section{Part II}

\section{Social Choice Theory}



CHAPTER 4

\section{Collective Choice Rules on Convex Restricted Domains}

\subsection{Introduction}

One attempt to avoid the well-known impossibility theorems of Arrow and GibbardSatterthwaite on collective decision making is dropping the unrestricted domain condition. It means that the individuals taking part in this collective decision potentially may have any preference of a certain kind, e.g. linear orderings or weak orderings over the collective alternatives on which has to be decided. Relaxing this condition leads to so-called restricted domains. Here agents potentially may only have preferences from pre-specified subsets of the set of linear orders or it leads to domains for collective decision making in which all preferences are potentially possible but certain combinations of these are only allowed. However, restricting the domain might still lead to impossibility results, thus demonstrating that the equivalence between non-manipulability and dictatorship is far more robust than suggested by the above mentioned impossibility theorems. (See e.g. Aswal e.a.[2003] and Sato[2010]). Here like in e.g. Kalai e.a.[1977] and Kalai e.a.[1980] and Ritz[1985] we investigate do- 
mains allowing 'reasonable' collective decision rules, where at these domains the individual preferences may be chosen independently. Hence, every individual is characterised by a set of admissible preferences being a subset of all possible preferences. Moreover, we assume that any preference which is an intermediate of two admissible preferences is admissible as well.

There are many studies on domain restrictions (See for a general overview e.g. Gaertner [2002]). Examples on restrictions of combinations of individual preferences can for instance be found in the well-known literature on characterising domains which allow for consistent pairwise majority decisions. (See e.g. Sen e.a.[1969] and Inada [1964]). A well-known example on restrictions which allow for independent individual preferences is that of single peaked preferences.(See e.g. Arrow[1978], Black[1948] and Moulin[1980] being just an arbitrary choice of this strand of literature). Another example of such restrictions is that of single dipped preferences. (See e.g. Barberà e.a. [2012] where they characterise the set of all individual and group strategy-proof rules on this domain).

Having two preferences by repeatedly swapping consecutive ordered pairs of alternatives the one preference can be obtained from the other. The domain restriction imposed in this chapter demands that all the intermediate results in this swapping process are admissible if of course the starting two preferences are admissible. In this way we guarantee a natural assumption that the set of admissible preferences of an individual allows for any "continuous" change of these preferences. For instance let at one admissible preference alternative $a$ be strictly preferred to $b$ and $b$ strictly to $c$ (assuming transitivity we also have that $a$ is strictly preferred to $c$ ) and at an other preference let $c$ be strictly preferred to $a$ and $a$ strictly to $b$. So, in both preferences $a$ is strictly preferred to $b$, where in the one $c$ is worst among these three and in the other $c$ is best. As both these preferences are admissible it is natural to assume that also the intermediate of these two where $c$ is ordered between $a$ and $b$ is admissible. Precisely this is captured by the requirement that the intermediate of two admissible preferences is admissible.

Consider the Kemeny distance between preferences. For any two preferences it counts the pairs that are differently ordered in these two preferences (see also Kemeny e.a. [1962]). It is easy to see that a preference is intermediate of two given preferences if the Kemeny distance between these two equals the sum of the Kemeny distances from each of these to the intermediate. That is, the triangle inequality holds with equality. Geometrically speaking this intermediate is on the segment between the other two. Hence, the condition on the intermediate is just a convexity requirement with respect to the Kemeny distance. That is we demand that the set of admissible 
preferences is a convex subset of the set of all preferences. Clearly such a convex set may range from the set of all preferences, the least restrictive situation as there is no restriction imposed, to a singleton set the most restrictive case. It appears that a convex subset of the set of linear orderings, i.e. the set of all complete, antisymmetric and transitive relations, can be characterised as follows. For every convex set there is a partial ordering, i.e. reflexive, antisymmetric and transitive relation, which is contained in all preferences belonging to this set and moreover any linear ordering containing this partial ordering belongs to this convex set. So, a subset of the set of linear orderings is convex if, and only if, there is a partial ordering such that this convex set equals the set of all linear orderings that contain this partial ordering.

Next consider the case of restricted domains where the sets of individually admissible preferences are convex subsets of the set of linear orderings. The set of admissible preferences of a specific individual is characterised by a partial ordering that is contained in all its admissible preferences. Therefore this partial ordering can be seen as a part of the preference of that individual which is known in advance. It is called $a$ priori information. Hence, the restricted domain at hand is completely determined by these individual partial orderings. A natural question is which combination of individual partial orderings determines a restricted domain allowing for non-dictatorial, unanimous and strategy-proof choice rules. We will answer this question with respect to the union of all these partial orderings: the united a priori information. In Storcken[1985] a similar question is studied with respect to Arrow-like welfare functions. These are Pareto-optimal, non-dictatorial welfare functions which are independent of irrelevant alternatives. Roughly speaking such a restricted domain admits Arrow-like welfare functions if not all free triples with respect to the united a priori information are connected. Here a free triple with respect to this a priori information is a triple of alternatives which has no ordered pair of different alternatives in common with this united a priori information. This means that all agents may order these three alternatives in all six possible ways. In this chapter the characterisation looks at least more restrictive. Loosely speaking these domains allow for unanimous, non-dictatorial and strategy-proof choice rules if there are (at most) two undominated alternatives with respect to the united a priori information. Moreover, the choice rule now is just a monotonic choice rule between these two alternatives.

This difference in conditions on the united a priori information between the welfare function case and the choice function case stems primarily from the non-dictatorship requirement. Contrary to a choice function at a welfare function an individual might not be a dictator even if his best alternative is always ordered best in the social ranking as long as somewhere the rest of his preferences is not always copied as the social ranking. Moreover, as the domain in this setting is possibly restricted, not all al- 
ternatives might be ranked best by this individual. Lemma 4.3 however shows that this domain does not allow for unanimous, non-dictatorial and strategy-proof choice rules. This shows that although the earlier paper of Storcken[1985] and the present work are based on strongly related ideas, they seem logically independent.

Indeed Lemma 4.1 and 4.2 show that in case there are at least three undominated alternatives with respect to the united a priori information then only dictatorial choice rules are unanimous and strategy-proof. Having this result it is straight forward to see that essentially the case of two undominated alternatives with respect to the united a priori information may yield the possibility of non-dictatorial, unanimous and strategy-proof choice rules. Rules that are unanimous and monotonic choices between two alternatives. The cases of one and no undominated alternatives are reduced to the two undominated alternatives case. This however may fail as the example above on one undominated alternative $a$ demonstrates. Lemma's 4.3 and 4.4 are on necessary and sufficient conditions in order to transform these cases to the case of two undominated alternatives.

There have been many studies on domain restrictions which have tried to characterise the preference domains on which some specific non-dictatorial choice rule is strategy-proof. (See e.g. Barbie e.a.[2006] for Borda count, and Sanver[2009] for plurality rule). There have also been studies which have tried to find out necessary conditions for strategy-proofness and then have tried to find the domain restrictions to satisfy those conditions. (See e.g. Barberà e.a. [2012]). This chapter differs from these papers, as the domain restriction does not depend on a specific choice rule or certain set of conditions. We already have a restricted domain, where the nature of choice rule depends on the united a priori information of the individuals. However, the biggest difference between this chapter and other works on restricted domains, stems from the fact that different individuals can have different preference domains based on the different a priori information. This can be justified, as different individuals may have different domain of preferences for the same set of alternatives. For example, an individual of a particular religion might be averse to certain kind of meat and would always prefer any other food item over it. This leads to a priori information where the alternative of meat is always dominated by any other alternative of food item. However, this a priori information might be completely different the from priori information of another individual having some other dietary preference or restriction. Similarly, political issues can be ordered in a differently for different individuals based on their beliefs on religion, abortion, freedom of speech etc. This in turn would lead to completely different convex preference domains for the individuals. Thus, the fact that different individuals can have different convex restricted domain makes our result applicable to a wide range of examples. 
This chapter is organised as follows. Besides some basic concepts on preferences section 4.2 is on the character of convex subsets of the set of linear orderings. In section 4.3 the decision model on convex restricted domains is introduced. Section 4.4 discusses sufficient and necessary conditions such that these domains admit nondictatorial, unanimous and strategy-proof choice rules. In section 4.5 , we consider the application to a model where alternatives belong on a Euclidean plane. Section 4.6 concludes.

\subsection{Convex sets of preferences}

Let $A$ denote a non-empty and finite set of alternatives and $R$ a relation on $A$. For a non-empty subset $B$ of $A$ the restriction of $R$ to $B$ is denoted by $\left.R\right|_{B}$. The best alternatives with respect to $R$ are defined by best $(R)=\{x \in A \mid(x, y) \in R$ for all $y \in A\}$. The undominated alternatives with respect to $R$ are defined by undom $(R)=\{x \in$ $A \mid(y, x) \notin R$ for all $y \in A-\{x\}\}$. Let $\mathbb{L}(A)$ denote the set of all linear orderings on $A$, i.e. all reflexive, transitive, complete and anti-symmetric relations on $A$. Cardinality of a set $S$ is denoted by $\# S$.

Let $R^{1}, R^{2}$ and $R^{3}$ be three linear orderings on $A$. Let $B$ be a non-trivial subset of $A$. The relation $\left.\left.R^{1}\right|_{B} \gg R^{2}\right|_{A-B}=\left.\left.R^{1}\right|_{B} \cup R^{2}\right|_{A-B} \cup(B \times(A-B))$ is a relation in which the alternatives in $B$ are ordered as in $R^{1}$, the alternatives in $A-B$ are ordered as in $R^{2}$, and in which all alternatives in $B$ are strictly preferred to all alternatives in $A-B$. As a convention we write $\left.R^{1}\right|_{x}$ instead of $\left.R^{1}\right|_{\{x\}}$ for alternatives $x$. Further, instead of $(x, y) \in R^{1}$ and $(y, x) \notin R^{1}$ we also write $R^{1}=\ldots x \gg \ldots y \ldots$ The notation $R^{1}=\ldots x \gg y \ldots$ means that $(x, y) \in R^{1}$ and $(y, x) \notin R^{1}$ and in addition there are no alternatives $z$ which are ordered in between $x$ and $y$. The Kemeny distance ${ }^{1}$ between $R^{1}$ and $R^{2}$ is defined by

$$
\delta\left(R^{1}, R^{2}\right):=\frac{1}{2} \#\left[\left(R^{1}-R^{2}\right) \cup\left(R^{2}-R^{1}\right)\right] .
$$

This distance equals the number of switches needed to change relation $R^{1}$ into $R^{2}$. We will call a single setup an elementary change, i.e. $R^{1}$ and $R^{2}$ form such an elementary change if there are two different alternatives $a$ and $b$ such that $R^{1}=\left(R^{2} \cup\{(a, b)\}\right)-$ $\{(b, a)\}$. Linear ordering $R^{3}$ is said to be between $R^{1}$ and $R^{2}$ if it is on a shortest path

\footnotetext{
${ }^{1}$ We take here half the Kemeny distance because between complete anti-symmetric relations this distance is an even number.
} 
from $R^{1}$ to $R^{3}$, i.e. $\delta\left(R^{1}, R^{2}\right)=\delta\left(R^{1}, R^{3}\right)+\delta\left(R^{3}, R^{2}\right)$. In that case $R^{3}$ can be seen as an intermediate in the change of $R^{1}$ into $R^{2}$ by reversing pairs of alternatives. Clearly this equality holds if, and only if, $\left(R^{1} \cap R^{2}\right) \subseteq R^{3} \subseteq\left(R^{1} \cup R^{2}\right)$. A set of linear orderings say $V$ is said to be convex if for all $R^{1}, R^{2}$ in $V$ and all $R^{3}$ in $\mathbb{L}(A)$ if $R^{3}$ is between $R^{1}$ and $R^{2}$, then $R^{3}$ is in $V$. The following proposition characterises convexity of sets of preferences.

Proposition 4.1. Let $V$ be a set of linear orderings. Then $V$ is a convex set if, and only if, there is a partial ordering say $P$ on A, i.e. transitive, reflexive and anti-symmetric, such that

$$
V=\{R \in \mathbb{L}(A) \mid P \subseteq R\}
$$

Proof. (if-part) Let $V=\{R \in \mathbb{L}(A) \mid P \subseteq R\}$. Furthermore let $R^{1}, R^{2} \in V, R^{3}$ in $\mathbb{L}(A)$ such that $R^{3}$ is between $R^{1}$ and $R^{2}$. It is sufficient to prove that $R^{3}$ is in $V$. Because $R^{3}$ is between $R^{1}$ and $R^{2}$ we have $\left(R^{1} \cap R^{2}\right) \subseteq R^{3}$. As $R^{1}, R^{2} \in V=\{R \in \mathbb{L}(A) \mid P \subseteq R\}$ it follows that $P \subseteq\left(R^{1} \cap R^{2}\right)$. So, $P \subseteq\left(R^{1} \cap R^{2}\right) \subseteq R^{3}$. So, $R^{3} \in\{R \in \mathbb{L}(A) \mid P \subseteq R\}=V$.

(only-if-part) Let $V$ be a convex set of linear orderings. Define $P=\cap\{R \mid R \in V\}$. As all relations in $V$ are reflexive, transitive and anti-symmetric $P$ has these three properties. Now by definition of $P$ it follows that $V \subseteq\{R \in \mathbb{L}(A) \mid P \subseteq R\}$. We have to prove that $\{R \in \mathbb{L}(A) \mid P \subseteq R\} \subseteq V$. To the contrary suppose this is not the case. Then we may find an elementary change, say $R^{1}$ and $R^{2}$ such that $R^{1} \in V$ and $R^{2} \in$ $\{R \in \mathbb{L}(A) \mid P \subseteq R\}-V$. So, there are two different alternatives say $a$ and $b$ such that $R^{1}=\left(R^{2} \cup\{(a, b)\}\right)-\{(b, a)\}$. As $V \subseteq\{R \in \mathbb{L}(A) \mid P \subseteq R\}$ it follows that both $R^{1}$ and $R^{2}$ are in $\{R \in \mathbb{L}(A) \mid P \subseteq R\}$. Hence, neither $(a, b)$ nor $(b, a)$ is in $P$. Now by the definition of $P$ and by the convexity of $V$ we may assume the existence of two preferences say $R^{3}$ and $R^{4}$ in $V$ such that $R^{3}=\left(R^{4} \cup\{(a, b)\}\right)-\{(b, a)\}$, that is these two preferences form an elementary change in this same pair $a$ and $b$. Now $\left(R^{1} \cap\right.$ $\left.R^{4}\right) \subseteq R^{1}-\{(a, b),(b, a)\} \subseteq R^{2} \subseteq R^{1} \cup\{(a, b),(b, a)\} \subseteq\left(R^{1} \cup R^{4}\right)$, which means that $R^{2}$ is between $R^{1}$ and $R^{4}$. But then by the convexity of $V$ we derive the contradiction $R^{2} \in V$.

\subsection{Convex restricted domains}

Consider collective choice rules involving finite sets of alternatives $A$ and agents $N=\{1,2, \ldots n\}$ both containing at least two elements. To an agent $i$ we associate $\mathbb{V}^{i}$ a convex subset of the set of linear orderings $\mathbb{L}(A)$. This set $\mathbb{V}^{i}$ represents the set of 
admissible preferences of this agent. By Proposition 4.1 there is a partial ordering $P^{i}$ such that $\mathbb{V}^{i}=\mathbb{L}\left(P^{i}, A\right)=\left\{R \in \mathbb{L}(A) \mid P^{i} \subseteq R\right\}$. So, this partial ordering $P^{i}$ may be considered as the a priori information we have about agent $i$ 's preference. Let $\mathbb{V}^{N}$ denote the set of all profiles $p$ of individual admissible preferences. The profile $p$ assigns to every agent $i$ a preference $p(i)$ in $\mathbb{V}^{i}$. A (collective) choice rule is a function $f$ from $\mathbb{V}^{N}$ to $A$. It assigns to every profile of individual admissible preferences $p$, a collective choice $f(p)$.

Hereafter choice rules $f$ are studied with respect to the following five conditions:

Unanimity: $f(p)=a$ for all profiles $p$ and alternatives $a$ such that $\operatorname{best}(p(i))=\{a\}$ for all agents $i$,

Strategy-proofness: $(f(p), f(q)) \in p(j)$ for all agents $j$ and all $j$-deviations $p$ and $q$, i.e. $p, q \in \mathbb{V}^{N}$ with $\left.p\right|_{N-\{j\}}=\left.q\right|_{N-\{j\}}$,

Maskin Monotonicity: for all profiles $p$ and $q$ such that $(f(p), x) \in p(i)$ implies $(f(p), x) \in q(i)$ for all agents $i$ and all alternatives $x$ we have that $f(q)=f(p)$,

Strong Positive Association: for profiles $p$ and $q$ and alternatives $a$ if for all agents $i$ in $N,\left.p(i)\right|_{A-\{a\}}=\left.q(i)\right|_{A-\{a\}}$ and $(a, b) \in p(i)$ implies $(a, b) \in q(i)$ for all alternatives $b \in A$, then $f(q) \in\{a, f(p)\}$.

Non-image-dictatorship: for all agents $j$ there are profiles $p$ such that $f(p) \neq$ $\operatorname{best}\left(\left.p(j)\right|_{f\left(\mathbb{V}^{N}\right)}\right)$, where $f\left(\mathbb{V}^{N}\right)=\left\{f(p) \mid p \in \mathbb{V}^{N}\right\}$ is the range of $f$.

Strategy-proofness, Maskin monotonicity, unanimity and strong positive association are standard in literature. We will not comment on these further. Usually when we talk of non-dictatorship, it is defined as follows

for all agents $j$ there are profiles $p$ such that $f(p) \neq \operatorname{best}(p(j))$.

Omitting the restriction to the image of $f$ would yield that many convex restricted domains $\mathbb{V}^{N}$ admit odd unanimous, strategy-proof and non-dictatorial choice rules. For instance if there are agents $i$ and $j$ and alternatives $a$ and $b$ such that $(a, b) \in P^{i}$ and $b \in \operatorname{undom}\left(P^{j}\right)$. In that case define $f$ for an arbitrary profile as follows

$$
f(p)=\operatorname{best}\left(\left.p(j)\right|_{A-\{b\}}\right) .
$$

This choice rule is strategy-proof, unanimous and image-dictatorial with imagedictator $j$, but it is non-dictatorial as $b$ is not chosen if it is $j$ 's best alternative. 
Strategy-proofness follows by the dictatorial nature of this rule. Note that unanimity does not apply to $b$. So, obviously the choice rule is unanimous. Clearly nonimage-dictatorship is a stronger condition than non-dictatorship, because $f(p)=$ $\operatorname{best}(p(j))$ implies $\operatorname{best}(p(j)) \in f\left(\mathbb{V}^{N}\right)$ and therefore $f(p)=\operatorname{best}(p(j))$ implies $f(p)=\operatorname{best}\left(\left.p(j)\right|_{f\left(\mathbb{V}^{N}\right)}\right)$. To avoid odd possibilities like discussed above we strengthened non-dictatorship to non-image-dictatorship.

Remark 4.1. Because of the convexity of the domain of individual preferences and the anti-symmetry condition on these individual preferences it follows that strategyproofness, Maskin monotonicity and strong positive association are all three equivalent to each other. See also Bochet ea.[2005].

Remark 4.2. Note that for strategy-proof choice rules $f$ from $\mathbb{V}^{N}$ to A and profiles $p$ and $q$ such that $\left.p\right|_{f\left(\mathbb{V}^{N}\right)}=\left.q\right|_{f\left(\mathbb{V}^{N}\right)}$ we have that $f(p)=f(q)$.

\subsection{Possibilities under Unanimity}

In this section we investigate convex restricted domains $\mathbb{V}^{N}$ which allow for strategyproof, unanimous and non-image-dictatorial choice rules. Therefore, call $\mathbb{V}^{N}$ a possibility domain if there exists a unanimous, strategy-proof and non-image-dictatorial choice rule $f$ from $\mathbb{V}^{N}$ to $A$. Let $P^{*}=\cup\left\{P^{i} \in \mathbb{V}^{i} \mid i \in N\right\}$ the union of all a priori information. Related to $P^{*}$ we will formulate necessary and sufficient conditions for convex restricted domains such that these are possibility domains.

The following example deals with a possibility domain that essentially is a representation of the format of any possibility domain in case there are precisely two undominated alternatives.

Example 4.1. Let undom $\left(P^{*}\right)=\{a, b\}$, where $a \neq b$. Now any non-image-dictatorial monotonic choice rule on these two alternatives yields a unanimous, strategy-proof and non-image-dictatorial choice rule on $\mathbb{V}^{N}$. To be more explicit let $W_{a}$ and $W_{b}$ be two sets of subsets of $N$ such that for $x \in\{a, b\}$ and for $S \subseteq T \subseteq N$

1. none of the sets in $W_{x}$ is a singleton,

2. $N \in W_{x}$,

3. if $S \in W_{x}$ and $S \subseteq T \subseteq N$, then $T \in W_{x}$, 
4. $S \in W_{a}$ if, and only if, $(N-S) \notin W_{b}$.

Define choice rule $f$ from $\mathbb{V}^{N}$ to A for a profile $p$ as follows

$$
\begin{aligned}
f(p) & =\quad a \text { if }\{i \in N \mid(a, b) \in p(i)\} \in W_{a} \\
& =b \text { if }\{i \in N \mid(b, a) \in p(i)\} \in W_{b} .
\end{aligned}
$$

Now $f$ is well-defined because of condition (4). Strategy-proofness follows because of the monotonicity condition (3) and non-image-dictatorship because of condition (1). As $a$ and $b$ are the only undominated elements of $P^{*}$ unanimity has no bite on the other alternatives. Unanimity on a and $b$ now follows from condition (2).

Example 4.1 shows that if the united a priori information $P^{*}$ has two undominated elements, then the domain allows for strategy-proof, non-image-dictatorial and unanimous choice rules. Let $U=\operatorname{undom}\left(P^{*}\right)$. It appears that the cardinality $\# U$ of $U$ plays an important role in whether or not a domain at hand allows for non-image-dictatorial, unanimous and strategy-proof choice rules. For agents $i$ let $\widetilde{P}^{i}=P^{i} \cup(U \times(A-U))$ and $\widetilde{\mathbb{V}}^{i}=\mathbb{L}\left(\widetilde{P}^{i}, A\right)$. Because $P^{i} \subseteq \widetilde{P}^{i}$, it follows that $\widetilde{\mathbb{V}}^{i} \subseteq \mathbb{V}^{i}$ for all agents $i$. Note that at profiles in $\widetilde{\mathbb{V}}^{N}$ all agents prefer all alternatives in $U$ to all alternatives which are not in $U$. As the alternatives in $U$ are undominated elements of the union of all a priori information $P^{*}$ it follows that no agent a priori prefers any among these. So, if we would restrict all linear orderings in $\widetilde{\mathbb{V}}^{i}$ to $U$ then we would obtain the set of all linear orderings on $U:\left.\widetilde{\mathbb{V}}^{i}\right|_{U}=\mathbb{L}(U)$.

For the four cases $\# U \geq 3, \# U=2, \# U=1$ and $\# U=0$ we will investigate the possibilities of non-image-dictatorial, unanimous and strategy-proof choice rules from $\mathbb{V}^{N}$ to $A$. Let $h$ be a unanimous and strategy-proof choice rule from $\mathbb{V}^{N}$ to $A$. For the case $\# U \geq 3$ the following two lemmas yield that $h$ is image-dictatorial.

Lemma 4.1. Let $\# U \geqslant 3$. Then there is an agent, say $j$, such that for all profiles $p$ in $\mathbb{V}^{N}$ and all alternatives $x \in U$ if best $(p(j))=x$, then $h(p)=x$.

Proof. Let $\widetilde{h}=\left.h\right|_{\widetilde{\mathbb{V}}^{N}}$. As $\widetilde{h}$ inherits unanimity and strategy-proofness from $h$, it follows that $\widetilde{h}$ is Maskin monotone and unanimous. From this we may easily deduce that $\widetilde{h}\left(\widetilde{\mathbb{V}}^{N}\right)=U$. Moreover, note that by Maskin monotonicity it now follows that for profiles $p$ and $q$ in $\widetilde{\mathbb{V}}^{N} \widetilde{h}(p)=\widetilde{h}(q)$ if $\left.p\right|_{U}=\left.q\right|_{U}$. So, $\widetilde{h}$ can be seen as a choice rule on $\left.\widetilde{\mathbb{V}}^{N}\right|_{U}=\mathbb{L}(U)^{N}$. Gibbard-Satterthwaite [1973,1975] yields that $\widetilde{h}$ is dictatorial, with dictator say $j$. Now let $p$ be a profile in $\mathbb{V}^{N}$ with $\operatorname{best}(p(j))=x \in U$. 
It is sufficient to prove $h(p)=x$. Take $y \in U-\{x\}$ and profiles $q$ and $r$ in $\mathbb{V}^{N}$ such that $q(j)=\left.x \gg y \gg p(j)\right|_{A-\{x, y\}}, r(j)=\left.\left.x \gg y \gg p(j)\right|_{U-\{x, y\}} \gg p(j)\right|_{A-U}$, $q(i)=\left.y \gg p(i)\right|_{A-\{y\}}$ and $r(i)=\left.\left.y \gg p(i)\right|_{U-\{y\}} \gg p(i)\right|_{A-U}$ for agents $i$ different from $j$. As $r \in \widetilde{\mathbb{V}}^{N}$ and $\widetilde{h}$ is dictatorial with dictator $j$ it follows that $h(r)=x$. Strategyproofness and unanimity imply that $h(q) \notin A-\{x, y\}$. So, $h(q) \in\{x, y\}$. As $h(r)=x$ Maskin monotonicity implies that $h(q) \neq y$. So, $h(q)=x$. By Maskin monotonicity this implies $h(p)=x$.

Lemma 4.1 shows that if there are enough undominated elements with respect to the union of a priori information, then there is an agent which is image-dictatorial on all these undominated elements. The lemma hereafter shows that this decisiveness spreads on all alternatives in the image of choice rule $h$.

Lemma 4.2. Let $\# U \geqslant 3$. Then there is an agent, say $j$, such that for all profiles $p$ in $\mathbb{V}^{N}$ and all alternatives $x \in h\left(\mathbb{V}^{N}\right)$ if best $\left(\left.p(j)\right|_{h\left(\mathbb{V}^{N}\right)}\right)=x$, then $h(p)=x$. Hence, $h$ is image-dictatorial with image-dictator $j$.

Proof. Let $j$ be as in Lemma 4.1. Let $x \in h\left(\mathbb{V}^{N}\right)$ and best $\left(\left.p(j)\right|_{h\left(\mathbb{V}^{N}\right)}\right)=x$. As $x \in$ $h\left(\mathbb{V}^{N}\right)$ there is a profile $w \in \mathbb{V}^{N}$ with $h(w)=x$. We will construct a list of profiles starting with $w$ and ending at $p$ and prove for all these profiles that their image is $x$ under $h$. Consider profile $v$ a $j$-deviation of $w$ such that $v(j)=p(j)$ and $v(i)=$ $w(i)$ for $i \neq j$. Because of strategy-proofness and $\operatorname{best}\left(\left.p(j)\right|_{h\left(\mathbb{V}^{N}\right)}\right)=x$ it follows from $h(w)=x$ that $h(v)=x$. Now let $a$ and $b$ be two different alternatives in $U$. Without loss of generality assume that $(b, a) \in p(j)$. Consider profile $u$ defined for all agents $i$ different from $j$ by $u(i)=\left.b \gg v(i)\right|_{A-\{b\}}$. Let $u(j)$ be obtained from $v(j)$ by shifting $a$ just below $x$ and $b$ just below $a: u(j)=\ldots x \gg a \gg b \ldots$ So, $\left.u(j)\right|_{A-\{a, b\}}=$ $\left.v(j)\right|_{A-\{a, b\}}$ and $u(j)$ ranks $x$ just above $a$ just above $b$. Let $\tilde{u}$ be the $j$-deviation of $v$ such that $\widetilde{u}(j)=u(j)$. Because of $h(v)=x$, Maskin monotonicity implies that $h(\widetilde{u})=x$. But then $h(u) \neq a$, because otherwise Maskin monotonicity would imply that $h(\widetilde{u})=a$ if $h(u)=a$. To see that $h(u) \neq b$ consider profile $\widehat{u}$ a $j$-deviation of $u$ such that $\widehat{u}(j)=\left.a \gg u(j)\right|_{A-\{a\}}$. By Lemma 4.1 we have that $h(\widehat{u})=a$. Now $h(u) \neq$ $b$, because otherwise Maskin monotonicity would imply that $h(\widehat{u})=b$ if $h(u)=b$. Finally unanimity and strategy-proofness imply that $h(u) \notin h\left(\mathbb{V}^{N}\right)-\{a, b, x\}$. So, $h(u)=x$. Let profile $r$ be the $j$-deviation of $u$ such that $\left.r(j)\right|_{A-\{b\}}=\left.p(j)\right|_{A-\{b\}}$ and $\left.r(j)\right|_{A-\{a\}}=\left.u(j)\right|_{A-\{a\}}$. That is $r(j)$ is obtained from $p(j)$ by shifting $b$ just below $x$. Maskin monotonicity implies that $h(r)=x$. Let $q$ be the profile such that $q(j)=r(j)$ and for all agents $i$ different from $j$ let $q(i)=\left.b \gg p(i)\right|_{A-\{b\}}$. Maskin monotonicity and $h(r)=x$ implies $h(q) \neq b$. Strategy-proofness and unanimity imply that $h(q) \notin$ $h\left(\mathbb{V}^{N}\right)-\{b, x\}$. So, $h(q)=x$ and Maskin monotonicity now implies $h(p)=x$. 
For the case $\# U=2$ Example 4.1 shows that there are non-image-dictororial, unanimous and strategy-proof choice rules. Example 4.2 below shows a case in which such choice rules exist in case $\# U=1$. Moreover, the sufficiency condition implicitly spelled out by this example appears to be necessary as is shown in Lemma 4.3 below.

Example 4.2. Let $U=\{a\}$ and let $b \in A-\{a\}$ such that $(a, b) \notin P^{1} \cup P^{2}$. Define choice rule $f$ from $\mathbb{V}^{N}$ to A for a profile $p$ as follows

$$
\begin{aligned}
f(p) & =b \text { if both }(b, a) \in p(1) \text { and }(b, a) \in p(2) \\
& =a \text { in all other cases. }
\end{aligned}
$$

Clearly $f$ is non-image-dictatorial and because $U=\{a\}$ unanimity has no bite on alternatives different from a. Hence, by definition it follows that $f$ is unanimous. Strategy-proofness follows because $f$ is essentially a monotonic choice rule between two alternatives.

Actually in Example 4.2, we defined a choice rule on two alternatives and two agents where neither the rest of the agents nor the rest of the alternatives can influence the outcome. These act just like dummies. It is clear however that we may define choice rules at which more agents, say $j$, can have an impact on the outcome if for such agents $(a, b) \notin P^{j}$. It is also clear that if there is only one agent say agent 1 for which $(a, b) \notin P^{1}$ the choice rule defined in Example 4.2 is image-dictatorial. The following Lemma 4.3 shows that if $U=\{a\}$ for some alternative $a$, then the existence of an alternative $b \neq a$ such that for two agents $i$ and $j(a, b) \notin P^{i} \cup P^{j}$ is not only a sufficient but also a necessary condition such that $h$ is non-image-dictatorial.

Lemma 4.3. Let $U=\{a\}$ for some alternative $a$ in $A$. Then the following two are equivalent

1. There are different agents $i$ and $j$ and an alternative $b \in A-\{a\}$ such that $(a, b) \notin P^{i} \cup P^{j}$;

2. There exist unanimous, strategy-proof and non-image-dictatorial choice rules f from $\mathbb{V}^{N}$ to $A$.

Proof. ((1) implies (2)) This implication follows by Example 4.2.

((2) implies (1)) To prove the contra position of this implication suppose

$(\alpha)$ For all alternatives $b \in A-\{a\}$ there are agents $i_{b}$ such that $(a, b) \in P^{j}$ for all $j \in N-\left\{i_{b}\right\}$ 
( $\beta$ ) Choice rules $f$ from $\mathbb{V}^{N}$ to $A$ is unanimous and strategy-proof.

It is sufficient to prove that $f$ is image-dictatorial. By unanimity and $U=\{a\}$ it follows that $a \in f\left(\mathbb{V}^{N}\right)$. If $f\left(\mathbb{V}^{N}\right)=\{a\}$, then $f$ is constant and every agent is imagedictator of $f$. Let $\{a, b\}=f\left(\mathbb{V}^{N}\right)$ for some $b \in A-\{a\}$. In view of Remark 4.2, assumption $(\alpha)$ and unanimity of $f$ yield that for a profile $r$

$$
\begin{aligned}
f(r) & =b \text { if }(b, a) \in p\left(i_{b}\right) \\
& =a \text { in all other cases. }
\end{aligned}
$$

So, agent $i_{b}$ is image-dictator of $f$. Finally, consider the case that $f\left(\mathbb{V}^{N}\right)$ has at least three elements. Let $b, c \in f\left(\mathbb{V}^{N}\right)$ and $a, b$ and $c$ be all different. We first prove that $i_{b}=i_{c}$. Then we show that this agent is image-dictator. To the contrary assume that $i_{b} \neq i_{c}$. In view of assumption $(\alpha)$ and Maskin monotonicity for all $x \in f\left(\mathbb{V}^{N}\right)-\{a\}$ there are profiles $p_{x}$ such that $f\left(p_{x}\right)=x$, for all $j \in N-\left\{i_{x}\right\} \operatorname{best}\left(p_{x}(j)\right)=a$ and $p_{x}\left(i_{x}\right)=\ldots x \gg a \ldots$ Consider profile $q$ defined by $q\left(i_{b}\right)=p_{b}\left(i_{b}\right), q\left(i_{c}\right)=p_{c}\left(i_{c}\right)$ and best $(q(j))=a$ for all $j \in N-\left\{i_{b}, i_{c}\right\}$. By assumption $(\alpha)$ it follows that $(a, b) \in p_{c}\left(i_{c}\right)$ and $(a, c) \in p_{b}\left(i_{b}\right)$. Because $f\left(p_{b}\right)=b$, Maskin monotonicity implies $f(q) \neq a$. Let $r$ be a profile defined by $\left.q\right|_{N-\left\{i_{b}, i_{c}\right\}}=\left.r\right|_{N-\left\{i_{b}, i_{c}\right\}}, r\left(i_{b}\right)=\left.a \gg p_{b}\left(i_{b}\right)\right|_{A-\{a\}}$ and $r\left(i_{c}\right)=\left.a \gg p_{c}\left(i_{c}\right)\right|_{A-\{a\}}$. By unanimity $f(r)=a$, so Maskin monotonicity implies $(f(q), a) \in q\left(i_{b}\right)$ or $(f(q), a) \in q\left(i_{c}\right)$. Because of $f(q) \neq a$, assumption $(\alpha)$ implies either $(a, f(q)) \in q\left(i_{b}\right)$ or $(a, f(q)) \in q\left(i_{c}\right)$. Without loss of generality assume $(f(q), a) \in q\left(i_{b}\right)$ and therewith consequently $(a, f(q)) \in q\left(i_{c}\right)$. Consider profile $v$ defined by $\left.v\right|_{N-\left\{i_{b}\right\}}=\left.q\right|_{N-\left\{i_{b}\right\}}$ and $v\left(i_{b}\right)=\left.a \gg p_{b}\left(i_{b}\right)\right|_{A-\{a\}}$. Comparing $q$ and $v$, Strong Positive Association yields that $f(v) \in\{a, f(q)\}$. If $f(v)=f(q)$, then $f$ is manipulable by agent $i_{c}$ at profile $v$ towards profile $r$. If $f(v)=a$, then Maskin monotonicity implies the contradiction $f\left(p_{c}\right)=a$. Hence, $i_{b}=i_{c}$. Since $b$ and $c$ were arbitrary choices, we may conclude that there is an agent $i$ such that $i=i_{x}$ for all $x \in f\left(\mathbb{V}^{N}\right)-\{a\}$. In order to prove that $i$ is image-dictator of $f$ let $w$ be a profile in $\mathbb{V}^{N}$ such that $x=\operatorname{best}\left(\left.w(i)\right|_{f\left(\mathbb{V}^{N}\right)}\right)$. It is sufficient to prove that $f(w)=x$. To the contrary let $f(w)=y \neq x$. By assumption $(\alpha)$ we have that $(a, z) \in w(j)$ for all $z \in f\left(\mathbb{V}^{N}\right)-\{a\}$ and all $j \in N-\{i\}$. According to Remark 4.2 we may assume that $a=\operatorname{best}(w(j))$ for all $j \in N-\{i\}$. Because $f(w)=y$ it follows by unanimity and strategy-proofness that $(y, a) \in w(i)$. Consider $i$-deviation $u$ of $w$ such that $u(j)=w(j)$ for $j \in N-\{i\}, u(i)=\ldots x \gg a \ldots$ and $\left.u(i)\right|_{A-\{a\}}=\left.w(i)\right|_{A-\{a\}}$. Strong Positive Association and the assumption $x=\operatorname{best}\left(\left.w(i)\right|_{f\left(\mathbb{V}^{N}\right)}\right)$ and therewith $x=\operatorname{best}\left(\left.u(i)\right|_{f\left(\mathbb{V}^{N}\right)}\right)$ imply that $f(u) \in\{a, y\}$. As $(a, y) \in u(i)$, unanimity and strategyproofness imply that $f(u) \neq y$ in case $a \neq y$. So, $f(u)=a$. But then Maskin monotonicity implies $f\left(\bar{p}_{x}\right)=a$, where $\bar{p}_{x}(i)=u(i)$ and $\left.\bar{p}_{x}\right|_{N-\{i\}}=\left.p_{x}\right|_{N-\{i\}}$. Because $f\left(p_{x}\right)=x$ and $(x, a) \in p_{x}(i)$, where $x \neq a$, it follows that $f$ is manipulable at $\bar{p}_{x}$ by 
agent $i$ towards profile $p_{x}$. This contradiction yields that $f(w)=x$ and $f$ is imagedictatorial with image-dictator $i$.

Finally, we consider the case where $U=\emptyset$. First we introduce an example which expresses a sufficient condition. Then like in the case that $U$ is a singleton, we show that this sufficient condition is also necessary.

Example 4.3. Let $U=\emptyset$. Let $a, b \in A$, with $a \neq b$, such that $(a, b) \notin P^{1} \cup P^{2}$ and $(b, a) \notin P^{1} \cup P^{2}$. Define choice rule $f$ for a profile $p$ as follows

$$
\begin{aligned}
f(p) & =b \text { if both }(b, a) \in p(1) \text { and }(b, a) \in p(2) \\
& =a \text { in all other cases. }
\end{aligned}
$$

Like in Example 4.2 it follows that $f$ is unanimous, strategy-proof and non-imagedictatorial.

Lemma 4.4. Let $U=\emptyset$. Then the following two are equivalent

1. There are different agents $i$ and $j$ and alternatives $a, b \in A$ such that $(a, b) \notin$ $P^{i} \cup P^{j}$ and $(b, a) \notin P^{i} \cup P^{j}$;

2. There exist unanimous, strategy-proof and non-image-dictatorial choice rules f from $\mathbb{V}^{N}$ to $A$.

Proof. ((1) implies (2)) This implication follows by Example 4.3.

((2) implies (1)) To prove the contra position of this implication suppose

( $\alpha$ ) For all alternatives $a, b \in A$, with $a \neq b$ there are agents $i_{a b}$ such that for all $j \in N-\left\{i_{a b}\right\},(a, b) \in P^{j}$ or $(b, a) \in P^{j}$;

( $\beta$ ) Choice rules $f$ from $\mathbb{V}^{N}$ to $A$ is unanimous and strategy-proof.

It is sufficient to prove that $f$ is image-dictatorial. If $f\left(\mathbb{V}^{N}\right)$ is a singleton, then $f$ is a constant choice rule and all agents are image-dictator. So, let $f\left(\mathbb{V}^{N}\right)$ consist of more than one element. Because $\mathbb{V}^{N}$ is a convex restricted domain we may assume that there is an agent $i$, alternatives $a$ and $b$, with $a \neq b$, and profiles $p_{a}$ and $p_{b}$ such that $f\left(p_{a}\right)=a, f\left(p_{b}\right)=b,\left.p_{a}\right|_{N-\{i\}}=\left.p_{b}\right|_{N-\{i\}}$ and $p_{a}(i)=\left(p_{b}(i)-\{(b, a)\}\right) \cup\{(a, b)\}$. From assumption $(\alpha)$ we infer that $i=i_{a b}$. Next let $X_{a}=\left\{p \in \mathbb{V}^{N} \mid p(i)=p_{a}(i)\right\}$ 
and let $X_{b}=\left\{p \in \mathbb{V}^{N} \mid p(i)=p_{b}(i)\right\}$. We show that $f\left(X_{a}\right)=\{a\}$ and $f\left(X_{b}\right)=\{b\}$. Let $q_{a}$ and $q_{b}$ by $j$-deviations of $p_{a}$ and $p_{b}$ respectively for some agent $j \in N-\{i\}$, such that $\left.p_{a}\right|_{N-\{j\}}=\left.q_{a}\right|_{N-\{j\}},\left.p_{b}\right|_{N-\{j\}}=\left.q_{b}\right|_{N-\{j\}}$ and $q_{a}(j)=q_{b}(j)=\left(p_{a}(j)-\right.$ $\{(x, y)\}) \cup\{(y, x)\}$ for some alternatives $x, y \in A$. Because $\mathbb{V}^{N}$ is convex restricted domain, thus in order to prove $f\left(X_{a}\right)=\{a\}$ and $f\left(X_{b}\right)=\{b\}$ it is sufficient to prove that $f\left(q_{a}\right)=a$ and $f\left(q_{b}\right)=b$. Assumption $(\alpha)$ yields that $j=i_{x y}$. But as $j \neq i$ and $i=i_{a b}$, this implies by assumption $(\alpha)$ that $\{a, b\} \neq\{x, y\}$. If $a \notin\{x, y\}$, then by Maskin monotonicity we have $f\left(q_{a}\right)=a$ and $f\left(q_{b}\right) \neq a$. But as Strong Positive Association and $f\left(q_{a}\right)=a$ imply $f\left(q_{b}\right) \in\{a, b\}$, it follows that $f\left(q_{b}\right)=b$. Hence, if $a \notin\{x, y\}$, then $f\left(q_{a}\right)=a$ and $f\left(q_{b}\right)=b$. Similarly it follows that if $b \notin\{x, y\}$, then $f\left(q_{a}\right)=a$ and $f\left(q_{b}\right)=b$. Because $\{a, b\} \neq\{x, y\}$, at least one of these implications hold and therewith $f\left(q_{a}\right)=a$ and $f\left(q_{b}\right)=b$. Hence, $f\left(X_{a}\right)=\{a\}$ and $f\left(X_{b}\right)=\{b\}$.

For $R \in \mathbb{V}^{i}$ let $X_{R}=\left\{p \in \mathbb{V}^{N} \mid p(i)=R\right\}$. Hence, $X_{a}=X_{p_{a}(i)}$ and $X_{b}=X_{p_{b}(i)}$. The following two claims complete the proof.

Claim 4.1. Let $R \in \mathbb{V}^{i}$ be such that $f\left(X_{R}\right)=\{x\}$. Then $x=\operatorname{best}\left(\left.R\right|_{f\left(\mathbb{V}^{N}\right)}\right)$.

To the contrary let $x \neq y=\operatorname{best}\left(\left.R\right|_{f\left(\mathbb{V}^{N}\right)}\right)$. Then there are $p_{y} \in \mathbb{V}^{N}$ such that $f\left(p_{y}\right)=y$. Consider $i$-deviation $p_{x y}$ of $p_{y}$ such that $\left.p_{x y}\right|_{N-\{i\}}=\left.p_{y}\right|_{N-\{i\}}$ and $p_{x y}(i)=R$. Because $f\left(X_{R}\right)=\{x\}$ we have $f\left(p_{x y}\right)=x$ and herewith $f$ is manipulable by agent $i$ at $p_{x y}$ towards profile $p_{y}$.

Claim 4.2. Let $R^{1}$ and $R^{2}$ be two relations in $\mathbb{V}^{i}$ that form an elementary change in $x, y \in A$, i.e. $R^{1}=\left(R^{2}-\{(y, x)\}\right) \cup\{(x, y)\}$. Let $f\left(X_{R^{1}}\right)=\{c\}$ for some alternative $c$ in $A$. Then $f\left(X_{R^{2}}\right)=\{d\}$ for some alternative $d$ in $A$.

In case $x \neq c$ Maskin monotonicity and $f\left(X_{R^{1}}\right)=\{c\}$ imply $f\left(X_{R^{2}}\right)=\{c\}$. So, suppose $c=x$. We are also done if $f\left(X_{R^{2}}\right)=\{c\}$. As $\mathbb{V}^{i}$ is a convex restricted domain we therefore may assume that there are $p_{c} \in X_{R^{1}}$ and $p_{d} \in X_{R^{2}}$ where $c=f\left(p_{c}\right) \neq$ $f\left(p_{d}\right)=d$ and $\left.p_{c}\right|_{N-\{i\}}=\left.p_{d}\right|_{N-\{i\}}$. Now similarly to the proof of $f\left(X_{a}\right)=a$ and $f\left(X_{b}\right)=b$ it follows that $f\left(X_{R^{2}}\right)=\{d\}$.

Claims $4.1,4.2$ and the fact that $\mathbb{V}^{N}$ is a convex restricted domain imply that $f$ is image-dictatotrial.

Summarising the above results yields

Corollary 4.1. For all agents $i \mathbb{V}^{i}=\left\{R \in \mathbb{L}(A) \mid P^{i} \subseteq R\right\}$ for some partial ordering $P^{i}$ referred to as i's a priori information. Let $P^{*}=\cup\left\{P^{i} \mid i \in N\right\}$ denote the collective 
a priori information. Let $U=$ undom $\left(P^{*}\right)$ be the set of undominated elements with respect to this collective a priori information $P^{*}$. Then (1) and (2) are equivalent, where

1. There exist non-image-dictatorial, unanimous and strategy-proof choice rules f from $\mathbb{V}^{N}$ to $A$;

2. One of the following three is true:

a There are two different alternatives $a$ and $b$ such that $U=\{a, b\}$;

b There is one alternative a such that $U=\{a\}$; there is an alternative $b \in$ $A-\{a\}$ and there are two different agents, say $i$ and $j$, such that $(a, b) \notin$ $P^{i} \cup P^{j}$;

c Set $U$ is empty; there are two different alternatives, say a and $b$, and two different agents, say $i$ and $j$, such that $(a, b) \notin P^{i} \cup P^{j}$ and $(b, a) \notin P^{i} \cup P^{j}$.

\subsection{An application}

In this section we apply the main result of the previous section to a choice problem where the set of alternatives consists of ordered pair of commodity bundles, which are denoted by unique points on the Euclidean plane. This application can trivially be extended to cases where the set of alternatives consists of n-tuples of commodity bundles. Consider a set of agents $N=\{1, \ldots, N\}$ and a finite set alternatives on Euclidean plane denoted by $A$. As mentioned above, $R$ denotes a relation on $A$, and the set of linear orderings are denoted by $\mathbb{L}(A)$.

Let for any agent $i$, the set of admissible preference $\mathbb{V}^{i}$ satisfy monotonicity i.e., 'more-is-better' for all the agents. Moreover let $\mathbb{V}^{i}$ be a subset of $\mathbb{L}(A)$. For any agent $i$, more-is-better gives a priori information $P^{i}$ for the agent's preference. However this a priori information does not give a relation between all the alternative. For example, consider $a=\left(a_{1}, a_{2}\right)$ and $b=\left(b_{1}, b_{2}\right)$ such that $a_{1} \geq b_{1}$ and $a_{2}<b_{2}$, then $(a, b),(b, a) \notin P^{i}$. Thus, $\mathbb{V}^{i}=\mathbb{L}\left(P^{i}, A\right)=\left\{R \in \mathbb{L}(A): P^{i} \subseteq R\right\}$, which implies that $\mathbb{V}^{i}$ is a convex subset of $\mathbb{L}(A)$ and $\mathbb{V}^{N}$ is a convex restricted domain.

Since $\mathbb{V}^{i}$ need not be the same for each agent $i$, we can also extend the above model to the case when preferences of all the agents need not satisfy monotonicity. Thus there might be some agents, who consider the alternatives as bad, while some who consider 
them as goods. Moreover there maybe some agents who consider the alternatives as good, but their preferences might still not satisfy monotonicity, e.g. satiated preference. This gives a priori information $P^{i}$ for every agent $i$. However, $\mathbb{V}^{i}$ is a subset of $\mathbb{L}(A)$ and $\mathbb{V}^{i}=\mathbb{L}\left(P^{i}, A\right)=\left\{R \in \mathbb{L}(A): P^{i} \subseteq R\right\}$ implies that $\mathbb{V}^{i}$ is convex and $\mathbb{V}^{N}$ is a convex restricted domain.

Let $P^{*}=\cup\left\{P^{i} \mid i \in N\right\}$ and $U=\operatorname{undom}\left(P^{*}\right)$. By Corollary 4.1, there exist non-imagedictatorial, unanimous and strategy-proof choice rule $f$ from $\mathbb{V}^{N}$ to $A$ if and only if either one of the following three holds:

a There are two different alternatives $a$ and $b$ such that $U=\{a, b\}$;

b There is one alternative $a$ such that $U=\{a\}$; there is an alternative $b \in A-\{a\}$ and there are two different agents, say $i$ and $j$, such that $(a, b) \notin P^{i} \cup P^{j}$;

c Set $U$ is empty; there are two different alternatives, say $a$ and $b$, and two different agents, say $i$ and $j$, such that $(a, b) \notin P^{i} \cup P^{j}$ and $(b, a) \notin P^{i} \cup P^{j}$.

\subsection{Conclusions}

Corollary 4.1 describes the convex restricted domains which allow for unanimous, non-image-dictatorial and strategy-proof choice rules. The main requirement that there are at most two undominated alternatives with respect to the united a priori information is considerably restrictive. Therefore the characterisation can also be seen as an impossibility result. On the other hand these conditions would only change marginally if anonymity were taken instead of non-image-dictatorship. Where anonymity is a condition that imposes that the choice rule can be defined on the frequency distributions of the preferences in a profile. So, agents are treated equally. Indeed considering Example 4.1 it is easy to define anonymous choice rules on two alternatives. The case of two undominated alternative would not change at all. In case of one or no undominated alternative, however, differences with Corollary 4.1 occur, because for instance a constant rule is anonymous but also image-dictatorial.

Another condition frequently imposed on choice rules is Pareto-optimality. It means that an alternative $x$ is not chosen when there are alternatives $y$ which all agents (strictly) prefer to $x$. It is not difficult to see that the choice rules of Example 4.1 are not necessarily Pareto-optimal. There are however special cases of this example which allow for Pareto-optimal, non-image dictatorial and strategy-proof choice 
rules. To illustrate this take the same notations and assumptions as in Example 4.1. Furthermore, assume that for all $c \in A-\{a, b\}(a, c) \in P^{*}$. Now the following imputation rule $f$ is well-defined and satisfies these three conditions.

$$
\begin{aligned}
f(p) & =b \text { if }(b, a) \in p(i) \text { for all agents } i \in N \\
& =a \text { in all other cases. }
\end{aligned}
$$

Pareto-optimality is a stronger condition than unanimity. In view of the restrictive result spelled out by Corollary 4.1 we therefore did not incorporate an extensive study on convex restricted domains which allow for Pareto-optimal, non-(image)-dictatorial and strategy-proof choice rules.

Based on Corollary 4.1 and the remarks made above one might argue that changing the conditions of admissible choice rules will not lead to more general convex restricted domains which allow for such rules. Hence the concept of convexity is too restrictive after all. Indeed if we weaken the convexity condition, i.e. that all intermediate preferences of two admissible preferences are admissible, into connectedness, i.e. there is a path of intermediate preferences leading from one to the other admissible preference, then more general restricted domains may allow for "nice" choice rules. For example the domains discussed here as well those of single peaked preferences fall under this connectedness condition and both allow for acceptable choice rules. 

Bibliography

[1] Aldershof B. and Carducci O., Stable matchings with couples, Discrete Applied Mathematics 68 (1996), 203-207.

[2] Alkan A. and Gale D., Stable schedule matching under revealed preferences, Journal of Economic Theory 112 (2003), 289-306.

[3] Arrow K.J., Social choice and individual values, Yale University Press (1978), $19^{\text {th }}$ edition.

[4] Aswal N., Chatterji S. and Sen A., Dictatorial domains, Economic Theory 22 (2003), 45-62.

[5] Barberà S., Berga D. and Moreno B., Domains, ranges and strategy-proofness: the case of single-dipped preferences, Social Choice and Welfare 39 (2012), 335352.

[6] Barberà S., Berga D. and Moreno B., Two necessary conditions for strategyproofness: On what domains are they also sufficient?, Games and Economic Behaviour 75 (2012), 490-509.

[7] Barbie M., Puppe C.and Tsanádi A., Non-manipulable domains for the Borda count, Economic Theory 27 (2006), 411-430 
[8] Black D., On the rationale of group decision making, Journal of Political Economy 56 (1948), 23-34

[9] Bochet O. and Storcken T., Maximal domains for strategy-proof or Maskin monotone choice rules, METEOR research memorandum, Maastricht (2005).

[10] Cantala D., Matching Markets: the Particular Case of Couples, Economics Bulletin (2004), Vol. 3, No. 45, 1-11.

[11] Dutta B. and Massó J., Stability of matchings when individuals have preferences over colleagues, Journal of Economic Theory 75 (1997), no. 2, 464-475.

[12] Echenique F. and Oviedo J., A theory of stability in many-to-many matching markets, Theoretical Economics 1 (2006), no. 2, 233-273.

[13] Gaertner W. Restricted domains, in: Arrow K.J., Sen A.K. and Suzumura K. (ed.), Handbook of Social Choice 1, Chapter 3 (2002), 131-170, Elsevier.

[14] Gale D. and Shapley L., College Admissions and the Stability of Marriage, The American Mathematical Monthly 69 (1962), no. 1, 9-15.

[15] Gibbard A., Manipulation of voting schemes: A general result, Econometrica 41 (1973), 587-601.

[16] Gusfield D. and Irving R.W., The Stable Marriage Problem: Structure and Algorithms, MIT Press, Cambridge, MA (1989).

[17] Hatifield J. and Kojima F., Substitutes and stability for matching with contracts, Journal of Economic Theory 145 (2010), 1704-1723.

[18] Hatfield J. and Milgrom P., Auctions, matching and the law of aggregate demand, Working paper 04-003, Department of Economics, Stanford University, February 2004.

[19] Inada K, A note on the simple majority decision rule, Econometrica 32 (1964), 525-531.

[20] Kalai E. and Muller E., "Characterisation of domains admitting non-dictatorial social welfare functions and non-manipulable voting procedures", Journal of Economic Theory 16 (1977), 457-469.

[21] Kalai E. and Ritz Z., "Characterisation of the private alternatives domains admitting Arrow social welfare functions", Journal of Economic Theory 22 (1980), 23-36. 
[22] Kelso A. Jr. and Crawford V., Job matching, coalition formation, and gross substitutes, Econometrica 50 (1982), 1483-1504.

[23] Kemeny J.G. and Snell J.L., Mathematical models in social sciences, New York, Ginn and Company, (1962).

[24] Klaus B. and Klijn F., Stable matchings and preferences of couples, Journal of Economic Theory 121 (2005), no. 1, 75-106.

[25] Knuth D., Marriages Stables, Les Presses de l'Université de Montréal (1976).

[26] Kojima F., Pathak P. A., and Roth A.E., Matching with couples: Stability and incentives in large markets, Working Paper, Stanford University, 2010.

[27] Konishi H. and Ünver M.U., Credible group stability in many-to-many matching problems, Journal of Economic Theory 129 (2006), no. 1, 57-80.

[28] Martínez R., Massó J., Neme A. and Oviedo J., Single Agents and the Set of Many-to-One Stable Matchings, Journal of Economic Theory 91 (2000), 91-105.

[29] Moulin H., On strategy-proofness and single-peakedness, Public Choice, Public Choice (1980), 437-455

[30] Ritz Z., Restricted domains, Arrow social welfare functions and noncorruptable and non-manipulable social choice correspondences: The case of private alternatives, Mathematical Social Science (1985), no. 4, 155-179

[31] Roth A.E., The evolution of the labor market for medical interns and residents: A case study in game theory, The Journal of Political Economy (1984), 991-1016.

[32] Roth A.E., The college admissions problem is not equivalent to the marriage problem, Journal of Economic Theory 36 (1985), 277-288.

[33] Roth A.E. and Sotomayor M.A.O., Two Sided Matching: A Study in GameTheoretic Modelling and Analysis, Economic Monograph Series, Cambridge Univ. Press, Cambridge (1990).

[34] Sanver M.R., Strategy-proofness of the plurality rule over restricted domains, Economic Theory 39 (2009), 461-471.

[35] Sato S., Circular domains, Review of Economic Design 14 (2010), 331-342.

[36] Satterthwaite M.A., Strategy-proofness and Arrow's conditions: Existence and correspondence theorem for voting procedures and social welfare functions, Journal of Economic Theory 10 (1975), 187-217. 
[37] Sen A.K. and Pattanaik P.K., Necessary and sufficient conditions for rational choice under majority decision, Journal of Economic Theory 1 (1969), 178-202.

[38] Sönmez T., Strategy-proofness in many-to-one matching problems, Economic Design 1 (1996), 365-380.

[39] Storcken T., Societies with a priori information and anonymous social welfare functions, Methods of Operations Research 54 (1985), 279-291. 


\section{Valorisation}

Valorisation of knowledge refers to the application of scientific knowledge in practice. It aims at creating societal significance from learning by making this information accessible. Valorisation is not just about 'money', but also about how the exchange of learning and ideas help the society.

Specifically, this Valorisation helps me to think about why I spent the last few years of my life as a $\mathrm{PhD}$ researcher and whether my contributions are helpful to the society. I not only tried to contribute to the society by doing research, but I also contributed to the education of others by teaching in a number of courses. In this section, I try to discuss the societal relevance of the topics discussed in this thesis.

The first part of this thesis broadly studies how 'desirable' matchings can occur in society with couples. In real life, we often encounter scenarios where an institution has to be matched with individuals, be it schools with students, hospitals with doctors, firms with workers, etc. Often, there are individuals who want to be matched to the same location. Thus, it is important to know when it is possible to avoid undesirable matchings. This thesis looks at different conditions which might be necessary to achieve the desired results. 
In the second chapter, we study under what conditions can members of the couple deviate from their individual preferences to be together. This is a very practical problem which couples often face. For example, a person might prefer the school at Amsterdam to the school at Maastricht, but might be willing to change her preference if her better half prefers the school at Maastricht to that at Amsterdam just to be together with her partner.

In the third chapter, we study whether combining individual preferences to get a preference for couples is enough to get a desirable matching. The result we obtain is somewhat counter intuitive, but it is still applicable in society today, since it shows that even if the individuals form a couple, there still needs to be altruism to avoid undesirable matchings. This is because, in the above example, the combination of individual preferences has no preference implication between the two situations where both the agents are matched to the same school. This creates a problem of ambiguity, which can only be overcome if there is a sense of altruism among the members of the couple.

The second part of the thesis deals with the aggregation of individual opinions and preferences to reach a collective decision outcome. This is widely used in many situations, for example in voting, or enacting a law in the constitution, deciding on the location of a public good based on peoples' preferences, etc. In the fourth chapter, we study the situation in which a part of individuals' preferences is known. This is closely associated with what we encounter in daily life. A part of an individual's preference is revealed by religious beliefs, behavior on internet, political views, health conditions, etc. In our study, for different individuals different preference parts may be known. For instance, a vegetarian prefers cheese to any food item containing meat, where a lactose intolerant person would never prefer cheese to any other dish. This study tries to find the socially acceptable outcome in such a situation. 


\section{Curriculum Vitae}

Shashwat Khare was born on July 15, 1992 in Indore, India. He studied Bachelor of Statistics (B.Stat) at the Indian Statistical Institute, Kolkata. In 2013, he received his BSc degree. He studied Master of Statistics (M.Stat) with Quantitative Specialisation from the same institute and graduated in 2015.

Then he joined Maastricht University as a PhD candidate under the supervision of Dr. Hans Peters and Dr. Ton Storcken. The outcomes of his research are presented in this thesis. Shashwat presented his research at various international conferences. 
